\title{
Stringy invariants and toric Artin stacks
}

\author{
Matthew Satriano ${ }^{10}$ and Jeremy Usatine ${ }^{2}$ \\ ${ }^{1}$ Department of Pure Mathematics, University of Waterloo, 200 University Avenue West, Waterloo, Ontario, N2L 3G1, Canada; \\ E-mail:msatriano@uwaterloo.ca. \\ ${ }^{2}$ Department of Mathematics, Brown University, 151 Thayer Street, Providence, RI, 02912, USA; \\ E-mail: jeremy_usatine@brown.edu.
}

Received: 4 October 2021; Revised: 22 December 2021; Accepted: 8 January 2022

2020 Mathematics Subject Classification: Primary - 14E18; Secondary - 14D23, 14M25

\begin{abstract}
We propose a conjectural framework for computing Gorenstein measures and stringy Hodge numbers in terms of motivic integration over arcs of smooth Artin stacks, and we verify this framework in the case of fantastacks, which are certain toric Artin stacks that provide (nonseparated) resolutions of singularities for toric varieties. Specifically, let $\mathcal{X}$ be a smooth Artin stack admitting a good moduli space $\pi: \mathcal{X} \rightarrow X$, and assume that $X$ is a variety with log-terminal singularities, $\pi$ induces an isomorphism over a nonempty open subset of $X$ and the exceptional locus of $\pi$ has codimension at least 2 . We conjecture a change-of-variables formula relating the motivic measure for $\mathcal{X}$ to the Gorenstein measure for $X$ and functions measuring the degree to which $\pi$ is nonseparated. We also conjecture that if the stabilisers of $\mathcal{X}$ are special groups in the sense of Serre, then almost all arcs of $X$ lift to arcs of $\mathcal{X}$, and we explain how in this case (assuming a finiteness hypothesis satisfied by fantastacks) our conjectures imply a formula for the stringy Hodge numbers of $X$ in terms of a certain motivic integral over the arcs of $\mathcal{X}$. We prove these conjectures in the case where $\mathcal{X}$ is a fantastack.
\end{abstract}

\section{Contents}

1 Introduction $\quad 2$

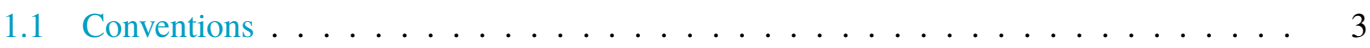

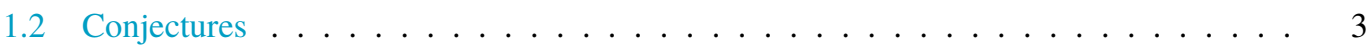

1.3 Main results . . . . . . . . . . . . . . . . . . . . . 5 5

2 Preliminaries $\quad 5$

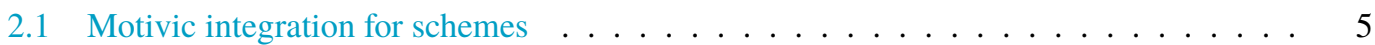

2.2 The Grothendieck ring of stacks and constructible subsets . . . . . . . . . . . . 7

2.3 Toric Artin stacks . . . . . . . . . . . . . . . . . . . 9

3 Motivic integration for stacks $\quad 12$

3.1 Jet schemes of quotient stacks . . . . . . . . . . . . . . . . . . 15

3.2 Truncation morphisms and quotient stacks . . . . . . . . . . . . . . . 16

3.3 Properties of motivic integration for quotient stacks . . . . . . . . . . . . . 18

3.4 Nonseparatedness functions . . . . . . . . . . . . . . . . . . . . . 19

3.5 Motivic integration for smooth stacks via the cotangent complex . . . . . . . . 20

4 Fibres of the map of arcs $\quad 23$

4.1 Tropicalising $\operatorname{arcs}$ of toric stacks $\ldots \ldots \ldots \ldots$

(c) The Author(s), 2022. Published by Cambridge University Press. This is an Open Access article, distributed under the terms of the Creative Commons Attribution licence (https://creativecommons.org/licenses/by/4.0/), which permits unrestricted re-use, distribution, and reproduction in any medium, provided the original work is properly cited. 
4.2 Lifting $\operatorname{arcs}$ to a fantastack . . . . . . . . . . . . . . . . 26

5 Fibres of the maps of jets $\quad 28$

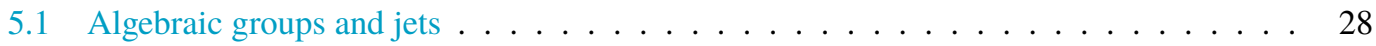

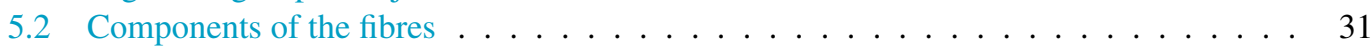

6 Gorenstein measure and toric varieties $\quad 37$

6.1 Gorenstein measure and monomial ideals . . . . . . . . . . . . . . . . . 38

6.2 Gorenstein measure and toric modifications . . . . . . . . . . . . . . . . 40

7 Motivic measure and canonical stacks $\quad \mathbf{4 2}$

7.1 Canonical stacks and preimages of measurable subsets . . . . . . . . . . . . 43

7.2 Quotient by an algebraic torus . . . . . . . . . . . . . . . . . 45

7.3 Canonical stacks and preimages of cocharacters . . . . . . . . . . 45

8 Stringy invariants and toric Artin stacks: Proof of Theorem 1.7 47

9 Fantastacks with special stabilisers: Proof of Theorem 1.11 49

9.1 A combinatorial criterion for special stabilisers . . . . . . . . . . . . . . 49

10 Quotients by $\mathrm{SL}_{2}$ and cones over Grassmannians $\quad 52$

\section{Introduction}

Let $X$ be a variety with log-terminal singularities. Motivated by mirror symmetry for singular CalabiYau varieties, Batyrev introduced stringy Hodge numbers for $X$ in [Bat98], which are defined in terms of a resolution of singularities. In particular, if $X$ admits a crepant resolution $Y \rightarrow X$ by a smooth projective variety $Y$, then the stringy Hodge numbers of $X$ are equal to the usual Hodge numbers of $Y$. In [DL02], Denef and Loeser defined the Gorenstein measure $\mu_{X}^{\text {Gor }}$ on the arc scheme $\mathscr{L}(X)$ of $X$ and used it to prove a McKay correspondence that refines the McKay correspondence conjectured by Reid in [Rei92] and proved by Batyrev in [Bat99]. The measure $\mu_{X}^{\text {Gor }}$ takes values in a modified Grothendieck ring of varieties $\widehat{\mathscr{M}}_{k}\left[\mathbb{L}^{1 / m}\right]$ and is a refinement of the stringy Hodge numbers of $X$. If $X$ admits a crepant resolution $Y \rightarrow X$, then $\mu_{X}^{\mathrm{Gor}}$ is essentially equivalent to the usual motivic measure $\mu_{Y}$ on $\mathscr{L}(Y)$ as introduced by Kontsevich in [Kon95].

A major open question asks whether or not the stringy Hodge numbers of projective varieties are nonnegative, as conjectured by Batyrev in [Bat98, Conjecture 3.10]. A stronger conjecture predicts that stringy Hodge numbers of projective varieties are equal to the dimensions of some kind of cohomology groups. In [Yas04], these conjectures were proved in the case where $X$ has quotient singularites. Yasuda showed that in that case, if $\mathcal{X}$ is the canonical smooth Deligne-Mumford stack over $X$, then the stringy Hodge numbers of $X$ are equal to the orbifold Hodge numbers of $\mathcal{X}$ in the sense of Chen and Ruan [CR04]. To prove this result, Yasuda introduced a notion of motivic integration (further developed in [Yas06, Yas 19]) for Deligne-Mumford stacks and proved a formula expressing $\mu_{X}^{\text {Gor }}$ in terms of certain motivic integrals over arcs of $\mathcal{X}$. When $X$ is projective, those integrals over arcs of $\mathcal{X}$ compute the orbifold Hodge numbers of $\mathcal{X}$.

In this paper, we initiate a similar program for varieties with singularities that are worse than quotient singularities. Such varieties never arise as the coarse space of a smooth Deligne-Mumford stack, so one is instead forced to consider Artin stacks. A major technicality is that such stacks are not separated. This leads us to define new functions $\operatorname{sep}_{\pi, \mathcal{C}}$, discussed later, which measure the degree to which an Artin stack is not separated. These functions play a key role in our theory.

The class of varieties we consider are those $X$ occurring as the good moduli space (in the sense of [Alp13]) of a smooth Artin stack $\mathcal{X}$; varieties of this form arise naturally in the context of geometric invariant theory. We require that the map $\pi: \mathcal{X} \rightarrow X$ induce an isomorphism over a nonempty open subset of $X$ and that the exceptional locus of $\pi$ have codimension at least 2 . In other words, we want $\mathcal{X}$ to be a 'small' resolution of $X$. We conjecture a relationship between $\mu_{X}^{\text {Gor }}$ and a motivic measure $\mu_{\mathcal{X}}$ on the arc stack $\mathscr{L}(\mathcal{X})$ of $\mathcal{X}$. This relationship involves integrating $\operatorname{sep}_{\pi, \mathcal{C}}: \mathscr{L}(X) \rightarrow \mathbb{N}$ against $\mu_{X}^{\text {Gor }}$. This function $\operatorname{sep}_{\pi, \mathcal{C}}$ counts the number of arcs of $\mathcal{X}$ (in some auxiliary measurable subset $\mathcal{C}$ ), up to 
isomorphism, above each arc of $X$, and can therefore be thought of as an invariant which measures the nonseparatedness of $\pi$. We emphasise that this conjectural relationship is not 'built into' our definition of $\mu_{\mathcal{X}}$. In fact, our notion of $\mu_{\mathcal{X}}$ is straightforward: it is more or less Kontsevich's original motivic measure, except that various notions for schemes are replaced with the obvious analogues for Artin stacks. When the stabilisers of $\mathcal{X}$ are special groups in the sense of $\operatorname{Serre}^{1}$ and $\mathscr{L}(\pi): \mathscr{L}(\mathcal{X}) \rightarrow \mathscr{L}(X)$ has finite fibres outside a set of measure 0 , our conjectures imply a formula expressing the stringy Hodge numbers of $X$ in terms of a certain motivic integral over $\mathscr{L}(\mathcal{X})$.

We prove that our conjectures hold when $X$ is a toric variety and $\mathcal{X}$ is a fantastack - that is, a type of smooth toric Artin stack in the sense of [GS15a, GS15b]. Fantastacks are a broad class of toric stacks that allow one to simultaneously have any specified toric variety $X$ as a good moduli space while also obtaining stabilisers with arbitrarily large dimension. An important special case of fantastacks (and their products with algebraic tori) is the so-called canonical stack $\mathcal{X}$ over a toric variety $X$. When $X$ has quotient singularities, $\mathcal{X}$ is the canonical smooth Deligne-Mumford stack over $X$; when $X$ has worse singularities, the good moduli space of $\mathcal{X}$ is still $X$, but $\mathcal{X}$ is an Artin stack that is not Deligne-Mumford.

\subsection{Conventions}

Throughout this paper, $k$ will be an algebraically closed field with characteristic 0 . All Artin stacks will be assumed to have affine (geometric) stabilisers, and all toric varieties will be assumed to be normal. For any stack $\mathcal{X}$ over $k$, we will let $|\mathcal{X}|$ denote the topological space associated to $\mathcal{X}$, and for any $k$-algebra $R$, we will let $\overline{\mathcal{X}}(R)$ denote the set of isomorphism classes of the category $\mathcal{X}(R)$.

\subsection{Conjectures}

Our first conjecture predicts a relationship between $\mu_{X}^{\text {Gor }}$ and $\mu_{\mathcal{X}}$. As already mentioned, our formula involves integrals weighted by functions $\operatorname{sep}_{\pi, \mathcal{C}}$ that measure the degree to which $\pi$ is not separated. We refer the reader to section 3 for precise definitions of the arc stack $\mathscr{L}(\mathcal{X})$ and its motivic measure $\mu_{\mathcal{X}}$, and to subsection 3.4 for the definition of $\operatorname{sep}_{\pi, \mathcal{C}}$ and its integral $\int_{C} \operatorname{sep}_{\pi, \mathcal{C}} \mathrm{d} \mu_{X}^{\mathrm{Gor}}$.

Conjecture 1.1. Let $\mathcal{X}$ be a smooth irreducible Artin stack over $k$ admitting a good moduli space $\pi: \mathcal{X} \rightarrow X$, where $X$ is a separated $k$-scheme and has log-terminal singularities. Assume that $\pi$ induces an isomorphism over a nonempty open subset of $X$, and that the exceptional locus of $\pi$ has codimension at least 2 .

If $\mathcal{C} \subset|\mathscr{L}(\mathcal{X})|$ is a measurable subset such that $\operatorname{sep}_{\pi, \mathcal{C}}: \mathscr{L}(X) \rightarrow \mathbb{N} \cup\{\infty\}$ is finite outside a set of measure 0 , then $\operatorname{sep}_{\pi, \mathcal{C}}: \mathscr{L}(X) \rightarrow \mathbb{N} \cup\{\infty\}$ has measurable fibres, and for any measurable subset $C \subset \mathscr{L}(X)$, the set $\mathcal{C} \cap \mathscr{L}(\pi)^{-1}(C) \subset|\mathscr{L}(\mathcal{X})|$ is measurable and satisfies

$$
\mu_{\mathcal{X}}\left(\mathcal{C} \cap \mathscr{L}(\pi)^{-1}(C)\right)=\int_{C} \operatorname{sep}_{\pi, \mathcal{C}} \mathrm{d} \mu_{X}^{\mathrm{Gor}} \in \widehat{\mathscr{M}}_{k}\left[\mathbb{L}^{1 / m}\right],
$$

where $m \in \mathbb{Z}_{>0}$ is such that $m K_{X}$ is Cartier.

This conjecture predicts that for the purpose of computing $\mu_{X}^{\mathrm{Gor}}$, the stack $\mathcal{X}$ behaves like a crepant resolution of $X$, except we need to correct by $\operatorname{sep}_{\pi, \mathcal{C}}$ to account for the fact that $\mathcal{X}$ is not separated over $X$. Set

$$
\operatorname{sep}_{\pi}=\operatorname{sep}_{\pi,|\mathscr{L}(\mathcal{X})|}
$$

Notice that Conjecture 1.1 implies, in particular, that the motivic measure $\mu_{\mathcal{X}}$ 'does not see' how $\mu_{X}^{\text {Gor }}$ behaves on the set $\operatorname{sep}_{\pi}^{-1}(0) \subset \mathscr{L}(X)$. This set can have nonzero measure because $\pi: \mathcal{X} \rightarrow X$ does not necessarily satisfy the 'strict valuative criterion' - that is, there may exist arcs of $X$ (even outside a set

${ }^{1} G$ is special if every $G$-torsor is Zariski locally trivial. 
of measure 0) that do not lift to arcs of $\mathcal{X}$. Thus in general we cannot use this conjecture to compute the total Gorenstein measure $\mu_{X}^{\mathrm{Gor}}(\mathscr{L}(X))$, which specialises to the stringy Hodge numbers of $X$. This issue already occurs in the case where $\mathcal{X}$ is a Deligne-Mumford stack. For this reason, Yasuda uses a notion of 'twisted arcs' of $\mathcal{X}$ instead of usual arcs of $\mathcal{X}$, and this is why the inertia of $\mathcal{X}$ and orbifold Hodge numbers appear in Yasuda's setting. We take a different approach, emphasising a setting in which the next conjecture predicts that almost all arcs of $X$ lift to $\operatorname{arcs}$ of $\mathcal{X}$.

Conjecture 1.2. Let $\mathcal{X}$ be a finite-type Artin stack over $k$ admitting a good moduli space $\pi: \mathcal{X} \rightarrow X$. Assume that $X$ is an irreducible $k$-scheme and that $\pi$ induces an isomorphism over a nonempty open subset of $X$. If the stabilisers of $\mathcal{X}$ are all special groups, then $\operatorname{sep}_{\pi}^{-1}(0) \subset \mathscr{L}(X)$ is measurable and

$$
\mu_{X}\left(\operatorname{sep}_{\pi}^{-1}(0)\right)=0
$$

where we note that $\mu_{X}$ is the usual (non-Gorenstein) motivic measure on $\mathscr{L}(X)$.

Remark 1.3. All special groups are connected, so if $\mathcal{X}$ is a Deligne-Mumford stack whose stabilisers are special groups, then its stabilisers are all trivial. Thus Conjecture 1.2 highlights a setting that is 'orthogonal' to the setting considered by Yasuda.

Our next question is motivated by the fact that if $\operatorname{sep}_{\pi}$ is finite outside a set of measure 0 , we may then consider the special case of Conjecture 1.1 where $\mathcal{C}=|\mathscr{L}(\mathcal{X})|$.

Question 1.4. Let $\mathcal{X}$ be a finite-type Artin stack over $k$ admitting a good moduli space $\pi: \mathcal{X} \rightarrow X$. Assume that $X$ is an irreducible $k$-scheme and that $\pi$ induces an isomorphism over a nonempty open subset of $X$. When is

$$
\mu_{X}\left(\operatorname{sep}_{\pi}^{-1}(\infty)\right)=0
$$

satisfied?

We now give an application of this framework to computing stringy Hodge numbers. In subsection 3.4, we introduce the function $\operatorname{sep}_{\mathcal{X}}=1 /\left(\operatorname{sep}_{\pi} \circ \mathscr{L}(\pi)\right):|\mathscr{L}(\mathcal{X})| \rightarrow \mathbb{Q}_{\geq 0} \cup\{\infty\}$. We think of its integral $\int_{\mathscr{L}(\mathcal{X})} \operatorname{sep}_{\mathcal{X}} \mathrm{d} \mu_{\mathcal{X}}$ as a kind of motivic class of $\mathscr{L}(\mathcal{X})$ corrected by $\operatorname{sep}_{\mathcal{X}}$ to account for the fact that $\mathcal{X}$ is not separated. We refer the reader to subsection 3.4 for the precise definitions of $\int_{\mathscr{L}(\mathcal{X})} \operatorname{sep} \mathcal{X} \mathrm{d} \mu_{\mathcal{X}}$ and the ring $\overline{\mathscr{M}_{k} \otimes_{\mathbb{Z}} \mathbb{Q}}$. The next proposition is then immediate:

Proposition 1.5. With hypotheses as in Conjecture 1.1, if the stablisers of $\mathcal{X}$ are special groups and $\mu_{X}\left(\operatorname{sep}_{\pi}^{-1}(\infty)\right)=0$, then Conjecture 1.1 and Conjecture 1.2 imply that the fibres of $\operatorname{sep}_{\mathcal{X}}:|\mathscr{L}(\mathcal{X})| \rightarrow$ $\mathbb{Q}_{\geq 0}$ are measurable and

$$
\mu_{X}^{\mathrm{Gor}}(\mathscr{L}(X))=\int_{\mathscr{L}(\mathcal{X})} \operatorname{sep}_{\mathcal{X}} \mathrm{d} \mu_{\mathcal{X}} \in \overline{\mathscr{M}_{k} \otimes_{\mathbb{Z}} \mathbb{Q}}
$$

Since the stringy Hodge-Deligne invariant of $X$ is a specialisation of the image of $\mu_{X}^{\text {Gor }}(\mathscr{L}(X))$ in $\left(\overline{M_{k} \otimes_{\mathbb{Z}} \mathbb{Q}}\right)\left[\mathbb{L}^{1 / m}\right] \supset \overline{M_{k} \otimes_{\mathbb{Z}} \mathbb{Q}}$, Proposition 1.5 provides a conjectural formula for the stringy Hodge numbers of $X$ (when the stringy Hodge numbers exist - that is, when the stringy Hodge-Deligne invariant is a polynomial).

We envision a few potential applications of this framework. Noting that the good moduli space map $\pi: \mathcal{X} \rightarrow X$ is intrinsic to the stack $\mathcal{X}$ and therefore so is the integral $\int_{\mathscr{L}(\mathcal{X})} \operatorname{sep}_{\mathcal{X}} \mathrm{d} \mu_{\mathcal{X}}$, we hope that a cohomological interpretation of $\int_{\mathscr{L}(\mathcal{X})} \operatorname{sep} \mathrm{p}_{\mathcal{X}} \mathrm{d} \mu_{\mathcal{X}}$ will lead to progress on Batyrev's conjecture on the nonnegativity of stringy Hodge numbers. We also hope that by considering Proposition 1.5 as a kind of McKay correspondence, our conjectures will lead to new representation-theoretic statements for positive-dimensional algebraic groups. 
Remark 1.6. The hypothesis $\mu_{X}\left(\operatorname{sep}_{\pi}^{-1}(\infty)\right)=0$ in Proposition 1.5 allows us to make a canonical choice for $\mathcal{C}$ in Conjecture 1.1, specifically the choice $\mathcal{C}=|\mathscr{L}(\mathcal{X})|$. We hope that even when this hypothesis does not hold, one can still (after an appropriate generalisation of the notion of an arc) make a canonical choice for $\mathcal{C}$. This is a subject of our ongoing research.

\subsection{Main results}

Our first main result is that Conjecture 1.1 holds, and $\mu_{X}\left(\operatorname{sep}_{\pi}^{-1}(\infty)\right)=0$, when $\mathcal{X}$ is a fantastack and $\mathcal{C}=|\mathscr{L}(\mathcal{X})|$. In particular, our framework applies to the Gorenstein measure of any toric variety $X$ with log-terminal singularities.

Theorem 1.7. Conjecture 1.1 holds and $\mu_{X}\left(\operatorname{sep}_{\pi}^{-1}(\infty)\right)=0$ when $\mathcal{X}$ is a fantastack and $\mathcal{C}=|\mathscr{L}(\mathcal{X})|$.

Remark 1.8. In fact, our techniques prove a more general result: the conclusions of Conjecture 1.1 hold when $\mathcal{C}=|\mathscr{L}(\mathcal{X})|$ and $\mathcal{X}$ is a fantastack satisfying a certain combinatorial condition analogous to $\mathcal{X} \rightarrow X$ being 'crepant' (see Remark 2.20 for more details). It is important to note here that unlike the case of Deligne-Mumford stacks, defining $K_{\mathcal{X}}$ for Artin stacks is a subtle issue, so there is no a priori obvious definition one can take for $\mathcal{X} \rightarrow X$ to be crepant.

Remark 1.9. We note that the stacks $\mathcal{X}$ in Theorem 1.7 all have commutative stabilisers. In order to provide evidence that Conjecture 1.1 should not be limited to the setting of commutative stabilisers, we also verify that it holds in examples that involve $\mathrm{SL}_{2}$ as a stabiliser (see section 10). These examples also demonstrate the flexibility in choosing the auxiliary set $\mathcal{C} \subset|\mathscr{L}(\mathcal{X})|$.

Remark 1.10. Theorem 1.7 can be thought of as a motivic change-of-variables formula. We note that Balwe introduced versions of motivic integration for Artin $n$-stacks [Bal08, Bal15] and proved a changeof-variables formula [Bal08, Theorem 7.2.5]. However, Theorem 1.7 cannot be obtained from Balwe's result, as the map $\pi: \mathcal{X} \rightarrow X$ does not satisfy Balwe's hypotheses: specifically, $\pi$ is not '0-truncated'.

The three main steps of proving Theorem 1.7 are as follows. First, we give a combinatorial description of the fibres of the map $\mathscr{L}(\pi): \mathscr{L}(\mathcal{X}) \rightarrow \mathscr{L}(X)$. Second, we show that for sufficiently large $n$, the map of jets $\mathscr{L}_{n}(\pi): \mathscr{L}_{n}(\mathcal{X}) \rightarrow \mathscr{L}_{n}(X)$ has constant fibres (after taking the fibres' reduced structure) over certain combinatorially defined pieces of $\mathscr{L}_{n}(X)$. These two steps allow us to reduce Theorem 1.7 to the final step: verifying the case where the measurable sets $C$ are certain combinatorially defined subsets of $\mathscr{L}(X)$. A key ingredient in this final step is Theorem 3.9 and its corollary, Corollary 3.16, which show how to compute the motivic measure of the stack quotient of a variety by the action of a special group.

Our second main result is that Conjecture 1.2 holds for fantastacks.

Theorem 1.11. Conjecture 1.2 holds when $\mathcal{X}$ is a fantastack.

An essential ingredient in proving Theorem 1.11 is Theorem 9.1, which may be of independent interest, as it provides a combinatorial criterion to check whether or not the stabilisers of a fantastack are special groups.

\section{Preliminaries}

In this section, we introduce notation and recall some facts about motivic integration for schemes and the Gorenstein measure, the Grothendieck ring of stacks and constructible subsets and toric Artin stacks.

\subsection{Motivic integration for schemes}

If $X$ is a $k$-scheme, for each $n \in \mathbb{N}$ we will let $\mathscr{L}_{n}(X)$ denote the $n$th jet scheme of $X$; for each $n \geq m$ we will let $\theta_{m}^{n}: \mathscr{L}_{n}(X) \rightarrow \mathscr{L}_{m}(X)$ denote the truncation morphism; we will let $\mathscr{L}(X)=\lim _{n} \mathscr{L}_{n}(X)$ denote the arc scheme of $X$; and for each $n \in \mathbb{N}$ we will let $\theta_{n}: \mathscr{L}(X) \rightarrow \mathscr{L}_{n}(X)$ denote the canonical 
morphism, which is also referred to as a truncation morphism. For any $k$-algebra $R$ and $k$-scheme $X$, the map $X(R \llbracket t \rrbracket) \rightarrow \mathscr{L}(X)(R)$ is bijective by [Bha16, Theorem 1.1], and we will often implicitly make this identification.

We will let $K_{0}\left(\operatorname{Var}_{k}\right)$ denote the Grothendieck ring of finite type $k$-schemes; for each finite-type $k$-scheme $X$ we will let $\mathrm{e}(X) \in K_{0}\left(\operatorname{Var}_{k}\right)$ denote its class; we will let $\mathbb{L}=\mathrm{e}\left(\mathbb{A}_{k}^{1}\right) \in K_{0}\left(\operatorname{Var}_{k}\right)$ denote the class of the affine line; and for each constructible subset $C$ of a finite-type $k$-scheme we will let $\mathrm{e}(C) \in K_{0}\left(\operatorname{Var}_{k}\right)$ denote its class.

We will let $\mathscr{M}_{k}$ denote the ring obtained by inverting $\mathbb{L}$ in $K_{0}\left(\operatorname{Var}_{k}\right)$. For each $\Theta \in \mathscr{M}_{k}$, let $\operatorname{dim}(\Theta) \in \mathbb{Z} \cup\{-\infty\}$ denote the infimum over all $d \in \mathbb{Z}$ such that $\Theta$ is in the subgroup of $\mathscr{M}_{k}$ generated by elements of the form $\mathrm{e}(X) \mathbb{L}^{-n}$ with $\operatorname{dim}(X)-n \leq d$, and let $\|\Theta\|=\exp (\operatorname{dim}(\Theta))$. We will let $\widehat{\mathscr{M}}_{k}$ denote the separated completion of $\mathscr{M}_{k}$ with respect to the non-Archimedean seminorm $\|\cdot\|$, and we will also let $\|\cdot\|$ denote the non-Archimedean norm on $\widehat{\mathscr{M}}_{k}$. For any $m \in \mathbb{Z}_{>0}$, we will let $\widehat{M}_{k}\left[\mathbb{L}^{1 / m}\right]=\widehat{\mathscr{M}}_{k}[t] /\left(t^{m}-\mathbb{L}\right)$, we will let $\mathbb{L}^{1 / m}$ denote the image of $t$ in $\widehat{\mathscr{M}}_{k}\left[\mathbb{L}^{1 / m}\right]$ and we will endow $\widehat{\mathscr{M}}_{k}\left[\mathbb{L}^{1 / m}\right]$ with the topology induced by the equality

$$
\widehat{\mathscr{M}}_{k}\left[\mathbb{L}^{1 / m}\right]=\bigoplus_{\ell=0}^{m-1} \widehat{\mathscr{M}}_{k} \cdot\left(\mathbb{L}^{1 / m}\right)^{\ell},
$$

where each summand $\widehat{\mathscr{M}}_{k} \cdot\left(\mathbb{L}^{1 / m}\right)^{\ell}$ has the topology induced by the bijection

$$
\widehat{\mathscr{M}}_{k} \rightarrow \widehat{\mathscr{M}}_{k} \cdot\left(\mathbb{L}^{1 / m}\right)^{\ell}: \Theta \mapsto \Theta \cdot\left(\mathbb{L}^{1 / m}\right)^{\ell}
$$

We note that in the foregoing and throughout this paper, if $\Theta$ is an element of $K_{0}\left(\operatorname{Var}_{k}\right), \mathscr{M}_{k}$ or $\widehat{\mathscr{M}}_{k}$, we slightly abuse notation by also using $\Theta$ to refer to its image under any of the ring maps $K_{0}\left(\operatorname{Var}_{k}\right) \rightarrow \mathscr{M}_{k} \rightarrow \widehat{\mathscr{M}}_{k} \rightarrow \widehat{\mathscr{M}}_{k}\left[\mathbb{L}^{1 / m}\right]$

If $X$ is an equidimensional finite-type $k$-scheme and $C \subset \mathscr{L}(X)$ is a cylinder - that is, $C=\left(\theta_{n}\right)^{-1}\left(C_{n}\right)$ for some $n \in \mathbb{N}$ and some constructible subset $C_{n} \subset \mathscr{L}_{n}(X)$ - we will let $\mu_{X}(C) \in \widehat{\mathscr{M}}_{k}$ denote the motivic measure of $C$, so by definition

$$
\mu_{X}(C)=\lim _{n \rightarrow \infty} \mathrm{e}\left(\theta_{n}(C)\right) \mathbb{L}^{-(n+1) \operatorname{dim} X} \in \widehat{\mathscr{M}}_{k},
$$

where we note that each $\theta_{n}(C)$ is constructible (for example, by [CLNS18, Chapter 5, Corollary 1.5.7(b)]) and this limit exists (for example, by [CLNS18, Chapter 6, Theorem 2.5.1]). The motivic measure $\mu_{X}$ can be extended to the class of so-called measurable subsets of $\mathscr{L}(X)$, whose definition we now recall.

Definition 2.1. Let $X$ be an equidimensional finite-type scheme over $k$, set $C \subset \mathscr{L}(X)$ and $\varepsilon \in \mathbb{R}_{>0}$, let $I$ be a set, let $C^{(0)} \subset \mathscr{L}(X)$ be a cylinder and let $\left\{C^{(i)}\right\}_{i \in I}$ be a collection of cylinders in $\mathscr{L}(X)$.

The data $\left(C^{(0)},\left(C^{i}\right)_{i \in I}\right)$ is called a cylindrical $\varepsilon$-approximation of $C$ if

$$
\left(C \cup C^{(0)}\right) \backslash\left(C \cap C^{(0)}\right) \subset \bigcup_{i \in I} C^{(i)}
$$

and, for all $i \in I$,

$$
\left\|\mu_{X}\left(C^{(i)}\right)\right\|<\varepsilon
$$

Definition 2.2. Let $X$ be an equidimensional finite-type scheme over $k$, and set $C \subset \mathscr{L}(X)$. The set $C$ is called measurable if for any $\varepsilon \in \mathbb{R}_{>0}$ there exists a cylindrical $\varepsilon$-approximation of $C$. 
The motivic measure of a measurable subset $C \subset \mathscr{L}(X)$ is defined to be the unique element $\mu_{X}(C) \in \widehat{\mathscr{M}}_{k}$ such that for any $\varepsilon \in \mathbb{R}_{>0}$ and any cylindrical $\varepsilon$-approximation $\left(C^{(0)},\left(C^{(i)}\right)_{i \in I}\right)$ of $C$, we have

$$
\left\|\mu_{X}(C)-\mu_{X}\left(C^{(0)}\right)\right\|<\varepsilon .
$$

Such an element $\mu_{X}(C)$ exists by [CLNS18, Chapter 6, Theorem 3.3.2].

For the remainder of this subsection, let $X$ be an integral finite-type separated $k$-scheme with logterminal singularities. We will set notation relevant for the Gorenstein measure associated to $X$. We will let $K_{X}$ denote the canonical divisor on $X$. If $m \in \mathbb{Z}_{>0}$ is such that $m K_{X}$ is Cartier, we will let $\omega_{X, m}=\iota_{*}\left(\left(\Omega_{X_{\mathrm{sm}}}^{\operatorname{dim}}\right)^{\otimes m}\right)$, where $\iota: X_{\mathrm{sm}} \hookrightarrow X$ is the inclusion of the smooth locus of $X$, and we will let $\mathscr{J}_{X, m}$ denote the unique ideal sheaf on $X$ such that the image of $\left(\Omega_{X}^{\operatorname{dim} X}\right)^{\otimes m} \rightarrow \omega_{X, m}$ is equal to $\mathscr{I}_{X, m} \omega_{X, m}$. If $C \subset \mathscr{L}(X)$ is measurable, we will let $\mu_{X}^{\text {Gor }}(C)$ denote the Gorenstein measure of $C$, so by definition,

$$
\begin{aligned}
\mu_{X}^{\text {Gor }}(C) & =\int_{C}\left(\mathbb{L}^{1 / m}\right)^{\text {ord } \mathcal{F}_{X, m}} \mathrm{~d} \mu_{X} \\
& =\sum_{n=0}^{\infty}\left(\mathbb{L}^{1 / m}\right)^{n} \mu_{X}\left(\operatorname{ord}_{\mathcal{F}_{X, m}}^{-1}(n) \cap C\right) \in \widehat{\mathscr{M}}_{k}\left[\mathbb{L}^{1 / m}\right]
\end{aligned}
$$

where $m \in \mathbb{Z}_{>0}$ is such that $m K_{X}$ is Cartier and ord $\mathcal{F}_{X, m}: \mathscr{L}(X) \rightarrow \mathbb{N} \cup\{\infty\}$ is the order function of the ideal sheaf $\mathscr{J}_{X, m}$. The following proposition is easy to check using the definition of $\mu_{X}^{\text {Gor }}$ and standard properties of $\mu_{X}$ given in [CLNS18, Chapter 6, Proposition 3.4.3]:

Proposition 2.3. Let $\left\{C^{(i)}\right\}_{i \in \mathbb{N}}$ be a sequence of pairwise disjoint measurable subsets of $\mathscr{L}(X)$ such that $C=\bigcup_{i=0}^{\infty} C^{(i)}$ is measurable. Then

$$
\lim _{i \rightarrow \infty} \mu_{X}^{\mathrm{Gor}}\left(C^{(i)}\right)=0
$$

and

$$
\mu_{X}^{\mathrm{Gor}}(C)=\sum_{i=0}^{\infty} \mu_{X}^{\mathrm{Gor}}\left(C^{(i)}\right)
$$

\subsection{The Grothendieck ring of stacks and constructible subsets}

We will let $K_{0}\left(\mathbf{S t a c k}_{k}\right)$ denote the Grothendieck ring of stacks in the sense of [Eke09], and for each finite-type Artin stack $\mathcal{X}$ over $k$, we will let e $(\mathcal{X}) \in K_{0}\left(\mathbf{S t a c k}_{k}\right)$ denote the class of $\mathcal{X}$. If $K_{0}\left(\operatorname{Var}_{k}\right)\left[\mathbb{L}^{-1},\left\{\left(\mathbb{L}^{n}-1\right)^{-1}\right\}_{n \in \mathbb{Z}_{>0}}\right]$ is the ring obtained from $K_{0}\left(\operatorname{Var}_{k}\right)$ by inverting $\mathbb{L}$ and $\left(\mathbb{L}^{n}-1\right)$ for all $n \in \mathbb{Z}_{>0}$, then the obvious ring map $K_{0}\left(\operatorname{Var}_{k}\right) \rightarrow K_{0}\left(\mathbf{S t a c k}_{k}\right)$ induces an isomorphism

$$
K_{0}\left(\operatorname{Var}_{k}\right)\left[\mathbb{L}^{-1},\left\{\left(\mathbb{L}^{n}-1\right)^{-1}\right\}_{n \in \mathbb{Z}_{>0}}\right] \cong K_{0}\left(\operatorname{Stack}_{k}\right),
$$

by [Eke09, Theorem 1.2]. Therefore there exists a unique ring map

$$
K_{0}\left(\operatorname{Stack}_{k}\right) \rightarrow \widehat{\mathscr{M}}_{k},
$$

whose composition with $K_{0}\left(\operatorname{Var}_{k}\right) \rightarrow K_{0}\left(\mathbf{S t a c k}_{k}\right)$ is the usual map $K_{0}\left(\operatorname{Var}_{k}\right) \rightarrow \widehat{M}_{k}$. If $\Theta \in K_{0}$ $\left(\mathbf{S t a c k}_{k}\right)$, we will slightly abuse notation by also using $\Theta$ to refer to its image under $K_{0}\left(\mathbf{S t a c k}_{k}\right) \rightarrow \widehat{\mathscr{M}}_{k}$. 
By [Eke09, Propositions 1.1(iii) and 1.4(i)], if $G$ is a special group over $k$, then e $(G) \in K_{0}\left(\mathbf{S t a c k}_{k}\right)$ is a unit, and for any finite-type $k$-scheme $X$ with $G$-action, the class of the stack quotient is

$$
\mathrm{e}([X / G])=\mathrm{e}(X) \mathrm{e}(G)^{-1} \in K_{0}\left(\operatorname{Stack}_{k}\right) .
$$

Remark 2.4. Let $G$ be an algebraic group over $k$. For each $n \in \mathbb{N}$, we give $\mathscr{L}_{n}(G)$ the group structure induced by applying the functor $\mathscr{L}_{n}$ to the group law $G \times_{k} G \rightarrow G$. It is easy to verify that for each $n \in \mathbb{N}$, we have a short exact sequence

$$
1 \rightarrow \mathfrak{g} \rightarrow \mathscr{L}_{n+1}(G) \stackrel{\theta_{n}^{n+1}}{\longrightarrow} \mathscr{L}_{n}(G) \rightarrow 1,
$$

where $\mathfrak{g}$ is the Lie algebra of $G$. Thus by induction on $n$, the fact that $\mathbb{G}_{a}$ is special, the fact that extensions of special groups are special and the fact that $\mathscr{L}_{0}(G) \cong G$, we see that if $G$ is a special group, then each jet scheme $\mathscr{L}_{n}(G)$ is a special group.

To state the next result, we recall that if $\mathcal{X}$ is a finite-type Artin stack over $k$, then the topological space $|\mathcal{X}|$ is Noetherian, so its constructible subsets are precisely those subsets that can be written as a finite union of locally closed subsets.

Proposition 2.5. Let $\mathcal{X}$ be a finite-type Artin stack over $k$ and let $\mathcal{C} \subset|\mathcal{X}|$ be a constructible subset. Then there exists a unique $\mathrm{e}(\mathcal{C}) \in K_{0}\left(\mathbf{S t a c k}_{k}\right)$ that satisfies the following property. If $\left\{\mathcal{X}_{i}\right\}_{i \in I}$ is a finite collection of locally closed substacks $\mathcal{X}_{i}$ of $\mathcal{X}$ such that $\mathcal{C}$ is equal to the disjoint union of the $\left|\mathcal{X}_{i}\right|$, then

$$
\mathrm{e}(\mathcal{C})=\sum_{i \in I} \mathrm{e}\left(\mathcal{X}_{i}\right) \in K_{0}\left(\operatorname{Stack}_{k}\right)
$$

Proof. The proposition holds by the exact same proof used for the analogous statement for schemes in [CLNS18, Chapter 2, Corollary 1.3.5].

If $\mathcal{X}$ is a finite-type Artin stack and $\mathcal{C} \subset|\mathcal{X}|$ is a constructible subset, we will let e $(\mathcal{C})$ denote the class of $\mathcal{C}-$ that is, $\mathrm{e}(\mathcal{C})$ is as in the statement of Proposition 2.5.

We end this subsection with a useful tool to compute the class of a stack.

Definition 2.6. Let $S$ be a scheme, let $Z$ be scheme over $S$, let $\mathcal{Y}$ and $\mathcal{F}$ be Artin stacks over $S$ and let $\xi: \mathcal{Y} \rightarrow Z$ be a morphism over $S$. We say $\xi$ is a piecewise trivial fibration with fibre $\mathcal{F}$ if there exists a finite cover $\left\{Z_{i}\right\}_{i \in I}$ of $Z$ consisting of pairwise disjoint locally closed subschemes $Z_{i} \subset Z$ such that for all $i \in I$,

$$
\left(\mathcal{Y} \times_{Z} Z_{i}\right)_{\mathrm{red}} \cong\left(\mathcal{F} \times_{S} Z_{i}\right)_{\mathrm{red}}
$$

as stacks over $\left(Z_{i}\right)_{\text {red. }}$

Remark 2.7. Let $Z$ be a finite-type scheme over $k$, let $\mathcal{Y}$ and $\mathcal{F}$ be finite-type Artin stacks over $k$ and let $\xi: \mathcal{Y} \rightarrow Z$ be a piecewise trivial fibration with fibre $\mathcal{F}$. Then by Proposition 2.5,

$$
\mathrm{e}(\mathcal{Y})=\mathrm{e}(\mathcal{F}) \mathrm{e}(Z) \in K_{0}\left(\operatorname{Stack}_{k}\right)
$$

The next proposition is well known in the case where $\mathcal{Y}$ is a scheme.

Proposition 2.8. Let $S$ be a Noetherian scheme, let $Z$ be a finite-type scheme over $S$, let $\mathcal{Y}$ and $\mathcal{F}$ be finite-type Artin stacks over $S$ and let $\xi: \mathcal{Y} \rightarrow Z$ be a morphism over $S$. Then $\xi$ is a piecewise trivial fibration with fibre $\mathcal{F}$ if and only if for all $z \in Z$, there exists an isomorphism

$$
\left(\mathcal{Y} \times_{Z} \operatorname{Spec}(k(z))\right)_{\mathrm{red}} \cong\left(\mathcal{F} \times_{S} \operatorname{Spec}(k(z))\right)_{\text {red }}
$$

of stacks over $k(z)$, where $k(z)$ denotes the residue field of $z$. 
Proof. If $\xi$ is a piecewise trivial fibration with fibre $\mathcal{F}$, then for every $z \in Z$, there is a locally closed subset $Z^{\prime} \subseteq Z$ containing $z$ for which $\left(\mathcal{Y} \times_{Z} Z^{\prime}\right)_{\text {red }} \cong\left(\mathcal{F} \times_{S} Z^{\prime}\right)_{\text {red }}$ as $Z_{\text {red }}^{\prime}$-stacks. Then

$$
\begin{aligned}
\left(\mathcal{Y} \times_{Z} \operatorname{Spec} k(z)\right)_{\mathrm{red}} & =\left(\left(\mathcal{Y} \times_{Z} Z^{\prime}\right)_{\mathrm{red}} \times_{Z_{\mathrm{red}}^{\prime}} \operatorname{Spec} k(z)\right)_{\mathrm{red}} \\
& \cong\left(\left(\mathcal{F} \times_{S} Z^{\prime}\right)_{\mathrm{red}} \times_{Z_{\mathrm{red}}^{\prime}} \operatorname{Spec} k(z)\right)_{\mathrm{red}}=\left(\mathcal{F} \times_{S} \operatorname{Spec} k(z)\right)_{\mathrm{red}} .
\end{aligned}
$$

We now show that the converse holds. Since

$$
\left(\mathcal{Y}_{\text {red }} \times_{Z} \operatorname{Spec} k(z)\right)_{\text {red }}=\left(\mathcal{Y} \times_{Z} \operatorname{Spec} k(z)\right)_{\text {red }}
$$

for every $z \in Z$, we can assume $\mathcal{Y}$ is reduced. By Noetherian induction on $Z$, we need only find a nonempty open subset $U \subseteq Z$ for which $\left(\mathcal{Y} \times_{Z} U\right)_{\text {red }} \cong\left(\mathcal{F} \times_{S} U\right)_{\text {red. }}$. Let $z \in Z$ be the generic point of an irreducible component of $Z$; replacing $Z$ by an open affine neighbourhood of $z$, we may further assume $Z$ is affine. Since $\mathcal{O}_{Z, z}$ is a field, $\mathcal{Y} \times_{Z} \operatorname{Spec} k(z)$ is reduced and we hence have a surjective closed immersion

$$
\iota: \mathcal{Y} \times_{Z} \operatorname{Spec} k(z) \cong\left(\mathcal{F} \times{ }_{S} \operatorname{Spec} k(z)\right)_{\text {red }} \rightarrow \mathcal{F} \times{ }_{S} \operatorname{Spec} k(z) .
$$

Now, $\operatorname{Spec} \mathcal{O}_{Z, z}=\lim _{\lambda} U_{\lambda}$ is the inverse limit of open affine neighbourhoods $U_{\lambda} \subseteq Z$ of $z$. Since $Z$ is affine, each map $U_{\lambda} \rightarrow Z$ is affine. Note also that $\mathcal{Y}$ is Noetherian, hence quasicompact and quasiseparated, and that $\mathcal{F} \times{ }_{S} \operatorname{Spec} k(z) \rightarrow \operatorname{Spec} k(z)$ is locally of finite presentation. [Ryd15, Proposition B.2] then shows there is some index $\lambda$ and a morphism $\iota_{\lambda}: \mathcal{Y} \times_{Z} U_{\lambda} \rightarrow \mathcal{F} \times_{S} U_{\lambda}$ whose base change to $\operatorname{Spec} \mathcal{O}_{Z, z}$ is $\iota$. Furthermore, since $\mathcal{F} \times_{S} \operatorname{Spec} k(z) \rightarrow \operatorname{Spec} k(z)$ and $\xi$ are both of finite presentation, [Ryd15, Proposition B.3] shows that after replacing $\lambda$ by a larger index if necessary, we can assume $\iota_{\lambda}$ is a surjective closed immersion, and hence defines an isomorphism $\left(\mathcal{Y} \times_{Z} U_{\lambda}\right)_{\text {red }} \cong\left(\mathcal{F} \times_{S} U_{\lambda}\right)_{\text {red }}$.

\subsection{Toric Artin stacks}

In this subsection, we briefly review the theory of toric stacks introduced in [GS15a], as well as establish some notation. Since the focus in our paper is on the toric variety $X$, and the toric stack $\mathcal{X}$ is viewed as a stacky resolution of $X$, we introduce some notational changes to emphasise this focus.

Definition 2.9. A stacky fan is a pair $(\widetilde{\Sigma}, v)$, where $\widetilde{\Sigma}$ is a fan on a lattice $\widetilde{N}$ and $v: \widetilde{N} \rightarrow N$ is a homomorphism to a lattice $N$ so that the $\operatorname{cokernel} \operatorname{cok} v$ is finite.

A stacky fan $(\widetilde{\Sigma}, v)$ gives rise to a toric stack as follows. Let $X_{\widetilde{\Sigma}}$ be the toric variety associated to $\widetilde{\Sigma}$. Since cok $v$ is finite, $v^{*}$ is injective, so we obtain a surjective homomorphism of tori

$$
\widetilde{T}:=\operatorname{Spec} k\left[\widetilde{N}^{*}\right] \longrightarrow \operatorname{Spec} k\left[N^{*}\right]=: T .
$$

Let $G_{v}$ denote the kernel of this map. Since $\widetilde{T}$ is the torus of $X_{\widetilde{\Sigma}}$, we obtain a $G_{v}$-action on $X_{\widetilde{\Sigma}}$ via the inclusion $G_{v} \subset \widetilde{T}$.

Definition 2.10. With notation as in the previous paragraph, if $(\widetilde{\Sigma}, v)$ is a stacky fan, the associated toric stack is defined to be

$$
\mathcal{X}_{\widetilde{\Sigma}, v}:=\left[X_{\widetilde{\Sigma}} / G_{v}\right]
$$

When $\widetilde{\Sigma}$ is the fan generated by the faces of a single cone $\widetilde{\sigma}$, we denote $\mathcal{X}_{\widetilde{\Sigma}, v}$ by $\mathcal{X}_{\widetilde{\sigma}, v}$.

Example 2.11. If $\Sigma$ is a fan on a lattice $N$ and we let $v$ be the identity map, then $\mathcal{X}_{\Sigma, v}=X_{\Sigma}$. Thus, every toric variety is an example of a toric stack. 
In this paper, we concentrate in particular on fantastacks introduced in [GS15a, Section 4]. These play a particularly important role for us because they allow us to start with a toric variety $X_{\Sigma}$ and produce a smooth stack $\mathcal{X}$ with arbitrary degree of stackyness while maintaining the property that $X$ is the good moduli space of $\mathcal{X}$. In the following, we let $e_{1}, \ldots, e_{r}$ be the standard basis for $\mathbb{Z}^{r}$.

Definition 2.12. Let $\Sigma$ be a fan on a lattice $N$, and let $v: \mathbb{Z}^{r} \rightarrow N$ be a homomorphism with finite cokernel so that every ray of $\Sigma$ contains some $v_{i}:=v\left(e_{i}\right)$ and every $v_{i}$ lies in the support of $\Sigma$. For a cone $\sigma \in \Sigma$, let $\widetilde{\sigma}=\operatorname{cone}\left(\left\{e_{i} \mid v_{i} \in \sigma\right\}\right)$. We define the fan $\widetilde{\Sigma}$ on $\mathbb{Z}^{r}$ as the fan generated by all the $\widetilde{\sigma}$. We define

$$
\mathcal{F}_{\Sigma, v}:=\mathcal{X}_{\widetilde{\Sigma}, v}
$$

Any toric stack isomorphic to some $\mathcal{F}_{\Sigma, v}$ is called a fantastack. When $\Sigma$ is the fan generated by the faces of a cone $\sigma$, we denote $\mathcal{F}_{\Sigma, v}$ by $\mathcal{F}_{\sigma, v}$.

Remark 2.13. By [GS15a, Example 6.24] (compare [Sat13, Theorem 5.5]), the natural map

$$
\mathcal{F}_{\Sigma, v} \longrightarrow X_{\Sigma}
$$

is a good moduli space morphism. Furthermore, fantastacks have moduli interpretations in terms of line bundles and sections, analogous to the moduli interpretation for $\mathbb{P}^{n}$ [GS15a, Section 7].

The next two results will be useful later on.

Proposition 2.14. Let $\sigma$ be a pointed full-dimensional cone and suppose that the good moduli space map $\pi: \mathcal{F}_{\sigma, v} \rightarrow X_{\sigma}$ is an isomorphism over the torus $T$ of $X_{\sigma}$. Then for any $f \in F:=\widetilde{\sigma}^{\vee} \cap \widetilde{N}^{*}$, there exists some $f^{\prime} \in F$ such that

$$
f+f^{\prime} \in P:=\sigma^{\vee} \cap N^{*}
$$

In particular, if $\psi: F \rightarrow \mathbb{N} \cup\{\infty\}$ is a morphism of monoids and $\psi(P) \subset \mathbb{N}$, then $\psi(F) \subset \mathbb{N}$.

Proof. Let $v_{i}=\beta\left(e_{i}\right)$ for $1 \leq i \leq r$. Since $\pi$ is an isomorphism over $T$, each $v_{i} \neq 0$. As $\sigma$ is pointed, there exists some $p \in P$ such that $\left\langle v_{i}, p\right\rangle>0$ for all $i$. Viewing $p$ as an element of $F$ via the inclusion $P \subset F$, we have $\left\langle e_{i}, p\right\rangle>0$.

Let $f_{1}, \ldots, f_{r}$ be the basis of $\widetilde{M}$ dual to $e_{1}, \ldots, e_{r}$. Since the $f_{i}$ are generators of $F$, it suffices to prove the proposition for each $f_{i}$. Note that

$$
\left\langle e_{1}, p\right\rangle f_{1}+\cdots+\left\langle e_{r}, p\right\rangle f_{r}=p \in P .
$$

Since $\left\langle e_{i}, p\right\rangle>0$, we see that

$$
f_{i}^{\prime}:=\left(\left\langle e_{i}, p\right\rangle-1\right) f_{i}+\sum_{j \neq i}\left\langle e_{j}, p\right\rangle f_{j} \in F
$$

and that $f_{i}+f_{i}^{\prime} \in P$.

Proposition 2.15. Keep the notation and hypotheses of Proposition 2.14 and let $\beta: \widetilde{\sigma} \cap \widetilde{N} \rightarrow \sigma \cap N$ be the induced map. If $w \in \sigma \cap N$, then $\beta^{-1}(w)$ is a finite set.

Proof. Let $f_{1}, \ldots, f_{r}$ be the minimal generators of the monoid $F$. By Proposition 2.14, there exist $f_{1}^{\prime}, \ldots, f_{r}^{\prime}$ such that $f_{i}+f_{i}^{\prime} \in P$ for all $i \in\{1, \ldots, r\}$. For any $\widetilde{w} \in \beta^{-1}(w)$,

$$
\left\langle\widetilde{w}, f_{i}\right\rangle \leq\left\langle\widetilde{w}, f_{i}+f_{i}^{\prime}\right\rangle=\left\langle w, f_{i}+f_{i}^{\prime}\right\rangle
$$

so there are only finitely many possible values for each $\left\langle\widetilde{w}, f_{i}\right\rangle$. Thus $\beta^{-1}(w)$ is a finite set. 
We end this section by discussing canonical stacks as defined in [GS15a, Section 5].

Definition 2.16. If $\Sigma$ is a fan on a lattice $N$, let $v_{1}, \ldots, v_{r} \in N$ be the first lattice points on the rays of $\Sigma$, let $v: \mathbb{Z}^{r} \rightarrow N$ be the map $v\left(e_{i}\right):=v_{i}$ and let $\widetilde{\Sigma}$ be as in Definition 2.12. If $N^{\prime}$ is a direct complement of the support of $\Sigma$ and $v^{\prime}: \mathbb{Z}^{r} \oplus N^{\prime} \rightarrow N$ is given by $v^{\prime}\left(v, n^{\prime}\right)=v(v)+n^{\prime}$, then $\mathcal{X}_{\widetilde{\Sigma}, v^{\prime}}$ is the canonical stack of $X_{\Sigma}$.

Remark 2.17. With notation as in Definition 2.16, if the support of $\Sigma$ is $N$, the canonical stack of $X_{\Sigma}$ is the fantastack $\mathcal{F}_{\widetilde{\Sigma}, v}$.

The next proposition, which is straightforward from the definition, says that canonical stacks are compatible with open immersions. This will be useful for us, as this proposition will allow us to reduce most of our work to the case of affine toric varieties defined by a $d$-dimensional cone in $N_{\mathbb{R}}$.

Proposition 2.18. Let $\Sigma$ be a fan consisting of pointed rational cones in $N_{\mathbb{R}}$, let $\sigma$ be a cone in $\Sigma$, let $X(\Sigma)$ and $X(\sigma)$ be the T-toric varieties associated to $\Sigma$ and $\sigma$, respectively, and let $\iota: X(\sigma) \hookrightarrow X(\Sigma)$ be the open inclusion. If $\mathcal{X}(\Sigma)$ and $\mathcal{X}(\sigma)$ are the canonical stacks over $X(\Sigma)$ and $X(\sigma)$, respectively, and $\pi(\Sigma): \mathcal{X}(\Sigma) \rightarrow X(\Sigma)$ and $\pi(\sigma): \mathcal{X}(\sigma) \rightarrow X(\sigma)$ are the canonical maps, then there exists a map $\mathcal{X}(\sigma) \rightarrow \mathcal{X}(\Sigma)$ such that

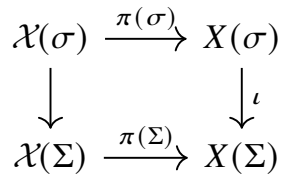

is a fibre product diagram.

For the remainder of this subsection, let $\sigma$ be a $d$-dimensional pointed rational cone in $N_{\mathbb{R}}$, let $X$ be the affine $T$-toric variety associated to $\sigma$, let $\mathcal{X}$ be the canonical stack over $X$ and let $\pi: \mathcal{X} \rightarrow X$ be the canonical map. At points later in this paper, we will refer to the following list of notations when we want to set it, and we also set it for the remainder of this subsection:

\section{Notation 2.19.}

○ Let $M=N^{*}$.

- Let $\widetilde{N}$ be the free abelian group with generators indexed by the rays of $\sigma$.

○ Let $\widetilde{M}=\widetilde{N}^{*}$.

○ Let $\langle\cdot, \cdot\rangle$ denote both pairings $N \otimes_{\mathbb{Z}} M \rightarrow \mathbb{Z}$ and $\widetilde{N} \otimes_{\mathbb{Z}} \widetilde{M} \rightarrow \mathbb{Z}$.

- Let $\widetilde{T}=\operatorname{Spec}(k[\widetilde{M}])$ be the algebraic torus with cocharacter lattice $\widetilde{N}$.

- Let $\widetilde{\sigma}$ be the positive orthant of $\widetilde{N}_{\mathbb{R}}-$ that is, $\widetilde{\sigma}$ is the positive span of those generators of $\widetilde{N}$ that are indexed by the rays of $\sigma$.

- Let $\widetilde{X}$ be the affine $\widetilde{T}$-toric variety associated to $\widetilde{\sigma}$.

- Let $\beta: \widetilde{\sigma} \cap \widetilde{N} \rightarrow \sigma \cap N$ be the monoid map taking the generator of $\widetilde{N}$ indexed by a ray of $\sigma$ to the first lattice point of that ray.

- Let $\widetilde{\pi}: \widetilde{X} \rightarrow X$ be the toric map associated to $\beta^{\mathrm{gp}}: \widetilde{N} \rightarrow N$.

○ Let $P=\sigma^{\vee} \cap M$. Note that $X=\operatorname{Spec}(k[P])$.

○ Let $F=\widetilde{\sigma}^{\vee} \cap \widetilde{M}$. Note that $\widetilde{X}=\operatorname{Spec}(k[F])$.

○ Identify $P$ with its image under the injection $P \hookrightarrow F$ given by dualising $\beta$. Note that $P \hookrightarrow F$ is injective because $\sigma$ is full-dimensional.

○ Let $A=F^{\mathrm{gp}} / P^{\mathrm{gp}}=\widetilde{M} / M$.

- Let $G=\operatorname{Spec}(k[A])$ be the kernel of the algebraic group homomorphism $\widetilde{T} \rightarrow T$ obtained by restricting $\widetilde{\pi}$, and let $G$ act on $\widetilde{X}$ by restricting the toric action of $\widetilde{T}$ on $\widetilde{X}$.

By definition, the canonical stack $\mathcal{X}$ is equal to the stack quotient $[\widetilde{X} / G]$ and the morphism $\tilde{\pi}: \widetilde{X} \rightarrow X$ is the composition $\widetilde{X} \rightarrow[\widetilde{X} / G]=\mathcal{X} \stackrel{\pi}{\rightarrow} X$. 
We note that because our focus is on singular varieties instead of on stacks, we simplify our exposition by focusing on canonical stacks over toric varieties instead of all fantastacks. The expositional advantage is that canonical stacks depend only on the toric variety and not on additional data, as is the case for other fantastacks. We end this section with two remarks which explain why we have not lost any generality by making this expositional simplification and discuss a generalisation of Theorem 1.7.

Remark 2.20. For Theorem 1.7, it is sufficient to consider canonical stacks, as these are precisely the fantastacks satisfying the hypotheses of Conjecture 1.1. Nonetheless, we note that with only superficial modifications to our techniques, one can actually prove a more general statement than Theorem 1.7, which we explain here.

With notation as in Definition 2.16, let $\mathcal{X}=\mathcal{F}_{\Sigma, v}$ be a fantastack. Assume that $X=X_{\Sigma}$ is $\mathbb{Q}$ Gorenstein, so for each maximal cone $\sigma \in \Sigma$, there exist $q_{\sigma} \in N^{*}$ and $m_{\sigma} \in \mathbb{Z}_{>0}$ such that the set

$$
\mathcal{H}_{\sigma}:=\left\{v \in \sigma \cap N \mid\left\langle q_{\sigma}, v\right\rangle=m_{\sigma}\right\}
$$

contains the first lattice point of every ray of $\sigma$. We say the good moduli space map $\pi: \mathcal{X} \rightarrow X$ is combinatorially crepant if $v\left(e_{i}\right) \in \bigcup_{\sigma} \mathcal{H}_{\sigma}$ for every $i \in\{1, \ldots, r\}$.

For example, the canonical stack is combinatorially crepant over $X$. Since Lemma 7.9 holds for all fantastacks that are combinatorially crepant over their good moduli space, the conclusions of Conjecture 1.1 hold for any fantastack that is combinatorially crepant over its good moduli space.

Remark 2.21. If $\mathcal{F}_{\Sigma, v}$ is a fantastack over $X$, then $\mathcal{F}_{\Sigma, v} \rightarrow X$ is an isomorphism over a nonempty open subset of $X$ if and only if $v$ does not send any standard basis vector to 0 . Since Proposition 2.14 holds for every fantastack satisfying the hypotheses of Conjecture 1.2, our proofs show that Theorem 1.11 holds for any fantastack as well.

\section{Motivic integration for stacks}

For the remainder of this paper, by a quotient stack over $k$ we will mean an Artin stack over $k$ that is isomorphic to the stack quotient of a $k$-scheme by the action of a linear algebraic group over $k$.

Remark 3.1. Let $G$ be a linear algebraic group over $k$ acting on a $k$-scheme $\widetilde{X}$, and let $G \hookrightarrow G^{\prime}$ be an inclusion of $G$ as a closed subgroup of a linear algebraic group $G^{\prime}$ over $k$. Then we have an isomorphism

$$
[\tilde{X} / G] \cong\left[\left(\widetilde{X} \times{ }^{G} G^{\prime}\right) / G^{\prime}\right]
$$

where $\widetilde{X} \times{ }^{G} G^{\prime}$ is the $k$-scheme with $G^{\prime}$-action obtained from $\widetilde{X}$ by pushout along $G \hookrightarrow G^{\prime}$. Thus any quotient stack is isomorphic to a stack quotient of a scheme by a general linear group, which in particular is a special group.

In this section we define a notion of motivic integration for quotient stacks. On the one hand, our definition is straightforward: it is more or less identical to motivic integration for schemes, but in various places we need to replace notions for schemes with the obvious analogues for Artin stacks; in particular, our motivic integration for quotient stacks does not depend on a choice of presentation for the stack as a quotient. On the other hand, our notion allows explicit computations in terms of motivic integration for schemes, as long as one first writes the stack as a stack quotient of a scheme by a special group.

Definition 3.2. Let $\mathcal{X}$ be an Artin stack over $k$, and set $n \in \mathbb{N}$. The $n$th jet stack of $\mathcal{X}$, denoted $\mathscr{L}_{n}(\mathcal{X})$, is the Weil restriction of $\mathcal{X} \otimes_{k} k[t] /\left(t^{n+1}\right)$ with respect to the morphism $\operatorname{Spec}\left(k[t] /\left(t^{n+1}\right)\right) \rightarrow \operatorname{Spec}(k)$.

Remark 3.3. Each jet stack $\mathscr{L}_{n}(\mathcal{X})$ is an Artin stack by [Ryd11, Theorem 3.7(iii)].

Remark 3.4. Each jet stack $\mathscr{L}_{n}(\mathcal{X})$ has affine (geometric) stabilisers by the following argument. Let $y: \operatorname{Spec}\left(k^{\prime}\right) \rightarrow \mathscr{L}_{n}(\mathcal{X})$ be a geometric point corresponding to $\psi_{n}: \operatorname{Spec}\left(k^{\prime}[t] /\left(t^{n+1}\right)\right) \rightarrow \mathcal{X}$. 
Because $\mathcal{X}$ has affine (geometric) stabilisers, the reduction of the stabiliser of $\psi_{n}$ is affine, so the stabiliser of $\psi_{n}$ is affine. Thus the stabiliser of $y$, which is the Weil restriction of the stabiliser of $\psi_{n}$, is affine.

The morphisms $k[t] /\left(t^{n+1}\right) \rightarrow k[t] /\left(t^{m+1}\right)$, when $n \geq m$, induce truncation morphisms $\theta_{m}^{n}:$ $\mathscr{L}_{n}(\mathcal{X}) \rightarrow \mathscr{L}_{m}(\mathcal{X})$ for any Artin stack $\mathcal{X}$ over $k$. Like in the case of schemes, we use these truncation morphisms to define arcs of $\mathcal{X}$ and a stack parametrising them.

Definition 3.5. Let $\mathcal{X}$ be an Artin stack over $k$. The arc stack of $\mathcal{X}$ is the inverse limit $\mathscr{L}(\mathcal{X})=$ $\lim _{n} \mathscr{L}_{n}(\mathcal{X})$, where the inverse limit is taken with respect to the truncation morphisms $\theta_{m}^{n}: \mathscr{L}_{n}(\mathcal{X}) \rightarrow$ $\overleftarrow{\mathscr{L}}_{m}^{n}(\mathcal{X})$

Remark 3.6. The name arc stack is justified by the fact that $\mathscr{L}(\mathcal{X})$ is indeed a stack (see, for example, [Tal14, Proposition 2.1.9]). Since $\mathscr{L}(\mathcal{X})$ is a stack as opposed to an Artin stack, we use the symbol $|\mathscr{L}(\mathcal{X})|$ to denote equivalence classes of points but do not define a topology on this set.

Remark 3.7. Let $\mathcal{X}$ be an Artin stack over $k$, and let $k^{\prime}$ be a field extension of $k$. The truncation morphism $k^{\prime} \llbracket t \rrbracket \rightarrow k^{\prime}[t] /\left(t^{n+1}\right)$ induces a functor $\mathcal{X}\left(k^{\prime} \llbracket t \rrbracket\right) \rightarrow \mathcal{X}\left(k^{\prime}[t] /\left(t^{n+1}\right)\right)=\mathscr{L}_{n}(\mathcal{X})\left(k^{\prime}\right)$ for each $n \in \mathbb{N}$, and these functors induce a functor $\mathcal{X}\left(k^{\prime} \llbracket t \rrbracket\right) \rightarrow \mathscr{L}(\mathcal{X})\left(k^{\prime}\right)$. Since $\mathcal{X}$ is an Artin stack, the functor $\mathcal{X}\left(k^{\prime} \llbracket t \rrbracket\right) \rightarrow \mathscr{L}(\mathcal{X})\left(k^{\prime}\right)$ is an equivalence of categories, for example by Artin's criterion for algebraicity. Throughout this paper, we will often implicitly make this identification.

We will let each $\theta_{n}: \mathscr{L}(\mathcal{X}) \rightarrow \mathscr{L}_{n}(\mathcal{X})$ denote the canonical morphism, and we will also call these truncation morphisms.

We will eventually define a notion of measurable subsets of $|\mathscr{L}(\mathcal{X})|$ and a motivic measure $\mu_{\mathcal{X}}$ that assigns an element of $\widehat{\mathscr{M}}_{k}$ to each of these measurable subsets. We begin with an important special case of measurable subsets. Note that when $\mathcal{X}$ is finite type over $k$, so is each $\mathscr{L}_{n}(\mathcal{X})$, by [Ryd11, Proposition $3.8(\mathrm{xv})]$.

Definition 3.8. Let $\mathcal{X}$ be a finite-type Artin stack over $k$, and set $\mathcal{C} \subset|\mathscr{L}(\mathcal{X})|$. We call the subset $\mathcal{C}$ a cylinder if there exist some $n \in \mathbb{N}$ and a constructible subset $\mathcal{C}_{n} \subset\left|\mathscr{L}_{n}(\mathcal{X})\right|$ such that $\mathcal{C}=\left(\theta_{n}\right)^{-1}\left(\mathcal{C}_{n}\right)$.

The next theorem, which we will prove later in this section, allows us to define a motivic integration for quotient stacks that is closely related to motivic integration for schemes.

Theorem 3.9. Let $\mathcal{X}$ be an equidimensional finite-type quotient stack over $k$, and let $\mathcal{C} \subset|\mathscr{L}(\mathcal{X})|$ be a cylinder. Then the set $\theta_{n}(\mathcal{C}) \subset\left|\mathscr{L}_{n}(\mathcal{X})\right|$ is constructible for each $n \in \mathbb{N}$, and the sequence

$$
\left\{\mathrm{e}\left(\theta_{n}(\mathcal{C})\right) \mathbb{L}^{-(n+1) \operatorname{dim} \mathcal{X}}\right\}_{n \in \mathbb{N}} \subset \widehat{\mathscr{M}}_{k}
$$

converges.

Furthermore, suppose that $G$ is a special group over $k$ and $\widetilde{X}$ is a $k$-scheme with $G$-action such that there exists an isomorphism $[\widetilde{X} / G] \stackrel{\sim}{\rightarrow} \mathcal{X}$, let $\rho: \widetilde{X} \rightarrow \mathcal{X}$ be the composition of the quotient map $\widetilde{X} \rightarrow[\widetilde{X} / G]$ with the isomorphism $[\widetilde{X} / G] \stackrel{\sim}{\rightarrow} \mathcal{X}$ and let $\widetilde{C}=\mathscr{L}(\rho)^{-1}(\mathcal{C})$. Then $\widetilde{C} \subset \mathscr{L}(\widetilde{X})$ is a cylinder, and

$$
\lim _{n \rightarrow \infty} \mathrm{e}\left(\theta_{n}(\mathcal{C})\right) \mathbb{L}^{-(n+1) \operatorname{dim} \mathcal{X}}=\mu_{\widetilde{X}}(\widetilde{C}) \mathrm{e}(G)^{-1} \mathbb{L}^{\operatorname{dim} G} \in \widehat{\mathscr{M}}_{k}
$$

Remark 3.10. Let $G$ and $\widetilde{X}$ be as in the statement of Theorem 3.9. Since $[\widetilde{X} / G]$ is equidimensional and $G$ is geometrically irreducible, $\widetilde{X}$ is equidimensional as well, and hence $\mu_{\widetilde{X}}$ is well defined. 
Before we prove Theorem 3.9, we will discuss some useful consequences. First, we can define the motivic measure $\mu_{\mathcal{X}}$ on cylinders.

Definition 3.11. Let $\mathcal{X}$ be an equidimensional finite-type quotient stack over $k$, and let $\mathcal{C} \subset|\mathscr{L}(\mathcal{X})|$ be a cylinder. The motivic measure of $\mathcal{C}$ is

$$
\mu_{\mathcal{X}}(\mathcal{C})=\lim _{n \rightarrow \infty} \mathrm{e}\left(\theta_{n}(\mathcal{C})\right) \mathbb{L}^{-(n+1) \operatorname{dim} \mathcal{X}} \in \widehat{\mathscr{M}}_{k}
$$

Remark 3.12. Let $\mathcal{X}$ be an equidimensional smooth Artin (not necessarily quotient) stack over $k$ and let $\mathcal{C} \subset|\mathscr{L}(\mathcal{X})|$ be a cylinder. One can verify that $\theta_{n}(\mathcal{C}) \subset\left|\mathscr{L}_{n}(\mathcal{X})\right|$ is constructible for each $n \in \mathbb{N}$ and that $\left\{\mathrm{e}\left(\theta_{n}(\mathcal{C})\right) \mathbb{L}^{-(n+1) \operatorname{dim} \mathcal{X}}\right\}_{n \in \mathbb{N}}$ stabilises for sufficiently large $n$, so Definition 3.11 also makes sense here. Although this is not used for the main results of this paper, our main conjectures are stated in the generality, so we provide the argument for completeness in subsection 3.5.

We now define measurable subsets analogously to the case of schemes.

Definition 3.13. Let $\mathcal{X}$ be an equidimensional finite-type quotient stack over $k$, set $\mathcal{C} \subset|\mathscr{L}(\mathcal{X})|$ and $\varepsilon \in \mathbb{R}_{>0}$, let $I$ be a set, let $\mathcal{C}^{(0)} \subset|\mathscr{L}(\mathcal{X})|$ be a cylinder and let $\left\{\mathcal{C}^{(i)}\right\}_{i \in I}$ be a collection of cylinders in $|\mathscr{L}(\mathcal{X})|$.

We say that $\left(\mathcal{C}^{(0)},\left(\mathcal{C}^{(i)}\right)_{i \in I}\right)$ is a cylindrical $\varepsilon$-approximation of $\mathcal{C}$ if

$$
\left(\mathcal{C} \cup \mathcal{C}^{(0)}\right) \backslash\left(\mathcal{C} \cap \mathcal{C}^{(0)}\right) \subset \bigcup_{i \in I} \mathcal{C}^{(i)}
$$

and, for all $i \in I$,

$$
\left\|\mu_{\mathcal{X}}\left(\mathcal{C}^{(i)}\right)\right\|<\varepsilon
$$

Definition 3.14. Let $\mathcal{X}$ be an equidimensional finite-type quotient stack over $k$, and set $\mathcal{C} \subset|\mathscr{L}(\mathcal{X})|$. We say that $\mathcal{C}$ is measurable if for any $\varepsilon \in \mathbb{R}_{>0}$, there exists a cylindrical $\varepsilon$-approximation of $\mathcal{C}$.

Remark 3.15. Let $\mathcal{X}$ be an equidimensional finite-type quotient stack over $k$, and let $\mathcal{C} \subset|\mathscr{L}(\mathcal{X})|$ be a cylinder. Then for any $\varepsilon \in \mathbb{R}_{>0}$, we have that $(\mathcal{C}, \emptyset)$ is a cylindrical $\varepsilon$-approximation of $\mathcal{C}$. In particular, $\mathcal{C}$ is measurable.

We now see that Theorem 3.9 allows us to extend $\mu_{\mathcal{X}}$ to measurable subsets.

Corollary 3.16. Let $\mathcal{X}$ be an equidimensional finite-type quotient stack over $k$, and let $\mathcal{C} \subset|\mathscr{L}(\mathcal{X})|$ be a measurable subset. Then there exists a unique $\mu_{\mathcal{X}}(\mathcal{C}) \in \widehat{\mathscr{M}}_{k}$ such that for any $\varepsilon \in \mathbb{R}_{>0}$ and any cylindrical $\varepsilon$-approximation $\left(\mathcal{C}^{(0)},\left(\mathcal{C}^{(i)}\right)_{i \in I}\right)$ of $\mathcal{C}$,

$$
\left\|\mu_{\mathcal{X}}(\mathcal{C})-\mu_{\mathcal{X}}\left(\mathcal{C}^{(0)}\right)\right\|<\varepsilon
$$

Furthermore, suppose that $G, \widetilde{X}, \rho$ are as in the statement of Theorem 3.9 and $\widetilde{C}=\mathscr{L}(\rho)^{-1}(\mathcal{C})$. Then $\widetilde{C} \subset \mathscr{L}(\widetilde{X})$ is measurable, and

$$
\mu_{\mathcal{X}}(\mathcal{C})=\mu_{\widetilde{X}}(\widetilde{C}) \mathrm{e}(G)^{-1} \mathbb{L}^{\operatorname{dim} G} \in \widehat{M}_{k}
$$

Proof. Let $G, \widetilde{X}, \rho, \widetilde{C}$ be as in the second part of the corollary. For any $\varepsilon \in \mathbb{R}_{>0}$ and any cylindrical $\varepsilon$-approximation $\left(\mathcal{C}^{(0)},\left(\mathcal{C}^{(i)}\right)_{i \in I}\right)$ of $\mathcal{C}$, Theorem 3.9 implies that $\left(\mathscr{L}(\rho)^{-1}\left(\mathcal{C}^{(0)}\right),\left(\mathscr{L}(\rho)^{-1}\left(\mathcal{C}^{(i)}\right)\right)_{i \in I}\right)$ 
is a cylindrical $\varepsilon\left\|\mathrm{e}(G) \mathbb{L}^{-\operatorname{dim} G}\right\|$-approximation of $\widetilde{C}$. Thus $\widetilde{C}$ is measurable, and for any cylindrical $\varepsilon$-approximation $\left(\mathcal{C}^{(0)},\left(\mathcal{C}^{(i)}\right)_{i \in I}\right)$ of $\mathcal{C}$

$$
\begin{aligned}
\| \mu_{\widetilde{X}}(\widetilde{C}) & \mathrm{e}(G)^{-1} \mathbb{L}^{\operatorname{dim} G}-\mu_{\mathcal{X}}\left(\mathcal{C}^{(0)}\right) \| \\
& =\left\|\mu_{\widetilde{X}}(\widetilde{C}) \mathrm{e}(G)^{-1} \mathbb{L}^{\operatorname{dim} G}-\mu_{\widetilde{X}}\left(\mathscr{L}(\rho)^{-1}\left(\mathcal{C}^{(0)}\right)\right) \mathrm{e}(G)^{-1} \mathbb{L}^{\operatorname{dim} G}\right\| \\
& \leq\left\|\mathrm{e}(G)^{-1} \mathbb{L}^{\operatorname{dim} G}\right\|\left\|\mu_{\widetilde{X}}(\widetilde{C})-\mu_{\widetilde{X}}\left(\mathscr{L}(\rho)^{-1}\left(\mathcal{C}^{(0)}\right)\right)\right\| \\
& <\varepsilon\left\|\mathrm{e}(G)^{-1} \mathbb{L}^{\operatorname{dim} G}\right\|\left\|\mathrm{e}(G) \mathbb{L}^{-\operatorname{dim} G}\right\|,
\end{aligned}
$$

where the first equality follows from Theorem 3.9. Once $\mu_{\mathcal{X}}(\mathcal{C})$ is shown to exist, this chain of inequalities proves $\mu_{\mathcal{X}}(\mathcal{C})=\mu_{\widetilde{X}}(\widetilde{C}) \mathrm{e}(G)^{-1} \mathbb{L}^{\operatorname{dim} G}$. To show the existence of $\mu_{\mathcal{X}}(\mathcal{C})$, it suffices by Remark 3.1 to assume $G$ is a general linear group, so this chain of inequalities and Lemma 3.17 finish the proof.

Lemma 3.17. Let $G$ be a general linear group over $k$. Then

$$
\left\|\mathrm{e}(G)^{-1} \mathbb{L}^{\operatorname{dim} G}\right\|\left\|\mathrm{e}(G) \mathbb{L}^{-\operatorname{dim} G}\right\|=1 .
$$

Proof. Using Euler-Poincaré polynomials, it is straightforward to check (see, for example, the proof of [CLNS18, Chapter 2, Lemma 4.1.3]) that if $n_{0} \in \mathbb{Z}$ and $\left\{c_{n}\right\}_{n \geq n_{0}}$ is a sequence of integers with $c_{n_{0}} \neq 0$, then

$$
\left\|\sum_{n \geq n_{0}} c_{n} \mathbb{L}^{-n}\right\|=\exp \left(-n_{0}\right) .
$$

The lemma then follows from the fact that $\mathrm{e}(G)$ is a polynomial in $\mathbb{L}$ (see, for example, the proof of [Joy07, Lemma 4.6]).

Definition 3.18. Let $\mathcal{X}$ be an equidimensional finite-type quotient stack over $k$, and let $\mathcal{C} \subset|\mathscr{L}(\mathcal{X})|$ be a measurable subset. The motivic measure of $\mathcal{C}$ is defined to be $\mu_{\mathcal{X}}(\mathcal{C}) \in \widehat{\mathscr{M}}_{k}$, as in the statement of Corollary 3.16 .

Remark 3.19. Remark 3.15 implies that Definition 3.18 generalises Definition 3.11 .

In the next two subsections, we will prove Theorem 3.9.

\subsection{Jet schemes of quotient stacks}

In this subsection, we describe the jet schemes of a stack quotient as stack quotients themselves. This is the first step in providing the relationship between motivic integration for quotient stacks and motivic integration for schemes. This description, Corollary 3.22, is a special case of the next proposition, which describes the Weil restriction of a stack quotient.

If $S^{\prime}$ and $S$ are schemes and $S^{\prime} \rightarrow S$ is a finite flat morphism of finite presentation, we will let $\mathscr{R}_{S^{\prime} / S}$ denote the functor taking each stack over $S^{\prime}$ to its Weil restriction with respect to $S^{\prime} \rightarrow S$, and we note that if $\mathcal{X}$ is an Artin stack over $S^{\prime}$, then $\mathscr{R}_{S^{\prime} / S}(\mathcal{X})$ is an Artin stack over $S$ [Ryd11, Theorem 3.7(iii)].

Proposition 3.20. Let $S^{\prime}$ and $S$ be schemes and $S^{\prime} \rightarrow S$ be a finite flat morphism of finite presentation. If $\widetilde{X}^{\prime}$ is an $S^{\prime}$-scheme with an action by a linear algebraic group $G^{\prime}$ over $S^{\prime}$, then there exists an isomorphism

$$
\mathscr{R}_{S^{\prime} / S}\left(\left[\widetilde{X}^{\prime} / G^{\prime}\right]\right) \stackrel{\sim}{\rightarrow}\left[\mathscr{R}_{S^{\prime} / S}\left(\widetilde{X}^{\prime}\right) / \mathscr{R}_{S^{\prime} / S}\left(G^{\prime}\right)\right]
$$


such that

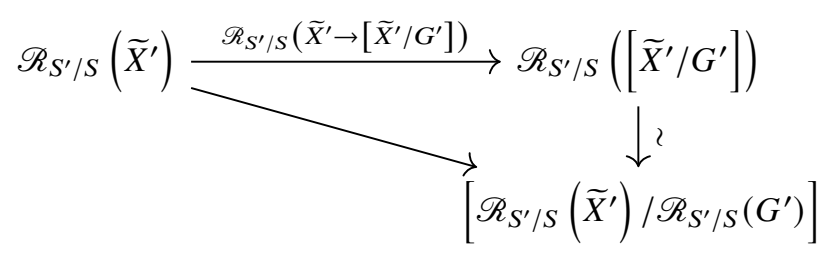

commutes.

Remark 3.21. In the statement of Proposition 3.20, the action of $\mathscr{R}_{S^{\prime} / S}\left(G^{\prime}\right)$ on $\mathscr{R}_{S^{\prime} / S}\left(\widetilde{X}^{\prime}\right)$ is obtained by applying $\mathscr{R}_{S^{\prime} / S}$ to the map $G^{\prime} \times_{S^{\prime}} \widetilde{X}^{\prime} \rightarrow \widetilde{X}^{\prime}$ defining the action of $G^{\prime}$ on $\widetilde{X}^{\prime}$.

Proof. We let $\mathcal{X}^{\prime}=\left[\widetilde{X}^{\prime} / G^{\prime}\right], \rho^{\prime}: \widetilde{X}^{\prime} \rightarrow \mathcal{X}^{\prime}$ be the quotient map, $\mathcal{X}=\mathscr{R}_{S^{\prime} / S}\left(\mathcal{X}^{\prime}\right), \widetilde{X}=\mathscr{R}_{S^{\prime} / S}\left(\widetilde{X}^{\prime}\right)$ and $G=\mathscr{R}_{S^{\prime} / S}\left(G^{\prime}\right)$. Since $\rho^{\prime}: \widetilde{X}^{\prime} \rightarrow \mathcal{X}^{\prime}$ is a smooth cover, $\mathscr{R}_{S^{\prime} / S}\left(\rho^{\prime}\right): \widetilde{X} \rightarrow \mathcal{X}$ is as well, by [Ryd11, Proposition 3.5(v)]. Since $\rho^{\prime}$ is a $G^{\prime}$-torsor, the natural map $G^{\prime} \times{ }_{S^{\prime}} \widetilde{X}^{\prime} \rightarrow \widetilde{X}^{\prime} \times \mathcal{X}^{\prime} \widetilde{X}^{\prime}$ induced by the $G^{\prime}$-action $G^{\prime} \times_{S^{\prime}} \widetilde{X}^{\prime} \rightarrow \widetilde{X}^{\prime}$ is an isomorphism, and applying Weil restriction, we see that the map $G \times_{S} \widetilde{X} \rightarrow \widetilde{X} \times_{\mathcal{X}} \widetilde{X}$ induced by the $G$-action $G \times_{S} \widetilde{X} \rightarrow \widetilde{X}$ is an isomorphism as well. Thus, $\mathscr{R}_{S^{\prime} / S}\left(\rho^{\prime}\right): \widetilde{X} \rightarrow \mathcal{X}$ is a $G$-torsor, thereby inducing an isomorphism $\mathcal{X} \stackrel{\sim}{\rightarrow}[\widetilde{X} / G]$ which makes the diagram in the statement of the proposition commute.

By the definition of jet stacks, the following is a special case of Proposition 3.20:

Corollary 3.22. Let $G$ be a linear algebraic group over $k$ acting on a $k$-scheme $\widetilde{X}$, and set $n \in \mathbb{N}$. There exists an isomorphism

$$
\mathscr{L}_{n}([\widetilde{X} / G]) \stackrel{\sim}{\rightarrow}\left[\mathscr{L}_{n}(\widetilde{X}) / \mathscr{L}_{n}(G)\right]
$$

such that

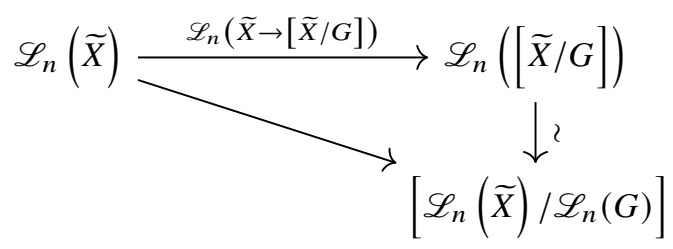

commutes.

Remark 3.23. In the statement of Corollary 3.22, the action of $\mathscr{L}_{n}(G)$ on $\mathscr{L}_{n}(\widetilde{X})$ is obtained by applying $\mathscr{L}_{n}$ to the map $G \times_{k} \widetilde{X} \rightarrow \widetilde{X}$ defining the $G$-action on $\widetilde{X}$.

\subsection{Truncation morphisms and quotient stacks}

Lemma 3.24. Let $\mathcal{X}$ be an Artin stack over $k$, let $\widetilde{X}$ be a scheme over $k$ and let $\rho: \widetilde{X} \rightarrow \mathcal{X}$ be a smooth covering. Set $\mathcal{C} \subset|\mathscr{L}(\mathcal{X})|$ and $\widetilde{C}=\mathscr{L}(\rho)^{-1}(\mathcal{C}) \subset \mathscr{L}(\widetilde{X})$. Then for all $n \in \mathbb{N}$,

$$
\mathscr{L}_{n}(\rho)^{-1}\left(\theta_{n}(\mathcal{C})\right)=\theta_{n}(\widetilde{C})
$$

Proof. Set $n \in \mathbb{N}$. Clearly, $\theta_{n}(\widetilde{C}) \subset \mathscr{L}_{n}(\rho)^{-1}\left(\theta_{n}(\mathcal{C})\right)$. 
To prove the opposite inclusion, let $k^{\prime}$ be a field extension of $k$, and let $\widetilde{\psi}_{n} \in \mathscr{L}_{n}(\widetilde{X})\left(k^{\prime}\right)$ and $\psi \in \mathscr{L}(\mathcal{X})\left(k^{\prime}\right)$ be such that the class of $\psi$ in $|\mathscr{L}(\mathcal{X})|$ is contained in $\mathcal{C}$ and $\mathscr{L}_{n}(\rho)\left(\widetilde{\psi}_{n}\right) \cong \theta_{n}(\psi)$. We must show $\widetilde{\psi}_{n} \in \theta_{n}(\widetilde{C})$. Since $\rho$ is smooth, by the infinitesimal lifting criterion, we have a dotted arrow filling in the following diagram:

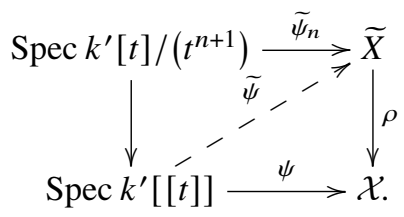

Then $\widetilde{\psi} \in \widetilde{C}$, so $\widetilde{\psi}_{n} \in \theta_{n}(\widetilde{C})$.

We may now prove the next proposition, which by Remark 3.1 and Remark 3.10 implies Theorem 3.9. Proposition 3.25. Let $G$ be a special group over $k$, let $\widetilde{X}$ be an equidimensional finite-type scheme over $k$ with $G$-action, let $\mathcal{X}=[\widetilde{X} / G]$, let $\rho: \widetilde{X} \rightarrow \mathcal{X}$ be the quotient map, let $\mathcal{C} \subset|\mathscr{L}(\mathcal{X})|$ be a cylinder and let $\widetilde{C}=\mathscr{L}(\rho)^{-1}(\mathcal{C})$. Then $\widetilde{C} \subset \mathscr{L}(\widetilde{X})$ is a cylinder, the set $\theta_{n}(\mathcal{C}) \subset\left|\mathscr{L}_{n}(\mathcal{X})\right|$ is constructible for each $n \in \mathbb{N}$, and the sequence

$$
\left\{\mathrm{e}\left(\theta_{n}(\mathcal{C})\right) \mathbb{L}^{-(n+1) \operatorname{dim} \mathcal{X}}\right\}_{n \in \mathbb{N}} \subset \widehat{\mathscr{M}}_{k}
$$

converges to

$$
\mu_{\widetilde{X}}(\widetilde{C}) \mathrm{e}(G)^{-1} \mathbb{L}^{\operatorname{dim} G} \in \widehat{\mathscr{M}}_{k}
$$

Remark 3.26. In the statement of Proposition 3.25, because $G$ is irreducible, the irreducible components of $\widetilde{X}$ are $G$-invariant, so $\mathcal{X}$ is equidimensional.

Proof. We first show that $\widetilde{C} \subset \mathscr{L}(\widetilde{X})$ is a cylinder. Because $\mathcal{C}$ is a cylinder, there exist some $n \in \mathbb{N}$ and some constructible subset $\mathcal{C}_{n} \subset\left|\mathscr{L}_{n}(\mathcal{X})\right|$ such that $\mathcal{C}=\left(\theta_{n}\right)^{-1}\left(\mathcal{C}_{n}\right)$. Then $\widetilde{C}=\left(\theta_{n}\right)^{-1}\left(\mathscr{L}_{n}(\rho)^{-1}\left(\mathcal{C}_{n}\right)\right)$ is a cylinder.

Now we will show that for all $n \in \mathbb{N}$, the set $\theta_{n}(\mathcal{C})$ is a constructible subset of $\mathscr{L}_{n}(\mathcal{X})$. Each $\theta_{n}(\widetilde{C})$ is a constructible subset of $\mathscr{L}_{n}(\widetilde{X})$. Therefore each $\theta_{n}(\mathcal{C}) \subset\left|\mathscr{L}_{n}(\mathcal{X})\right|$ is constructible, by Chevalley's theorem for Artin stacks [HR17, Theorem 5.2], Corollary 3.22 and Lemma 3.24.

Then since $G$ is a special group, $\mathscr{L}_{n}(G)$ is as well, by Remark 2.4. Then Corollary 3.22 and Lemma 3.24 imply that for each $n \in \mathbb{N}$,

$$
\mathrm{e}\left(\theta_{n}(\mathcal{C})\right)=\mathrm{e}\left(\theta_{n}(\widetilde{C})\right) \mathrm{e}\left(\mathscr{L}_{n}(G)\right)^{-1}=\mathrm{e}\left(\theta_{n}(\widetilde{C})\right) \mathrm{e}(G)^{-1} \mathbb{L}^{-n \operatorname{dim} G}
$$

where the second equality holds because $G$ is smooth. Therefore,

$$
\begin{aligned}
\mu_{\widetilde{X}}(\widetilde{C}) \mathrm{e}(G)^{-1} \mathbb{L}^{\operatorname{dim} G} & =\lim _{n \rightarrow \infty} \mathrm{e}\left(\theta_{n}(\widetilde{C})\right) \mathrm{e}(G)^{-1} \mathbb{L}^{\operatorname{dim} G-(n+1) \operatorname{dim} \widetilde{X}} \\
& =\lim _{n \rightarrow \infty} \mathrm{e}\left(\theta_{n}(\mathcal{C})\right) \mathbb{L}^{-(n+1) \operatorname{dim} \mathcal{X}}
\end{aligned}
$$




\subsection{Properties of motivic integration for quotient stacks}

We now state some basic properties of motivic integration for quotient stacks. We will use these properties later in this paper.

Proposition 3.27. Let $\mathcal{X}$ be an equidimensional finite-type quotient stack over $k$, let $\left\{\mathcal{C}^{(i)}\right\}_{i \in \mathbb{N}}$ be a sequence of pairwise disjoint measurable subsets of $|\mathscr{L}(\mathcal{X})|$ and let $\mathcal{C}=\bigcup_{i=0}^{\infty} \mathcal{C}^{(i)} \cdot \operatorname{Iflim}_{i \rightarrow \infty} \mu_{\mathcal{X}}\left(\mathcal{C}^{(i)}\right)=0$, then $\mathcal{C}$ is measurable and

$$
\mu_{\mathcal{X}}(\mathcal{C})=\sum_{i=0}^{\infty} \mu_{\mathcal{X}}\left(\mathcal{C}^{(i)}\right)
$$

Proof. The set $\mathcal{C}$ is measurable by the exact same proof used for the analogous statement for schemes in [CLNS18, Chapter 6, Proposition 3.4.2]. The remainder of the proposition follows from Corollary 3.16 and the analogous statement for schemes [CLNS18, Chapter 6, Proposition 3.4.3] applied to the scheme $\widetilde{X}$ in the statement of Corollary 3.16.

Proposition 3.28. Let $\mathcal{X}$ be an equidimensional finite-type quotient stack over $k$, and set $\mathcal{C} \subset \mathcal{D} \subset$ $|\mathscr{L}(\mathcal{X})|$. If $\mathcal{D}$ is measurable and $\mu_{\mathcal{X}}(\mathcal{D})=0$, then $\mathcal{C}$ is measurable and $\mu_{\mathcal{X}}(\mathcal{C})=0$.

Proof. The proposition holds by the exact same proof used for the analogous statement for schemes in [CLNS18, Chapter 6, Corollary 3.5.5(a)].

Proposition 3.29. Let $\mathcal{X}$ be an equidimensional finite-type quotient stack over $k$, and let $\mathcal{C}, \mathcal{D}$ be measurable subsets of $|\mathscr{L}(\mathcal{X})|$. If $\mathcal{C} \subset \mathcal{D}$, then

$$
\left\|\mu_{\mathcal{X}}(\mathcal{C})\right\| \leq\left\|\mu_{\mathcal{X}}(\mathcal{D})\right\|
$$

Proof. By Remark 3.1, there exist $G, \widetilde{X}, \rho$ as in the statement of Theorem 3.9 such that $G$ is a general linear group. Let $\widetilde{C}=\mathscr{L}(\rho)^{-1}(\mathcal{C})$ and $\widetilde{D}=\mathscr{L}(\rho)^{-1}(\mathcal{D})$. Then

$$
\begin{aligned}
\left\|\mu_{\mathcal{X}}(\mathcal{C})\right\| & =\left\|\mu_{\widetilde{X}}(\widetilde{C}) \mathrm{e}(G)^{-1} \mathbb{L}^{\operatorname{dim} G}\right\| \\
& \leq\left\|\mathrm{e}(G)^{-1} \mathbb{L}^{\operatorname{dim} G}\right\|\left\|\mu_{\tilde{X}}(\widetilde{C})\right\| \\
& \leq\left\|\mathrm{e}(G)^{-1} \mathbb{L}^{\operatorname{dim} G}\right\|\left\|\mu_{\widetilde{X}}(\widetilde{D})\right\| \\
& =\left\|\mathrm{e}(G)^{-1} \mathbb{L}^{\operatorname{dim} G}\right\|\left\|\mu_{\mathcal{X}}(\mathcal{D}) \mathrm{e}(G) \mathbb{L}^{-\operatorname{dim} G}\right\| \\
& \leq\left\|\mathrm{e}(G)^{-1} \mathbb{L}^{\operatorname{dim} G}\right\|\left\|\mathrm{e}(G) \mathbb{L}^{-\operatorname{dim} G}\right\|\left\|\mu_{\mathcal{X}}(\mathcal{D})\right\| \\
& =\left\|\mu_{\mathcal{X}}(\mathcal{D})\right\|,
\end{aligned}
$$

where the first and fourth lines follow from Corollary 3.16, the third line follows from the analogous statement [CLNS18, Chapter 6, Corollary 3.3.5] for schemes applied to $\widetilde{X}$ and the last line follows from Lemma 3.17.

Proposition 3.30. Let $\mathcal{X}$ be an equidimensional finite-type quotient stack over $k$, let $\mathcal{Y}$ be a closed substack of $\mathcal{X}$ with $\operatorname{dim} \mathcal{Y}<\operatorname{dim} \mathcal{X}$ and let $\mathcal{C} \subset|\mathscr{L}(\mathcal{X})|$ be the image of $|\mathscr{L}(\mathcal{Y})|$ in $|\mathscr{L}(\mathcal{X})|$. Then $\mathcal{C}$ is measurable and $\mu_{\mathcal{X}}(\mathcal{C})=0$.

Proof. For each $n \in \mathbb{N}$, let $\mathcal{C}_{n} \subset\left|\mathscr{L}_{n}(\mathcal{X})\right|$ be the image of $\left|\mathscr{L}_{n}(\mathcal{Y})\right|$ in $\left|\mathscr{L}_{n}(\mathcal{X})\right|$, and let $\mathcal{C}^{(n)}=\left(\theta_{n}\right)^{-1}\left(\mathcal{C}_{n}\right)$. By [Ryd11, Proposition 3.5(vi)], each $\mathcal{C}_{n}$ is a closed subset of $\mathscr{L}_{n}(\mathcal{X})$, so each $\mathcal{C}^{(n)}$ is a cylinder in $\mathscr{L}(\mathcal{X})$. 
By Remark 3.1, there exist $G, \widetilde{X}, \rho$ as in the statement of Theorem 3.9. Let $\widetilde{Y}=\widetilde{X} \times \mathcal{X} \mathcal{Y}$. Then $\mathscr{L}_{n}(\rho)^{-1}\left(\mathcal{C}_{n}\right)$ is the underlying set of $\mathscr{L}_{n}(\widetilde{Y})$. Thus by Theorem 3.9,

$$
\begin{aligned}
\mu_{\mathcal{X}}\left(\mathcal{C}^{(n)}\right) & =\mu_{\widetilde{X}}\left(\mathscr{L}(\rho)^{-1}\left(\mathcal{C}^{(n)}\right)\right) \mathrm{e}(G)^{-1} \mathbb{L}^{\operatorname{dim} G} \\
& =\mu_{\widetilde{X}}\left(\left(\theta_{n}\right)^{-1}\left(\mathscr{L}_{n}(\widetilde{Y})\right)\right) \mathrm{e}(G)^{-1} \mathbb{L}^{\operatorname{dim} G}
\end{aligned}
$$

By [CLNS18, Chapter 6, Proposition 2.3.1],

$$
\lim _{n \rightarrow \infty} \mu_{\widetilde{X}}\left(\left(\theta_{n}\right)^{-1}\left(\mathscr{L}_{n}(\widetilde{Y})\right)\right)=0
$$

so

$$
\lim _{n \rightarrow \infty} \mu_{\mathcal{X}}\left(\mathcal{C}^{(n)}\right)=0
$$

Therefore for any $\varepsilon \in \mathbb{R}_{>0}$, we get that $\left(\emptyset,\left(\mathcal{C}^{(n)}\right)\right)$ is a cylindrical $\varepsilon$-approximation of $\mathcal{C}$ for sufficiently large $n$, and we are done, by the definition of $\mu_{\mathcal{X}}$.

Proposition 3.31. Let $\mathcal{X}$ be an equidimensional finite-type quotient stack over $k$, and let $\mathcal{C}$ and $\mathcal{D}$ be measurable subsets of $|\mathscr{L}(\mathcal{X})|$. Then the intersection $\mathcal{C} \cap \mathcal{D}$, the union $\mathcal{C} \cup \mathcal{D}$ and the complement $\mathcal{C} \backslash \mathcal{D}$ are all measurable subsets of $|\mathscr{L}(\mathcal{X})|$.

Proof. The proposition holds by the exact same proof used for the analogous statement for schemes in [CLNS18, Chapter 6, Proposition 3.2.8].

Proposition 3.32. Let $\mathcal{X}$ be an equidimensional finite-type quotient stack over $k$, let $\iota: \mathcal{U} \hookrightarrow \mathcal{X}$ be the inclusion of an open substack and set $\mathcal{C} \subset\left(\theta_{0}\right)^{-1}(|\mathcal{U}|) \subset|\mathscr{L}(\mathcal{X})|$. Then $\mathcal{C}$ is a measurable subset of $|\mathscr{L}(\mathcal{X})|$ if and only if $\mathscr{L}(\iota)^{-1}(\mathcal{C})$ is a measurable subset of $|\mathscr{L}(\mathcal{U})|$, and in that case,

$$
\mu_{\mathcal{X}}(\mathcal{C})=\mu_{\mathcal{U}}\left(\mathscr{L}(\iota)^{-1}(\mathcal{C})\right)
$$

Proof. As in the case of schemes, this is an easy consequence of the definitions and the fact that for all $n \in \mathbb{N}$, the morphism $\mathscr{L}_{n}(\iota): \mathscr{L}_{n}(\mathcal{U}) \rightarrow \mathscr{L}_{n}(\mathcal{X})$ is an open immersion by [Ryd11, Proposition 3.5(vii)].

\subsection{Nonseparatedness functions}

We now introduce notation for the nonseparatedness functions $\operatorname{sep}_{\pi, \mathcal{C}}, \operatorname{sep}_{\pi}$ and $\operatorname{sep}_{\mathcal{X}}$ that were used in the statements of the main conjectures and theorems of this paper. Throughout this subsection, let $\mathcal{X}$ be an Artin stack over $k$, set $\mathcal{C} \subset|\mathscr{L}(\mathcal{X})|$, let $X$ be a scheme over $k$ and let $\pi: \mathcal{X} \rightarrow X$ be a map. For any field extension $k^{\prime}$ of $k$, we will let $\overline{\mathcal{C}}\left(k^{\prime}\right)$ denote the subset of $\overline{\mathscr{L}(\mathcal{X})}\left(k^{\prime}\right)$ consisting of arcs whose classes in the set $|\mathscr{L}(\mathcal{X})|$ are contained in $\mathcal{C}$.

If $k^{\prime}$ is a field extension of $k$ and $\varphi \in \mathscr{L}(X)\left(k^{\prime}\right)$, we set

$$
\operatorname{sep}_{\pi, \mathcal{C}}(\varphi)=\#\left(\overline{\mathcal{C}}\left(k^{\prime}\right) \cap \overline{\left(\mathscr{L}(\pi)^{-1}(\varphi)\right)}\left(k^{\prime}\right)\right) \in \mathbb{N} \cup\{\infty\}
$$

which induces a map $\operatorname{sep}_{\pi, \mathcal{C}}: \mathscr{L}(X) \rightarrow \mathbb{N} \cup\{\infty\}$ by considering each $\varphi \in \mathscr{L}(X)$ as a point valued in its residue field. If, furthermore, we assume that $X$ is integral, finite type and separated and has 
log-terminal singularities, that $\operatorname{sep}_{\pi, \mathcal{C}}: \mathscr{L}(X) \rightarrow \mathbb{N} \cup\{\infty\}$ has measurable fibres and that $C \subset \mathscr{L}(X)$ is a measurable subset, then we can consider the motivic integral

$$
\int_{C} \operatorname{sep}_{\pi, \mathcal{C}} \mathrm{d} \mu_{X}^{\mathrm{Gor}}=\sum_{n \in \mathbb{N}} n \mu_{X}^{\mathrm{Gor}}\left(\operatorname{sep}_{\pi, \mathcal{C}}^{-1}(n) \cap C\right) \in \widehat{\mathscr{M}}_{k}\left[\mathbb{L}^{1 / m}\right],
$$

where $m \in \mathbb{Z}_{>0}$ is such that $m K_{X}$ is Cartier. Note that with these assumptions, the series defining $\int_{C} \operatorname{sep}_{\pi, \mathcal{C}} \mathrm{d} \mu_{X}^{\text {Gor }}$ converges because

$$
\lim _{n \rightarrow \infty} n \mu_{X}^{\mathrm{Gor}}\left(\operatorname{sep}_{\pi, \mathcal{C}}^{-1}(n) \cap C\right)=0
$$

which follows from

$$
\lim _{n \rightarrow \infty} \mu_{X}^{\mathrm{Gor}}\left(\operatorname{sep}_{\pi, \mathcal{C}}^{-1}(n) \cap C\right)=0
$$

which itself, for example, is a consequence of Proposition 2.3.

Set

$$
\operatorname{sep}_{\pi}=\operatorname{sep}_{\pi,|\mathscr{L}(\mathcal{X})|}
$$

and

$$
\operatorname{sep}_{\mathcal{X}}=1 /\left(\operatorname{sep}_{\pi} \circ \mathscr{L}(\pi)\right):|\mathscr{L}(\mathcal{X})| \rightarrow \mathbb{Q}_{\geq 0} \cup\{\infty\}
$$

If, furthermore, we assume that $\mathcal{X}$ is an equidimensional and finite-type quotient stack over $k$ and that $\operatorname{sep}_{\mathcal{X}}:|\mathscr{L}(\mathcal{X})| \rightarrow \mathbb{Q}_{\geq 0} \cup\{\infty\}$ has measurable fibres, we can consider the motivic integral

$$
\int_{\mathscr{L}(\mathcal{X})} \operatorname{sep}_{\mathcal{X}} \mathrm{d} \mu_{\mathcal{X}}=\sum_{n \in \mathbb{Z}_{\geq 1}}(1 / n) \mu_{\mathcal{X}}\left(\operatorname{sep}_{\mathcal{X}}^{-1}(1 / n)\right) \in \overline{\mathscr{M}_{k} \otimes_{\mathbb{Z}} \mathbb{Q}}
$$

where the ring $\overline{\mathscr{M}_{k} \otimes_{\mathbb{Z}} \mathbb{Q}}$ is defined like $\widehat{\mathscr{M}}_{k}$ in subsection 2.1 by replacing any mention of $K_{0}\left(\operatorname{Var}_{k}\right)$ with $K_{0}\left(\operatorname{Var}_{k}\right) \otimes_{\mathbb{Z}} \mathbb{Q}$ and any mention of 'subgroup' with ' $\mathbb{Q}$-subspace'. With these assumptions, the series defining $\int_{\mathscr{L}(\mathcal{X})} \operatorname{sep}_{\mathcal{X}} \mathrm{d} \mu_{\mathcal{X}}$ converges because

$$
\lim _{n \rightarrow \infty}(1 / n) \mu_{\mathcal{X}}\left(\operatorname{sep}_{\mathcal{X}}^{-1}(1 / n)\right)=0
$$

which by the definition of the norm on $\overline{\bar{M}_{k} \otimes_{\mathbb{Z}} \mathbb{Q}}$ follows from

$$
\lim _{n \rightarrow \infty} \mu_{\mathcal{X}}\left(\operatorname{sep}_{\mathcal{X}}^{-1}(1 / n)\right)=0
$$

which itself follows from Corollary 3.16 and properties of motivic measures for schemes.

\subsection{Motivic integration for smooth stacks via the cotangent complex}

In this subsection, we prove that the motivic measure $\mu_{\mathcal{X}}$ is also well defined when $\mathcal{X}$ is an equidimensional smooth Artin (not necessarily quotient) stack over $k$. We only explicitly verify this for cylinders, but by a standard argument (identical to the one for schemes in [CLNS18]), it leads to well-defined notions (that coincide with our definitions in the case where $\mathcal{X}$ is a quotient stack) of measurable subsets of $|\mathscr{L}(\mathcal{X})|$ and their motivic measures. The main result of this subsection is the following theorem, which immediately implies that Definition 3.11 makes sense in this setting: 
Theorem 3.33. Let $\mathcal{X}$ be an equidimensional smooth Artin stack over $k$, and let $\mathcal{C} \subset|\mathscr{L}(\mathcal{X})|$ be a cylinder. Then the set $\theta_{n}(\mathcal{C}) \subset\left|\mathscr{L}_{n}(\mathcal{X})\right|$ is constructible for each $n \in \mathbb{N}$, and the sequence

$$
\left\{\mathrm{e}\left(\theta_{n}(\mathcal{C})\right) \mathbb{L}^{-(n+1) \operatorname{dim} \mathcal{X}}\right\}_{n \in \mathbb{N}} \subset K_{0}\left(\mathbf{S t a c k}_{k}\right)
$$

stabilises for sufficiently large $n$.

We first prove two lemmas, after which we prove this theorem.

Lemma 3.34. Let $\mathcal{X}$ be an equidimensional smooth Artin stack over $k$, and set $n \in \mathbb{N}$. There exist some $\ell \in \mathbb{Z}_{>0}$, a partition $\left|\mathscr{L}_{n}(\mathcal{X})\right|=\bigsqcup_{i=1}^{\ell} \mathcal{C}_{i}$ of $\left|\mathscr{L}_{n}(\mathcal{X})\right|$ into constructible subsets $\mathcal{C}_{i}$ and some $r_{1}, \ldots, r_{\ell}, j_{1}, \ldots, j_{\ell} \in \mathbb{N}$ such that

- for any $i \in\{1, \ldots, \ell\}$, we have $r_{i}-j_{i}=\operatorname{dim} \mathcal{X}$, and

$\circ$ for any $i \in\{1, \ldots, \ell\}$, any field extension $k^{\prime}$ of $k$ and any $\psi_{n} \in \mathscr{L}_{n}(\mathcal{X})\left(k^{\prime}\right)$ whose class in $\left|\mathscr{L}_{n}(\mathcal{X})\right|$ is contained in $\mathcal{C}_{i}$, we have

$$
\left(\theta_{n}^{n+1}\right)^{-1}\left(\psi_{n}\right) \cong \mathbb{A}_{k^{\prime}}^{r_{i}} \times_{k^{\prime}} B \mathbb{G}_{a}^{j_{i}}
$$

Proof. Fix $\xi_{n}$ : Spec $k^{\prime} \rightarrow \mathscr{L}_{n}(\mathcal{X})$ and let $\mathcal{Y}_{\xi_{n}}$ denote the fibre of the truncation map $\mathscr{L}_{n+1}(\mathcal{X}) \rightarrow$ $\mathscr{L}_{n}(\mathcal{X})$ over $\xi_{n}$. For any $\alpha$ : Spec $A \rightarrow$ Spec $k^{\prime}$, the $A$-valued points $\mathcal{Y}_{\xi_{n}}(A)$ are the category of lifts of $\xi_{n} \otimes_{k^{\prime}} A$ to $\mathscr{L}_{n+1}(\mathcal{X})$. For all $m \geq 0$, let $\mathcal{X}_{m}=\mathcal{X} \otimes_{k} k[t] /\left(t^{m+1}\right)$ and let $\alpha_{m}$ : Spec $A[t] /\left(t^{m+1}\right) \rightarrow$ Spec $k^{\prime}[t] /\left(t^{m+1}\right)$ be the map induced by $\alpha$; for $m \leq n$, let $\varphi_{m}$ : Spec $k^{\prime}[t] /\left(t^{m+1}\right) \rightarrow \mathcal{X}_{m}$ denote the map induced by $\xi_{n}$. We then obtain a cartesian diagram

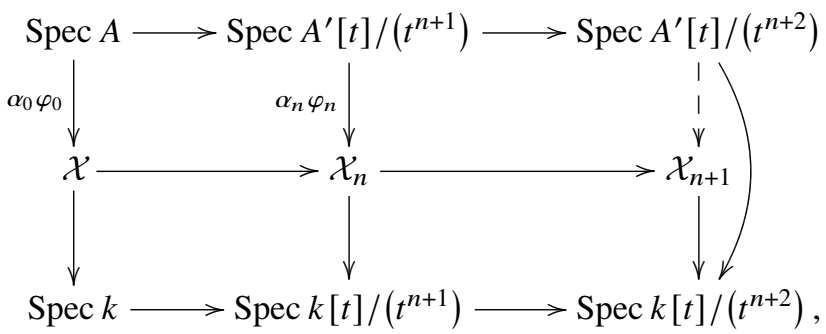

where the curved arrow is the structure map. Let $\mathcal{J}_{n}$ denote the ideal sheaf of Spec $k^{\prime}[t] /\left(t^{n+1}\right) \rightarrow$ Spec $k^{\prime}[t] /\left(t^{n+2}\right)$ considered as a $k^{\prime}$-module. By [Ols06, Theorem 1.5] and the fact that $\mathcal{X}_{n+1}$ and $A[t] /\left(t^{n+2}\right)$ are flat over $k[t] /\left(t^{n+2}\right)$, the obstruction to the existence of a dotted arrow in this diagram lives in

$$
\operatorname{Ext}^{1}\left(L\left(\alpha_{0} \varphi_{0}\right)^{*} L_{\mathcal{X} / k}, \alpha_{0}^{*} \mathcal{J}_{n}\right)=\operatorname{Ext}^{1}\left(L \varphi_{0}^{*} L_{\mathcal{X} / k}, \mathcal{O}_{k^{\prime}}\right) \otimes_{k^{\prime}} \mathcal{J}_{n} \otimes_{k^{\prime}} A
$$

We will show that this group vanishes, and so by [Ols06, Theorem 1.5], the objects (resp., automorphisms) of $\mathcal{Y}_{\xi_{n}}(A)$ are parametrised by $\operatorname{Ext}^{n}\left(L \varphi_{0}^{*} L_{\mathcal{X} / k}, \mathcal{O}_{k^{\prime}}\right) \otimes_{k^{\prime}} \mathcal{J}_{n} \otimes_{k^{\prime}} A$ where $n=0$ (resp., $\left.n=-1\right)$. In particular, if $V$ (resp., $G$ ) denotes the affine space (resp., algebraic vector group) over $k^{\prime}$ associated to the vector space $\operatorname{Ext}^{n}\left(L \varphi_{0}^{*} L_{\mathcal{X} / k}, \mathcal{O}_{k^{\prime}}\right) \otimes_{k^{\prime}} \mathcal{J}_{n}$ with $n=0$ (resp., $n=-1$ ), then we have

$$
\mathcal{Y}_{\xi_{n}} \cong V \times_{k^{\prime}} G \cong \mathbb{A}^{r\left(\xi_{n}\right)} \times B \mathbb{G}_{a}^{j\left(\xi_{n}\right)},
$$

where $r\left(\xi_{n}\right)=\operatorname{dim} \operatorname{Ext}^{0}\left(L \varphi_{0}^{*} L_{\mathcal{X} / k}, \mathcal{O}_{k^{\prime}}\right)$ and $j\left(\xi_{n}\right)=\operatorname{dim}_{\operatorname{Ext}^{1}}\left(L \varphi_{0}^{*} L_{\mathcal{X} / k}, \mathcal{O}_{k^{\prime}}\right)$. Note that this implies

$$
e\left(\mathcal{Y}_{\xi_{n}}\right)=\mathbb{L}^{r\left(\xi_{n}\right)-j\left(\xi_{n}\right)} .
$$


Therefore, to finish the proof of the theorem, it suffices to show that

$$
\operatorname{Ext}^{1}\left(L \varphi_{0}^{*} L_{\mathcal{X} / k}, \mathcal{O}_{k^{\prime}}\right)=0, \quad r\left(\xi_{n}\right)-j\left(\xi_{n}\right)=\operatorname{dim} \mathcal{X}
$$

and that the locus of $\varphi_{0} \in|\mathcal{X}|$ where $r\left(\xi_{n}\right)$ is constant is given by a constructible set. Since these remaining statements depend only on the dimension of the Ext-groups over $k^{\prime}$, it suffices to replace $k^{\prime}$ with an extension field, and hence we can assume $k^{\prime}$ is algebraically closed.

Let $\rho: \widetilde{X} \rightarrow \mathcal{X}$ be a smooth cover. Since $k^{\prime}$ is algebraically closed, we may fix a lift $\phi_{0}:$ Spec $k^{\prime} \rightarrow \widetilde{X}$ of $\varphi_{0}$. Since $\widetilde{X}$ and $\rho$ are smooth, we have an exact triangle

$$
p^{*} L_{\mathcal{X} / k} \rightarrow \Omega_{\widetilde{X} / k}^{1} \rightarrow \Omega_{\widetilde{X} / \mathcal{X}}^{1}
$$

from which we obtain an exact triangle

$$
\Gamma\left(\phi_{0}^{*} T_{\widetilde{X} / \mathcal{X}}\right) \rightarrow \Gamma\left(\phi_{0}^{*} T_{\widetilde{X} / k}\right) \rightarrow \operatorname{RHom}\left(L \varphi_{0}^{*} L_{\mathcal{X} / k}, \mathcal{O}_{k^{\prime}}\right)
$$

In particular, $\operatorname{Ext}^{n}\left(L \varphi_{0}^{*} L_{\mathcal{X} / k}, \mathcal{O}_{k^{\prime}}\right)=0$ for $n \neq 0,-1$, and there is an exact sequence

$$
0 \rightarrow \operatorname{Ext}^{-1}\left(L \varphi_{0}^{*} L_{\mathcal{X} / k}, \mathcal{O}_{k^{\prime}}\right) \rightarrow \Gamma\left(\phi_{0}^{*} T_{\widetilde{X} / \mathcal{X}}\right) \rightarrow \Gamma\left(\phi_{0}^{*} T_{\widetilde{X} / k}\right) \rightarrow \operatorname{Ext}^{0}\left(L \varphi_{0}^{*} L_{\mathcal{X} / k}, \mathcal{O}_{k^{\prime}}\right) \rightarrow 0
$$

Thus,

$$
r\left(\xi_{n}\right)-j\left(\xi_{n}\right)=\operatorname{dim} \Gamma\left(\phi_{0}^{*} T_{\widetilde{X} / k}\right)-\operatorname{dim} \Gamma\left(\phi_{0}^{*} T_{\widetilde{X} / \mathcal{X}}\right)=\operatorname{dim} \mathcal{X}
$$

thereby establishing equation (1). Finally, note from formula (2) that the cokernel of $\Gamma\left(\phi_{0}^{*} T_{\widetilde{X} / \mathcal{X}}\right) \rightarrow$ $\Gamma\left(\phi_{0}^{*} T_{\widetilde{X} / k}\right)$ depends only on $\varphi_{0}$ and not on the choice of lift $\phi_{0}$. So the locus of $\varphi_{0} \in|\mathcal{X}|$ where $r\left(\xi_{n}\right)$ is constant is the image under $\rho$ of the locus of $\phi_{0} \in|\widetilde{X}|$ where the dimension is constant. By Chevalley's theorem for Artin stacks [HR17, Theorem 5.2], it is therefore enough to show that the locus of such $\phi_{0}$ is constructible. This follows by applying [Sta21, Lemma 0BDI] to the 2-term complex $T_{\widetilde{X} / \mathcal{X}} \rightarrow T_{\widetilde{X} / k}$ and using the fact that $|\widetilde{X}|$ is Noetherian, so that all locally constructible sets are constructible.

Lemma 3.35. Let $\mathcal{Y}, \mathcal{Z}$ and $\mathcal{F}$ be finite-type Artin stacks over $k$, let $\mathcal{Y} \rightarrow \mathcal{Z}$ be a $k$-morphism and assume that for any field extensions $k^{\prime}$ of $k$ and any $k$-morphism $\operatorname{Spec}\left(k^{\prime}\right) \rightarrow \mathcal{Z}$, there exists a $k^{\prime}$-isomorphism

$$
\left(\mathcal{Y} \times_{\mathcal{Z}} \operatorname{Spec}\left(k^{\prime}\right)\right)_{\text {red }} \cong\left(\mathcal{F} \times_{\operatorname{Spec}(k)} \operatorname{Spec}\left(k^{\prime}\right)\right)_{\text {red }} .
$$

Then

$$
\mathrm{e}(\mathcal{Y})=\mathrm{e}(\mathcal{F}) \mathrm{e}(\mathcal{Z}) \in K_{0}\left(\operatorname{Stack}_{k}\right)
$$

Proof. Because $\mathcal{Z}$ can be stratified by quotient stacks [Kre99, Proposition 3.5.9], we may assume that $\mathcal{Z}=[Z / G]$ for some finite-type scheme $Z$ over $k$ with an action by a general linear group $G$ over $k$. Let $\mathcal{Y}^{\prime}=\mathcal{Y} \times_{\mathcal{Z}} Z$. Because $Z \rightarrow \mathcal{Z}$ and $\mathcal{Y}^{\prime} \rightarrow \mathcal{Y}$ are $G$-torsors and $G$ is a special group, [Eke09, Proposition 1.1(ii)] gives

$$
\begin{aligned}
\mathrm{e}(Z) & =\mathrm{e}(G) \mathrm{e}(\mathcal{Z}) \\
\mathrm{e}\left(\mathcal{Y}^{\prime}\right) & =\mathrm{e}(G) \mathrm{e}(\mathcal{Y})
\end{aligned}
$$


By the hypotheses on $\mathcal{Y} \rightarrow \mathcal{Z}$, Proposition 2.8 implies that $\mathcal{Y}^{\prime} \rightarrow Z$ is a piecewise trivial fibration with fibre $\mathcal{F}$, so in particular,

$$
\mathrm{e}\left(\mathcal{Y}^{\prime}\right)=\mathrm{e}(\mathcal{F}) \mathrm{e}(Z)
$$

Thus,

$$
\mathrm{e}(G) \mathrm{e}(\mathcal{Y})=\mathrm{e}(G) \mathrm{e}(\mathcal{F}) \mathrm{e}(\mathcal{Z})
$$

Because $G$ is a special group, e $(G)^{-1} \in K_{0}\left(\mathbf{S t a c k}_{k}\right)$, so we are done.

We may now prove Theorem 3.33 .

Proof of Theorem 3.33. By definition, there exist some $n_{0} \in \mathbb{N}$ and some constructible subset $\mathcal{C}_{n_{0}} \subset$ $\left|\mathscr{L}_{n_{0}}(\mathcal{X})\right|$ such that $\mathcal{C}=\left(\theta_{n_{0}}\right)^{-1}\left(\mathcal{C}_{n_{0}}\right)$. Because $\mathcal{X}$ is smooth, infinitesimal lifting implies that the truncation maps $\theta_{n}:|\mathscr{L}(\mathcal{X})| \rightarrow\left|\mathscr{L}_{n}(\mathcal{X})\right|$ are all surjective, so

$$
\theta_{n}(\mathcal{C})= \begin{cases}\left(\theta_{n_{0}}^{n}\right)^{-1}\left(\mathcal{C}_{n_{0}}\right), & n \geq n_{0}, \\ \theta_{n}^{n_{0}}\left(\mathcal{C}_{n_{0}}\right), & n<n_{0}\end{cases}
$$

Thus all $\theta_{n}(\mathcal{C})$ are constructible - immediately for $n \geq n_{0}$, and by Chevalley's theorem for Artin stacks [HR17, Theorem 5.2] for $n<n_{0}$.

The remainder of the theorem then follows from the fact that Lemma 3.34 and Lemma 3.35 imply that for any $n \geq n_{0}$,

$$
\mathrm{e}\left(\theta_{n}(\mathcal{C})\right)=\left(\theta_{n_{0}}^{n}\right)^{-1}\left(\mathcal{C}_{n_{0}}\right)=\mathrm{e}\left(\mathcal{C}_{n_{0}}\right) \mathbb{L}^{\left(n-n_{0}\right) \operatorname{dim} \mathcal{X}}
$$

\section{Fibres of the map of arcs}

Our goal in this section is to give a combinatorial characterisation of the fibres of $\mathscr{L}(\pi): \mathscr{L}(\mathcal{X}) \rightarrow$ $\mathscr{L}(X)$, where $\mathcal{X}$ is a fantastack and $\pi: \mathcal{X} \rightarrow X$ is its good moduli space map (see Theorem 4.9). We accomplish this goal by first defining the tropicalisation of arcs both for toric varieties and for toric stacks.

\subsection{Tropicalising arcs of toric stacks}

Given a toric variety $X=\operatorname{Spec}(k[P])$, a $k$-algebra $R$ and an $\operatorname{arc} \varphi \in \mathscr{L}(\widetilde{X})(R)$, we denote by $\varphi^{*}(p)$ the image of $p$ under $P \rightarrow k[P] \rightarrow R \llbracket t \rrbracket$, where the latter map is the pullback corresponding to $\varphi$.

Definition 4.1. If $\sigma$ is a pointed rational cone on a finite-rank lattice $N$ and $k^{\prime}$ is a field extension of $k$, we define the tropicalisation map

$$
\text { trop : } \mathscr{L}\left(X_{\sigma}\right)\left(k^{\prime}\right) \rightarrow \operatorname{Hom}\left(\sigma^{\vee} \cap N^{*}, \mathbb{N} \cup\{\infty\}\right)
$$

by $\operatorname{trop}(\varphi)(p):=\operatorname{ord}_{t} \varphi^{*}(p)$, where $\operatorname{ord}_{t}$ denotes the order of vanishing at $t$.

More generally, if $(\sigma, v: \widetilde{N} \rightarrow N)$ is a stacky fan with $\sigma$ a pointed cone and $\mathcal{X}:=\mathcal{X}_{\sigma, v}:=\left[X_{\sigma} / G_{v}\right]$ is the corresponding toric stack, then we define the tropicalisation map on isomorphism classes of arcs

$$
\operatorname{trop}: \overline{\mathscr{L}(\mathcal{X})}\left(k^{\prime}\right) \rightarrow \operatorname{Hom}\left(\sigma^{\vee} \cap \widetilde{N}^{*}, \mathbb{N} \cup\{\infty\}\right)
$$


as follows. If $\psi \in \mathscr{L}(\mathcal{X})\left(k^{\prime}\right)$, then fix a finite field extension $k^{\prime \prime}$ of $k^{\prime}$ and a lift $\widetilde{\psi} \in \mathscr{L}\left(X_{\sigma}\right)\left(k^{\prime \prime}\right)$ of $\psi$. We define $\operatorname{trop}(\psi):=\operatorname{trop}(\widetilde{\psi})$. We show in Lemma 4.4 that this is well defined.

Remark 4.2. Note that we have a natural inclusion

$$
\sigma \cap N=\operatorname{Hom}\left(\sigma^{\vee} \cap N^{*}, \mathbb{N}\right) \subset \operatorname{Hom}\left(\sigma^{\vee} \cap N^{*}, \mathbb{N} \cup\{\infty\}\right)
$$

Lemma 4.3. Let $\Omega$ be any field of characteristic 0 and let $G=\mathbb{G}_{m}^{r} \times \prod_{i=1}^{N} \mu_{n_{i}}$. Then every G-torsor over $\operatorname{Spec}(\Omega[[t]])$ is isomorphic to the pullback of a $G$-torsor over $\operatorname{Spec}(\Omega)$.

Proof. Let $q: \operatorname{Spec}(\Omega[[t]]) \rightarrow \operatorname{Spec}(\Omega)$ denote the structure map. Since $G$ is an étale group scheme, $G$-torsors on any $\Omega$-scheme $Y$ are classified up to isomorphism by $H_{e t}^{1}(Y, G)=H_{e t}^{1}\left(Y, \mathbb{G}_{m}\right)^{\oplus r} \oplus$ $\bigoplus_{i=1}^{N} H_{e t}^{1}\left(Y, \mu_{n_{i}}\right)$. In particular, it suffices to show that the pullback map $q^{*}: H_{e t}^{1}(\operatorname{Spec}(\Omega), G) \rightarrow$ $H_{e t}^{1}(\operatorname{Spec}(\Omega[[t]]), G)$ is an isomorphism when $G$ is either $\mathbb{G}_{m}$ or $\mu_{n}$.

We first handle the case $G=\mathbb{G}_{m}$. Since $H_{e t}^{1}\left(Y, \mathbb{G}_{m}\right)=\operatorname{Pic}(Y)$ and since both $\operatorname{Pic}(\operatorname{Spec}(\Omega))$ and $\operatorname{Pic}(\operatorname{Spec}(\Omega[[t]]))$ are trivial, we see that $q^{*}$ is an isomorphism.

We next handle the case $G=\mu_{n}$. From the Kummer sequence

$$
1 \rightarrow \mu_{n} \rightarrow \mathbb{G}_{m} \stackrel{\times n}{\longrightarrow} \mathbb{G}_{m} \rightarrow 1
$$

we see that if $Y$ is any $\Omega$-scheme with trivial Picard group, we have

$$
H_{e t}^{1}\left(Y, \mu_{n}\right)=\mathcal{O}_{Y}(Y)^{*} /\left(\mathcal{O}_{Y}(Y)^{*}\right)^{n}
$$

(see, for example, [Mil80, p. 125]). So it remains to show that $k^{*} /\left(k^{*}\right)^{n} \rightarrow k[[t]]^{*} /\left(k[[t]]^{*}\right)^{n}$ is an isomorphism. Since every element $f(t) \in k[[t]]^{*}$ can be written uniquely as $a g(t)$ with $a \in k^{*}$ and $g(t) \in k[[t]]^{*}$ with $g(t)-1 \in t k[[t]]$, it is enough to prove that such $g(t)$ are in $\left(k[[t]]^{*}\right)^{n}$. This follows immediately from Hensel's lemma: the polynomial $P(x)=x^{n}-g(t) \in k[[t]][x]$ has a root, since $P(1)=0 \bmod t$ and $P^{\prime}(1) \neq 0 \bmod t$.

Lemma 4.4. With notation as in Definition 4.1, such a lift $\widetilde{\psi}$ exists, and $\operatorname{trop}(\psi)$ is independent of both $k^{\prime \prime}$ and $\widetilde{\psi}$.

Proof. For ease of notation, write $F:=\sigma^{\vee} \cap \widetilde{N}^{*}$ and $G:=G_{v}=\operatorname{Spec}(k[A])$, where $A$ is a finitely generated abelian group. Note that the $G$-action on $X_{\sigma}$ corresponds to a monoid map $\eta: F \rightarrow A$. The $\operatorname{arc} \psi$ corresponds to a $G$-torsor $Q \rightarrow \operatorname{Spec}\left(k^{\prime} \llbracket t \rrbracket\right)$ and $G$-equivariant map $Q \rightarrow X_{\sigma}$. By Lemma 4.3, $Q$ is isomorphic to the pullback of a $G$-torsor over $\operatorname{Spec}\left(k^{\prime}\right)$, which can be itself be trivialised after a finite field extension $k^{\prime \prime}$. Thus, after base change to $k^{\prime \prime} \otimes_{k^{\prime}} k^{\prime} \llbracket t \rrbracket \simeq k^{\prime \prime} \llbracket t \rrbracket$, we obtain a trivialisation of $Q$ and hence a lift $\widetilde{\psi}$.

Next, it is clear that if $\widetilde{\psi} \in \mathscr{L}\left(X_{\sigma}\right)\left(k^{\prime \prime}\right)$ is a lift of $\psi$ and $k^{\prime \prime \prime}$ is a finite field extension of $k^{\prime \prime}$, then $\operatorname{trop}(\widetilde{\psi})=\operatorname{trop}\left(\widetilde{\psi} \otimes_{k^{\prime \prime}} k^{\prime \prime \prime}\right)$. So it suffices to show that if $\widetilde{\psi}_{1}, \widetilde{\psi}_{2} \in \mathscr{L}\left(X_{\sigma}\right)\left(k^{\prime \prime}\right)$ are both lifts of $\psi$, then trop $\left(\widetilde{\psi}_{1}\right)=\operatorname{trop}\left(\widetilde{\psi}_{2}\right)$. In this case, there exists $g \in G\left(k^{\prime \prime} \llbracket t \rrbracket\right)$ such that $g \cdot \widetilde{\psi}_{1}=\widetilde{\psi}_{2}$. Letting $g^{*}(a)$ denote the pullback of $a \in A$ under the map $g^{*}: k^{\prime \prime}[A] \rightarrow k^{\prime \prime} \llbracket t \rrbracket^{*}$, we therefore have

$$
g^{*}(\eta(f)) \psi_{1}^{*}(f)=\psi_{2}^{*}(f)
$$

Since $g^{*}(\eta(f))$ is a unit, the power series $\psi_{1}^{*}(f)$ and $\psi_{2}^{*}(f)$ have the same $t$-order of vanishing - that is, $\operatorname{trop}\left(\widetilde{\psi}_{1}\right)=\operatorname{trop}\left(\widetilde{\psi}_{2}\right)$.

We record some basic properties of trop that will be useful later on. 
Definition 4.5. Let $(\sigma, v: \widetilde{N} \rightarrow N)$ be a stacky fan with $\sigma$ a pointed cone. For any $w \in \sigma \cap \widetilde{N} \subset$ $\operatorname{Hom}\left(\sigma^{\vee} \cap \widetilde{N}^{*}, \mathbb{N} \cup\{\infty\}\right)$, let

$$
\operatorname{trop}^{-1}(w)=\left\{\psi \in \overline{\mathscr{L}\left(\mathcal{X}_{\sigma, v}\right)}\left(k^{\prime}\right) \mid k^{\prime} \text { is a field extension of } k \text { and } \operatorname{trop}(\psi)=w\right\} \subset\left|\mathscr{L}\left(\mathcal{X}_{\sigma, v}\right)\right|,
$$

where the arcs are taken up to equivalence.

Remark 4.6. Let $\sigma$ be a pointed rational cone on a finite-rank lattice $N$ and let $P=\sigma^{\vee} \cap N^{*}$ so that $X_{\sigma}=\operatorname{Spec}(k[P])$. For any $p \in P$, let $\chi^{p} \in k[P]$ be the corresponding monomial. If $p_{1}, \ldots, p_{s}$ are generators for $P$, then for every $w \in \sigma \cap N$, we see

$$
\operatorname{trop}^{-1}(w)=\bigcap_{i=1}^{s} \operatorname{ord}_{\chi^{p_{i}}}^{-1}\left(\left\langle w, p_{i}\right\rangle\right),
$$

and hence $\operatorname{trop}^{-1}(w) \subset \mathscr{L}\left(X_{\sigma}\right)$ is a cylinder.

Lemma 4.7. Let $\sigma$ (resp., $\widetilde{\sigma})$ be a pointed rational cone on a finite-rank lattice $N$ (resp., $\widetilde{N})$. If $\rho: X_{\widetilde{\sigma}} \rightarrow X_{\sigma}$ is a toric morphism and $\beta: \widetilde{\sigma} \cap \widetilde{N} \rightarrow \sigma \cap N$ is the induced map, then

1. for every field extension $k^{\prime}$ of $k$ and every $\operatorname{arc} \psi \in \mathscr{L}\left(X_{\widetilde{\sigma}}\right)\left(k^{\prime}\right)$, if $\operatorname{trop}(\psi) \in \widetilde{\sigma}^{\vee} \cap \widetilde{N}^{*}$, then

$$
\operatorname{trop}(\mathscr{L}(\rho)(\psi))=\beta(\operatorname{trop}(\psi)) ;
$$

2. iffor all $f \in \widetilde{\sigma}^{\vee} \cap \widetilde{N}^{*}$ there exists $f^{\prime} \in \widetilde{\sigma}^{\vee} \cap \widetilde{N}^{*}$ such that $f+f^{\prime}$ lies in the image of $\sigma^{\vee} \cap N^{*}$, then

$$
\mathscr{L}(\rho)^{-1}\left(\operatorname{trop}^{-1}(w)\right)=\bigcup_{\widetilde{w} \in \beta^{-1}(w)} \operatorname{trop}^{-1}(\widetilde{w}) .
$$

Proof. Let $\rho^{*}: \sigma^{\vee} \cap N^{*} \rightarrow \widetilde{\sigma}^{\vee} \cap \widetilde{N}^{*}$ denote the pullback map on monoids. First note that if $k^{\prime}$ is a field extension of $k, \psi \in \mathscr{L}\left(X_{\widetilde{\sigma}}\right)\left(k^{\prime}\right)$ and $p \in \sigma^{\vee} \cap N^{*}$, then

$$
\operatorname{trop}(\mathscr{L}(\rho)(\psi))(p)=\operatorname{ord}_{t}\left(\psi^{*} \rho^{*}(p)\right)=(\operatorname{trop}(\psi))\left(\rho^{*}(p)\right) .
$$

To prove part (1), let $\operatorname{trop}(\psi)=\widetilde{w} \in \widetilde{\sigma}^{\vee} \cap \widetilde{N}^{*}$. Then by these equalities, we see

$$
\operatorname{trop}(\mathscr{L}(\rho)(\psi))(p)=\left\langle\widetilde{w}, \rho^{*}(p)\right\rangle=\langle\beta(\widetilde{w}), p\rangle,
$$

so $\operatorname{trop}(\mathscr{L}(\rho)(\psi))=\beta(\widetilde{w})$.

Part (2) follows immediately from part (1), provided we can show that $\operatorname{trop}(\mathscr{L}(\rho)(\psi)) \in \sigma \cap N$ implies $\operatorname{trop}(\psi) \in \widetilde{\sigma} \cap \widetilde{N}$. Set $f \in \widetilde{\sigma}^{\vee} \cap \widetilde{N}^{*}$. By hypothesis, there exists $f^{\prime} \in \widetilde{\sigma}^{\vee} \cap \widetilde{N}^{*}$ such that $f+f^{\prime}=\rho^{*}(p)$ for some $p \sigma^{\vee} \cap N^{*}$. Then

$$
(\operatorname{trop}(\psi))(f)+(\operatorname{trop}(\psi))\left(f^{\prime}\right)=(\operatorname{trop}(\psi))\left(\rho^{*}(p)\right)=\operatorname{trop}(\mathscr{L}(\rho)(\psi))(p) \neq \infty .
$$

So $(\operatorname{trop}(\psi))(f) \neq \infty$ for all $f$, and hence $\operatorname{trop}(\psi) \in \widetilde{\sigma} \cap \widetilde{N}$.

Corollary 4.8. Let $\mathcal{X}=\mathcal{F}_{\sigma, v}$ be a fantastack and suppose the good moduli space map $\pi: \mathcal{X} \rightarrow X=X_{\sigma}$ is an isomorphism over the torus $T \subset X$. Let $k^{\prime}$ be a field extension of $k$ and $\varphi \in \mathscr{L}(X)\left(k^{\prime}\right)$ with $\operatorname{trop}(\varphi)=w \in \sigma \cap N$. If $\psi \in \mathscr{L}(\mathcal{X})\left(k^{\prime}\right)$ and $\mathscr{L}(\pi)(\psi)=\varphi$, then $\operatorname{trop}(\psi) \in \beta^{-1}(w)$.

Proof. We keep the notation listed in Notation 2.19 and let $\mathcal{X}=\left[\widetilde{X} / G_{v}\right]$. We know there exist a finite field extension $k^{\prime \prime}$ of $k^{\prime}$ and a lift $\tilde{\psi} \in \mathscr{L}(\widetilde{X})\left(k^{\prime \prime}\right)$ of $\psi$. By construction, $\operatorname{trop}(\psi)=\operatorname{trop}(\widetilde{\psi})$. From Proposition 2.14, we know the hypotheses of Lemma 4.7(2) are satisfied, so trop $(\widetilde{\psi}) \in \beta^{-1}(w)$. 


\subsection{Lifting arcs to a fantastack}

We can now state the main result of this section, which shows that for the good moduli space map $\pi: \mathcal{X} \rightarrow X$ of a fantastack, isomorphism classes of arcs in the fibres of $\mathscr{L}(\pi)$ are completely determined by their tropicalisations.

Theorem 4.9. Let $\mathcal{X}=\mathcal{F}_{\sigma, v}$ be a fantastack and assume the good moduli space map $\pi: \mathcal{X} \rightarrow X:=X_{\sigma}$ is an isomorphism over the torus $T \subset X$. With the notation listed in Notation 2.19 , let $k^{\prime}$ be a field extension of $k$ and $\varphi \in \mathscr{L}(X)\left(k^{\prime}\right)$ with $\operatorname{trop}(\varphi)=w \in \sigma \cap N$. Then

$$
\text { trop }: \overline{\mathscr{L}(\mathcal{X})}\left(k^{\prime}\right) \rightarrow \operatorname{Hom}(F, \mathbb{N} \cup\{\infty\})
$$

induces, by restriction, a bijection

$$
\overline{\left(\mathscr{L}(\pi)^{-1}(\varphi)\right)}\left(k^{\prime}\right) \rightarrow \beta^{-1}(w) .
$$

In particular, $\overline{\left(\mathscr{L}(\pi)^{-1}(\varphi)\right)}\left(k^{\prime}\right)$ is a finite set.

For the rest of this section, we fix the notation as in the statement of Theorem Theorem 4.9. By Corollary 4.8, we know that any $\psi \in \mathscr{L}(\mathcal{X})\left(k^{\prime}\right)$ with $(\mathscr{L}(\pi))(\psi)=\varphi$ must satisfy trop $(\psi) \in \beta^{-1}(w)$. We therefore have an induced map $\overline{\left(\mathscr{L}(\pi)^{-1}(\varphi)\right)}\left(k^{\prime}\right) \rightarrow \beta^{-1}(w)$. We show injectivity and surjectivity in Proposition 4.10 and Proposition 4.11, respectively. Note that the finiteness of $\overline{\left(\mathscr{L}(\pi)^{-1}(\varphi)\right)}\left(k^{\prime}\right)$ then follows from Proposition 2.15.

Proposition 4.10. The restriction of the map

$$
\operatorname{trop}: \overline{\mathscr{L}(\mathcal{X})}\left(k^{\prime}\right) \rightarrow \operatorname{Hom}(F, \mathbb{N} \cup\{\infty\})
$$

to $\overline{\left(\mathscr{L}(\pi)^{-1}(\varphi)\right)}\left(k^{\prime}\right)$ is injective.

Proof. Let $\psi_{1}, \psi_{2} \in \mathscr{L}(\mathcal{X})\left(k^{\prime}\right)$ correspond to $G$-torsors $Q_{1}, Q_{2} \rightarrow \operatorname{Spec}\left(k^{\prime} \llbracket t \rrbracket\right)$ and $G$-equivariant maps $\gamma_{1}: Q_{1} \rightarrow \widetilde{X}$ and $\gamma_{2}: Q_{2} \rightarrow \widetilde{X}$, respectively, and assume that $\mathscr{L}(\pi)\left(\psi_{1}\right)=\mathscr{L}(\pi)\left(\psi_{2}\right)=\varphi$ and $\operatorname{trop}\left(\psi_{1}\right)=\operatorname{trop}\left(\psi_{2}\right)$. We need to prove that $\psi_{1}$ and $\psi_{2}$ are isomorphic - that is, that there exists an isomorphism of $G$-torsors $\alpha: Q_{1} \rightarrow Q_{2}$ such that $\gamma_{1}=\gamma_{2} \circ \alpha$. In fact, we prove the stronger statement that there exists a unique such $\alpha$.

To prove this stronger statement, by descent, it is enough to show the existence of a unique such $\alpha$ étale locally on $k^{\prime} \llbracket t \rrbracket$. By Lemma 4.3, the $Q_{i}$ are isomorphic to pullbacks of torsors over $k^{\prime}$, which can themselves be trivialised after base change to a finite field extension $k^{\prime \prime}$ of $k^{\prime}$. Since $k^{\prime \prime} \otimes_{k^{\prime}} k^{\prime} \llbracket t \rrbracket \cong$ $k^{\prime \prime} \llbracket t \rrbracket$, after replacing $k^{\prime}$ by $k^{\prime \prime}$, we may therefore assume that the $Q_{i}$ are trivial $G$-torsors. Since $\operatorname{trop}\left(\psi_{i}\right)$ depends only on the isomorphism class of $\psi_{i}$, we may further assume $Q_{1}=Q_{2}=G \otimes_{k} k^{\prime} \llbracket t \rrbracket$.

Next, the identity section of the $G$-torsor $Q_{i}$ then yields a lift $\widetilde{\psi}_{i} \in \mathscr{L}(\widetilde{X})\left(k^{\prime}\right)$ of $\psi_{i}$. Then the map $\gamma_{i}: \operatorname{Spec}\left(k^{\prime} \llbracket t \rrbracket[A]\right)=Q_{i} \rightarrow \widetilde{X}=\operatorname{Spec}(k[F])$ satisfies

$$
\gamma_{i}^{*}(f)=\widetilde{\psi}_{i}^{*}(f) u^{\bar{f}} \in k^{\prime} \llbracket t \rrbracket[A],
$$

where $u^{\bar{f}} \in k[A]$ is the monomial indexed by the image $\bar{f}$ of $f$ in $A$. Since $\operatorname{trop}\left(\widetilde{\psi}_{i}\right)=\operatorname{trop}\left(\psi_{i}\right)$, we see $\operatorname{trop}\left(\widetilde{\psi}_{1}\right)=\operatorname{trop}\left(\widetilde{\psi}_{2}\right) \in \widetilde{\sigma} \cap \widetilde{N}$. Thus, for all $f \in F$, the power series $\widetilde{\psi}_{1}^{*}(f)$ and $\widetilde{\psi}_{2}^{*}(f)$ are nonzero and have the same $t$-order of vanishing. It follows that there is a unique unit $g^{(f)} \in k^{\prime} \llbracket t \rrbracket$ such that

$$
\psi_{1}^{*}(f)=g^{(f)} \psi_{2}^{*}(f) .
$$

Since $\mathscr{L}(\pi)\left(\widetilde{\psi}_{1}\right)=\mathscr{L}(\pi)\left(\widetilde{\psi}_{2}\right)$, we see that $\widetilde{\psi}_{1}^{*}(p)=\widetilde{\psi}_{2}^{*}(p)$, and so $g^{(p)}=1$ for all $p \in P$. 
Thus the semigroup homomorphism $F \rightarrow k^{\prime} \llbracket t \rrbracket^{\times}: f \mapsto g^{(f)}$ induces a group homomorphism $A \rightarrow k^{\prime} \llbracket t \rrbracket^{\times}$, which corresponds to an element $g \in G\left(k^{\prime} \llbracket t \rrbracket\right)$ and hence an automorphism $\alpha$ of the $G$-torsor $G \otimes_{k} k^{\prime} \llbracket t \rrbracket$. By construction, $\widetilde{\psi_{1}}=\widetilde{\psi_{2}} \circ \alpha$, and so $\gamma_{1}=\gamma_{2} \circ \alpha$. Moreover, the uniqueness of $\alpha$ follows from the uniqueness of each $g^{(f)}$.

We now complete the proof of Theorem 4.9 by showing the surjectivity of the map $\overline{\left(\mathscr{L}(\pi)^{-1}(\varphi)\right)}\left(k^{\prime}\right) \rightarrow \beta^{-1}(w)$.

Proposition 4.11. The image of $\overline{\left(\mathscr{L}(\pi)^{-1}(\varphi)\right)}\left(k^{\prime}\right)$ under the map

$$
\operatorname{trop}: \overline{\mathscr{L}(\mathcal{X})}\left(k^{\prime}\right) \rightarrow \operatorname{Hom}(F, \mathbb{N} \cup\{\infty\})
$$

is equal to $\beta^{-1}(w)$.

Proof. Recall that in Corollary 4.8, we proved that the image of $\overline{\left(\mathscr{L}(\pi)^{-1}(\varphi)\right)}\left(k^{\prime}\right)$ under trop is contained in $\beta^{-1}(w)$.

Set $\widetilde{w} \in \beta^{-1}(w)$. We will construct an $\operatorname{arc} \psi \in \mathscr{L}(\mathcal{X})\left(k^{\prime}\right)$ satisfying $\operatorname{trop}(\psi)=\widetilde{w}$ and $\mathscr{L}(\pi)(\psi)=\varphi$. Let $\eta: \operatorname{Spec}\left(k^{\prime}\left(t_{D}\right) \rightarrow X\right.$ be the generic point of $\varphi-$ that is, $\eta$ is the composition $\operatorname{Spec}\left(k^{\prime}(t)\right) \rightarrow$ $\operatorname{Spec}\left(k^{\prime} \llbracket t \rrbracket\right) \stackrel{\varphi}{\rightarrow} X$. Since $w \in \sigma \cap N$, we see that $\eta$ factors through $T \hookrightarrow X$. Thus $\eta$ is given by a group homomorphism $M=P^{\mathrm{gp}} \rightarrow k^{\prime}(t)^{\times}$.

Given our inclusion $M \hookrightarrow \widetilde{M}$, we can choose a $\mathbb{Z}$-basis $f_{1}, \ldots, f_{r}$ for $\widetilde{M}=F^{\mathrm{gp}}$ and $m_{1}, \ldots, m_{d} \in \mathbb{Z}_{>0}$ such that $m_{1} f_{1}, \ldots, m_{d} f_{d}$ is a $\mathbb{Z}$-basis for $M$. Since $\operatorname{trop}(\varphi)=w$, the image of $m_{i} f_{i}$ under $M \rightarrow k^{\prime}(t)^{\times}$ has $t$-order of vanishing $\left\langle w, m_{i} f_{i}\right\rangle$, so it can be written as $t^{\left\langle w, m_{i} f_{i}\right\rangle} g_{i}$ with $g_{i} \in k^{\prime} \llbracket t \rrbracket^{\times}$.

Now set $R$ to be the $k^{\prime} \llbracket t \rrbracket$-algebra

$$
R=k^{\prime} \llbracket t \rrbracket\left[x_{1}, \ldots, x_{d}, x_{d+1}^{ \pm 1}, \ldots, x_{r}^{ \pm 1}\right] /\left(x_{1}^{m_{1}}-g_{1}, \ldots, x_{d}^{m_{d}}-g_{d}\right)
$$

and give $\operatorname{Spec}(R)$ the $G$-action obtained by letting $x_{i}$ have grading $\bar{f}_{i}$, where $\bar{f}_{i}$ is the image of $f_{i}$ in $A$. Since each $g_{i}$ is a unit in $k^{\prime} \llbracket t \rrbracket$, we see that $\operatorname{Spec}(R) \rightarrow \operatorname{Spec}\left(k^{\prime} \llbracket t \rrbracket\right)$ is a $G$-torsor.

Let $\psi \in \mathscr{L}(\mathcal{X})\left(k^{\prime}\right)$ be the arc corresponding to the $G$-torsor $\operatorname{Spec}(R)$ and the $G$-equivariant map $\operatorname{Spec}(R) \rightarrow \widetilde{X}=\operatorname{Spec}(k[F])$ defined by

$$
F \rightarrow R: f \mapsto t^{\langle\widetilde{w}, f\rangle} \prod_{i=1}^{r} x_{i}^{c_{i}},
$$

for $f=\sum_{i=1}^{r} c_{i} f_{i}$ and $c_{1}, \ldots, c_{r} \in \mathbb{Z}$. Note that this map is well defined, since $\langle\widetilde{w}, f\rangle \geq 0$ when $f \in F$, and each $x_{i}$ is a unit in $R$ as a consequence of the fact that each $g_{i}$ is a unit in $k^{\prime} \llbracket t \rrbracket$. Note further that since the $x_{i}$ are units, the map sends $f$ to an element whose $t$-order of vanishing is $\langle\widetilde{w}, f\rangle$. As a result, any lift $\widetilde{\psi} \in \mathscr{L}(\widetilde{X})\left(k^{\prime \prime}\right)$ obtained from a trivialisation of the $G$-torsor after base change to $k^{\prime \prime}$, satisfies $\operatorname{trop}(\psi)=\operatorname{trop}(\widetilde{\psi})=\widetilde{w}$.

To finish the proof, we must show $\mathscr{L}(\pi)(\psi)=\varphi-$ that is, $\pi \circ \psi=\varphi$. Since $X$ is separated, it is enough to prove equality after precomposing by the generic point $\xi: \operatorname{Spec}\left(k^{\prime}(t)\right) \rightarrow \operatorname{Spec}\left(k^{\prime} \llbracket t \rrbracket\right)$. But this follows from the facts that $\eta=\varphi \circ \xi$ and that for any $i \in\{1, \ldots, d\}$, the image of $m_{i} f_{i}$ under the pullback of $\operatorname{Spec}(R) \rightarrow \widetilde{X} \stackrel{\widetilde{\pi}}{\rightarrow} X$ is equal to

$$
t^{\left\langle\widetilde{w}, m_{i} f_{i}\right\rangle} x_{i}^{m_{i}}=t^{\left\langle w, m_{i} f_{i}\right\rangle} g_{i}
$$




\section{Fibres of the maps of jets}

Throughout this section set $d \in \mathbb{N}$, let $N \cong \mathbb{Z}^{d}$ be a lattice, let $T=\operatorname{Spec}\left(k\left[N^{*}\right]\right)$ be the algebraic torus with cocharacter lattice $N$, let $\sigma$ be a pointed rational cone in $N_{\mathbb{R}}$, let $X$ be the affine $T$-toric variety associated to $\sigma$, let $\mathcal{X}$ be the canonical stack over $X$ and let $\pi: \mathcal{X} \rightarrow X$ be the canonical map.

In this section we will control the fibres of the maps

$$
\mathscr{L}_{n}(\pi): \mathscr{L}_{n}(\mathcal{X}) \rightarrow \mathscr{L}_{n}(X)
$$

in the case where $\sigma$ is $d$-dimensional. In particular, we will prove the following:

Theorem 5.1. Suppose that $\sigma$ is d-dimensional, and set $w \in \sigma \cap N$. Then there exist $n_{w} \in \mathbb{N}$ and $\Theta_{w} \in K_{0}\left(\mathbf{S t a c k}_{k}\right)$ and a sequence of finite-type Artin stacks $\left\{\mathcal{F}_{n}\right\}_{n \geq n_{w}}$ over $k$ such that

1. for each $n \geq n_{w}$,

$$
\mathrm{e}\left(\mathcal{F}_{n}\right)=\Theta_{w} \in K_{0}\left(\mathbf{S t a c k}_{k}\right)
$$

and

2. for each field extension $k^{\prime}$ of $k$, each $\operatorname{arc} \varphi \in \mathscr{L}(X)\left(k^{\prime}\right)$ with $\operatorname{trop}(\varphi)=w$ and each $n \geq n_{w}$, we have

$$
\mathscr{L}_{n}(\pi)^{-1}\left(\theta_{n}(\varphi)\right)_{\mathrm{red}} \cong \mathcal{F}_{n} \otimes_{k} k^{\prime}
$$

For the remainder of this section, we will assume that $\sigma$ is $d$-dimensional, and we will use the notation listed in Notation 2.19.

\subsection{Algebraic groups and jets}

We begin by introducing some algebraic groups, which in Proposition 5.15 will be used to express the fibres of each $\mathscr{L}_{n}(\pi)$.

Remark 5.2. In what follows, for each $k$-algebra $R$, each $g_{n} \in \mathscr{L}_{n}(\widetilde{T})(R)$ and each $f \in \widetilde{M}$, let $g_{n}^{*}(f) \in$ $\left(R[t] /\left(t^{n+1}\right)\right)^{\times}$denote the image of $f$ under the pullback map $k[\widetilde{M}] \rightarrow R[t] /\left(t^{n+1}\right)$ corresponding to the jet $g_{n}: \operatorname{Spec}\left(R[t] /\left(t^{n+1}\right)\right) \rightarrow \widetilde{T}$. We also use the analogous notation when $T$ and $M$ are in place of $\widetilde{T}$ and $\widetilde{M}$.

For each $w \in \sigma \cap N$ and $n \in \mathbb{N}$, let $G_{n}^{w}$ be the subgroup object of $\mathscr{L}_{n}(\widetilde{T})$ given by

$$
G_{n}^{w}(R)=\left\{g_{n} \in \mathscr{L}_{n}(\widetilde{T})(R) \mid g_{n}^{*}(p) t^{\langle w, p\rangle}=t^{\langle w, p\rangle} \in R[t] /\left(t^{n+1}\right) \text { for all } p \in P\right\} .
$$

For each $\widetilde{w} \in \widetilde{\sigma} \cap \widetilde{N}$ and $n \in \mathbb{N}$, let $H_{n}^{\widetilde{w}}$ be the subgroup object of $\mathscr{L}_{n}(\widetilde{T})$ given by

$$
H_{n}^{\widetilde{w}}(R)=\left\{g_{n} \in \mathscr{L}_{n}(\widetilde{T})(R) \mid g_{n}^{*}(f) t^{\langle\widetilde{w}, f\rangle}=t^{\langle\widetilde{w}, f\rangle} \in R[t] /\left(t^{n+1}\right) \text { for all } f \in F\right\} .
$$

By definition, $H_{n}^{\widetilde{w}}$ is a subgroup object of $G_{n}^{\beta(\widetilde{w})}$. We now show that these group objects are in fact algebraic groups.

Proposition 5.3. The subgroup objects $G_{n}^{w}$ and $H_{n}^{\widetilde{w}}$ are represented by closed subgroups of $\mathscr{L}_{n}(\widetilde{T})$. 
Proof. For each $f \in F$, let $\mathscr{L}_{n}(f): \mathscr{L}_{n}(\widetilde{T}) \rightarrow \mathscr{L}_{n}\left(\mathbb{G}_{m}\right)$ be the map induced by the character $\widetilde{T} \rightarrow \mathbb{G}_{m}$ corresponding to $f$. Then

$$
G_{n}^{w}=\bigcap_{p \in P}\left(\theta_{n-\langle w, p\rangle}^{n} \circ \mathscr{L}_{n}(p)\right)^{-1}
$$

and

$$
H_{n}^{\widetilde{w}}=\bigcap_{f \in F}\left(\theta_{n-\langle\widetilde{w}, f\rangle}^{n} \circ \mathscr{L}_{n}(f)\right)^{-1}
$$

where each $\theta_{n-n^{\prime}}^{n}: \mathscr{L}_{n}\left(\mathbb{G}_{m}\right) \rightarrow \mathscr{L}_{n-n^{\prime}}\left(\mathbb{G}_{m}\right)$ is the truncation morphism, each $1 \in \mathscr{L}_{n-n^{\prime}}\left(\mathbb{G}_{m}\right)$ is the identity element and, by convention, if $n-n^{\prime}<0$, we set $\mathscr{L}_{n-n^{\prime}}\left(\mathbb{G}_{m}\right)$ to be the single point group $\{1\}$.

Remark 5.4. Note that

$$
\mathscr{L}_{n}(G)(R)=\left\{g_{n} \in \mathscr{L}_{n}(\widetilde{T})(R) \mid g_{n}^{*}(p)=1 \in R[t] /\left(t^{n+1}\right) \text { for all } p \in P\right\},
$$

so for any $n \in \mathbb{N}$ and $w \in \sigma \cap N$, we have that $\mathscr{L}_{n}(G)$ is a closed subgroup of $G_{n}^{w}$.

We also prove the following characterisation of the $H_{n}^{\widetilde{w}}$ :

Proposition 5.5. Set $\widetilde{w} \in \widetilde{\sigma} \cap \widetilde{N}$ and $n \in \mathbb{N}$, and let $f_{1}, \ldots, f_{r}$ be the minimal generators of $F$. If $n \geq \max _{i=1}^{r}\left(2\left\langle\widetilde{w}, f_{i}\right\rangle-1\right)$, then

$$
H_{n}^{\widetilde{w}} \cong \mathbb{G}_{a}^{\sum_{i=1}^{r}\left\langle\widetilde{w}, f_{i}\right\rangle}
$$

as algebraic groups.

Proof. Let $R$ be a $k$-algebra. Then

$$
\begin{aligned}
H_{n}^{\widetilde{w}}(R) & =\left\{g_{n} \in \mathscr{L}_{n}(\widetilde{T})(R) \mid g_{n}^{*}(f) t^{\langle\widetilde{w}, f\rangle}=t^{\langle\widetilde{w}, f\rangle} \in R[t] /\left(t^{n+1}\right) \text { for all } f \in F\right\} \\
& =\left\{g_{n} \in \mathscr{L}_{n}(\widetilde{T})(R) \mid g_{n}^{*}\left(f_{i}\right) t^{\left\langle\widetilde{w}, f_{i}\right\rangle}=t^{\left\langle\widetilde{w}, f_{i}\right\rangle} \in R[t] /\left(t^{n+1}\right) \text { for all } i=1, \ldots, r\right\} \\
& \cong\left\{\left(g^{(i)}\right)_{i} \in\left(\left(R[t]\left(t^{n+1}\right)\right)^{\times}\right)^{r} \mid g^{(i)} t^{\left\langle\widetilde{w}, f_{i}\right\rangle}=t^{\left\langle\widetilde{w}, f_{i}\right\rangle} \in R[t] /\left(t^{n+1}\right) \text { for all } i\right\} .
\end{aligned}
$$

Since $n \geq 0$, the hypotheses guarantee that $n \geq\left\langle\widetilde{w}, f_{i}\right\rangle$ for all $i \in\{1, \ldots, r\}$. Thus if $i \in\{1, \ldots, r\}$ and $g^{(i)} \in\left(R[t] /\left(t^{n+1}\right)\right)^{\times}$, then

$$
g^{(i)} t^{\left\langle\widetilde{w}, f_{i}\right\rangle}=t^{\left\langle\widetilde{w}, f_{i}\right\rangle} \in R[t] /\left(t^{n+1}\right)
$$

if and only if

$$
g^{(i)}=1+a_{n-\left\langle\widetilde{w}, f_{i}\right\rangle+1} t^{n-\left\langle\widetilde{w}, f_{i}\right\rangle+1}+\cdots+a_{n} t^{n} \in R[t] /\left(t^{n+1}\right)
$$

for some $a_{n-\left\langle\widetilde{w}, f_{i}\right\rangle+1}, \ldots, a_{n} \in R$. The proposition thus follows from the fact that if $\left.a_{n-\langle\widetilde{w}}, f_{i}\right\rangle+1, \ldots, a_{n}$, $\left.b_{n-\langle\widetilde{w}}, f_{i}\right\rangle+1, \ldots, b_{n} \in R$, then in $R[t] /\left(t^{n+1}\right)$,

$$
\begin{aligned}
\left(1+a_{n-\left\langle\widetilde{w}, f_{i}\right\rangle+1} t^{n-\left\langle\widetilde{w}, f_{i}\right\rangle+1}+\cdots\right. & \left.+a_{n} t^{n}\right)\left(1+b_{n-\left\langle\widetilde{w}, f_{i}\right\rangle+1} t^{n-\left\langle\widetilde{w}, f_{i}\right\rangle+1}+\cdots+b_{n} t^{n}\right) \\
& =1+\left(a_{n-\left\langle\widetilde{w}, f_{i}\right\rangle+1}+b_{n-\left\langle\widetilde{w}, f_{i}\right\rangle+1}\right) t^{n-\left\langle\widetilde{w}, f_{i}\right\rangle+1}+\cdots+\left(a_{n}+b_{n}\right) t^{n},
\end{aligned}
$$


because for any $m_{1}, m_{2} \geq n-\left\langle\widetilde{w}, f_{i}\right\rangle+1$, we have

$$
m_{1}+m_{2} \geq 2 n-2\left\langle\widetilde{w}, f_{i}\right\rangle+2=2 n+1-\left(2\left\langle\widetilde{w}, f_{i}\right\rangle-1\right) \geq n+1 .
$$

We finish this subsection with the next two propositions, which characterise the group quotients $G_{n}^{w} / \mathscr{L}_{n}(G)$.

Proposition 5.6. Set $w \in \sigma \cap N$ and $n \in \mathbb{N}$. Then the group quotient $G_{n}^{w} / \mathscr{L}_{n}(G)$ has functor of points given by

$$
R \mapsto\left\{h_{n} \in \mathscr{L}_{n}(T)(R) \mid h_{n}^{*}(p) t^{\langle w, p\rangle}=t^{\langle w, p\rangle} \in R[t] /\left(t^{n+1}\right) \text { for all } p \in P\right\} .
$$

Proof. First, the subgroup object is represented by a closed subgroup of $\mathscr{L}_{n}(T)$ by an identical argument as in Proposition 5.3. Call this closed subgroup $H \subset \mathscr{L}_{n}(T)$. We will show that $G_{n}^{w} / \mathscr{L}_{n}(G) \cong H$ as schemes.

By the definition of $H$ and $G_{n}^{w}$, the closed subgroup $G_{n}^{w} \subset \mathscr{L}_{n}(\widetilde{T})$ is equal to the preimage of $H$ under the group homomorphism $\mathscr{L}_{n}(\widetilde{T}) \rightarrow \mathscr{L}_{n}(T)$ obtained by applying the functor $\mathscr{L}_{n}$ to the group homomorphism $\left.\widetilde{\pi}\right|_{\widetilde{T}}: \widetilde{T} \rightarrow T$. Thus we obtain a group homomorphism $G_{n}^{w} \rightarrow H$. Endow $H$ with the $G_{n}^{w}$-action obtained by left multiplication after $G_{n}^{w} \rightarrow H$.

For any $k$-algebra $R$ and $g_{n} \in G_{n}^{w}(R)$, we have that $g_{n} \in \mathscr{L}_{n}(G)(R)$ if and only if $g_{n}^{*}(p)=1$ for all $p \in P$, which is equivalent to $g_{n}$ being in the kernel of $G_{n}^{w} \rightarrow H$. Therefore the scheme-theoretic stabiliser of the identity $1 \in H(k)$ is equal to $\mathscr{L}_{n}(G)$. Thus by [DG70, Chapter III, Section 3, Proposition 5.2], we have a locally closed embedding

$$
G_{n}^{w} / \mathscr{L}_{n}(G) \hookrightarrow H
$$

whose image, as a set, is equal to the image of the map of underlying sets $G_{n}^{w} \rightarrow H$. Since $k$ has characteristic 0 so that $H$ is reduced, we only need to show that $G_{n}^{w} \rightarrow H$ is surjective on underlying sets, which will follow if we can show that $\mathscr{L}_{n}(\widetilde{T}) \rightarrow \mathscr{L}_{n}(T)$ is surjective on underlying sets. The latter follows immediately from Corollary 3.22, which implies that $\mathscr{L}_{n}(\widetilde{T}) \rightarrow \mathscr{L}_{n}(T)$ is a $\mathscr{L}_{n}(G)$-torsor.

Proposition 5.7. Set $w \in \sigma \cap N$. Then there exist $n_{w}^{\prime}, j_{w}^{\prime} \in \mathbb{N}$ such that for all $n \geq n_{w}^{\prime}$, we have an isomorphism of schemes

$$
G_{n}^{w} / \mathscr{L}_{n}(G) \cong \mathbb{A}_{k}^{j_{w}^{\prime}}
$$

Proof. Let $p_{1}, \ldots, p_{s}$ be a set of generators of the semigroup $P$ and set

$$
n_{w}^{\prime}=\max _{1 \leq i \leq s}\left(2\left\langle w, p_{i}\right\rangle-1\right)
$$

and $m=\max _{1 \leq i \leq s}\left\langle w, p_{i}\right\rangle$. Consider the affine space $\mathbb{A}_{k}^{m s}$ with coordinates $\left(x_{\ell}^{(i)}\right)_{i \in\{1, \ldots, s\}, \ell \in\{1, \ldots, m\}}$. Let $V$ be the linear subspace of $\mathbb{A}_{k}^{m s}$ defined by the vanishing of all $x_{\ell}^{(i)}$ for $\ell>\left\langle w, p_{i}\right\rangle$ and the vanishing of all $\sum_{i=1}^{s} m_{i} x_{\ell}^{(i)}$ for all $\ell \in\{1, \ldots, m\}$ and all $m_{1}, \ldots, m_{s} \in \mathbb{Z}$ such that

$$
\sum_{i=1}^{s} m_{i} p_{i}=0 \in P^{\mathrm{gp}}=M .
$$

Set $j_{w}^{\prime}=\operatorname{dim} V$. It suffices to show that for all $n \geq n_{w}^{\prime}$, we have that $G_{n}^{w} / \mathscr{L}_{n}(G) \cong V$ as schemes.

Let $n \geq n_{w}^{\prime}$ and let $H$ be the closed subgroup of $\mathscr{L}_{n}(T)$ representing the subgroup object in the statement of Proposition 5.6. By Proposition 5.6, it is sufficient to prove that $H \cong V$ as schemes. 
Let $H \rightarrow \mathbb{A}_{k}^{m s}$ be the morphism that, for each $k$-algebra $R$, takes $h_{n} \in H(R)$ to $\left(a_{n-\ell+1}^{(i)}\right)_{i, \ell} \in R^{m s}=$ $\mathbb{A}_{k}^{m s}(R)$, where for all $i, \ell$ we have that $a_{n-\ell+1}^{(i)}$ is the coefficient of $t^{n-\ell+1}$ in $p_{i}\left(h_{n}\right) \in R[t] /\left(t^{n+1}\right)$. This morphism $H \rightarrow \mathbb{A}_{k}^{m s}$ factors through an isomorphism $H \stackrel{\sim}{\rightarrow} V$ by the definition of $H$, the construction of $V$ and the fact that $n \geq n_{w}^{\prime}$ implies that for any $k$-algebra $R$, any $m_{1}, \ldots, m_{s} \in \mathbb{Z}$ and any $\left(a_{n-\ell+1}^{(i)}\right)_{i, \ell} \in R^{m s}$, we have that

$$
\begin{aligned}
\prod_{i=1}^{s}\left(1+a_{n-m+1}^{(i)} t^{n-m+1}\right. & \left.+\cdots+a_{n}^{(i)} t^{n}\right)^{m_{i}} \\
& =1+\left(\sum_{i=1}^{s} m_{i} a_{n-m+1}^{(i)}\right) t^{n-m+1}+\cdots+\left(\sum_{i=1}^{s} m_{i} a_{n}^{(i)}\right) t^{n} \in R[t] /\left(t^{n+1}\right) .
\end{aligned}
$$

\subsection{Components of the fibres}

In this subsection, we will control fibres of each $\mathscr{L}_{n}(\pi): \mathcal{X} \rightarrow X$ by controlling the connected components of the fibres of each map $\mathscr{L}_{n}(\widetilde{\pi}): \mathscr{L}_{n}(\widetilde{X}) \rightarrow \mathscr{L}_{n}(X)$. In particular, we will show that for $n$ sufficiently large and $\varphi \in \mathscr{L}(X)$ with $\operatorname{trop}(\varphi)=w \in \sigma \cap N$, the connected components of $\mathscr{L}_{n}(\widetilde{\pi})^{-1}\left(\theta_{n}(\varphi)\right)$ are indexed by $\beta^{-1}(w)$. To do this, we will define analogues of the map trop for the jet schemes $\mathscr{L}_{n}(\widetilde{X})$.

For any $n \in \mathbb{N}$, let $\mathbb{N}_{n}=\{0,1, \ldots, n, \infty\}$ with the monoid structure making

$$
\mathbb{N} \cup\{\infty\} \rightarrow \mathbb{N}_{n}: \ell \mapsto \begin{cases}\ell, & \ell \leq n, \\ \infty, & \ell>n\end{cases}
$$

a map of monoids. For any $\widetilde{w} \in \operatorname{Hom}(F, \mathbb{N} \cup\{\infty\})$, let $\widetilde{w}_{n} \in \operatorname{Hom}\left(F, \mathbb{N}_{n}\right)$ be the composition of $\widetilde{w}: F \rightarrow \mathbb{N} \cup\{\infty\}$ with $\mathbb{N} \cup\{\infty\} \rightarrow \mathbb{N}_{n}$.

Remark 5.8. In what follows, for each $k$-algebra $R$, each $\widetilde{\psi}_{n} \in \mathscr{L}_{n}(\widetilde{X})(R)$ and each $f \in F$, let $\widetilde{\psi}_{n}^{*}(f) \in R[t] /\left(t^{n+1}\right)$ denote the image of $f$ under the pullback map $k[F] \rightarrow R[t] /\left(t^{n+1}\right)$ corresponding to the jet $\widetilde{\psi}_{n}: \operatorname{Spec}\left(R[t] /\left(t^{n+1}\right)\right) \rightarrow \widetilde{X}$. We use the analogous notation when $X$ and $P$ are in place of $\widetilde{X}$ and $F$.

For any field extension $k^{\prime}$ of $k$ and $\widetilde{\psi}_{n} \in \mathscr{L}_{n}(\widetilde{X})\left(k^{\prime}\right)$, define

$$
\operatorname{trop}_{n}\left(\widetilde{\psi}_{n}\right) \in \operatorname{Hom}\left(F, \mathbb{N}_{n}\right)
$$

to be the map taking each $f \in F$ to the $t$-order of vanishing of $\widetilde{\psi}_{n}^{*}(f) \in k^{\prime}[t] /\left(t^{n+1}\right)$. Also define the map

$$
\operatorname{trop}_{n}: \mathscr{L}_{n}(\widetilde{X}) \rightarrow \operatorname{Hom}\left(F, \mathbb{N}_{n}\right)
$$

by considering each $\widetilde{\psi}_{n} \in \mathscr{L}(\widetilde{X})$ as a point valued in its residue field.

Remark 5.9. As a direct consequence of the definition, trop $_{n}$ is compatible with field extensions. In other words, for any field extension $k^{\prime}$ of $k$ and $\widetilde{\psi}_{n} \in \mathscr{L}_{n}(\widetilde{X})\left(k^{\prime}\right)$, we have that trop $n\left(\widetilde{\psi}_{n}\right)$ is equal to $\operatorname{trop}_{n}$ applied to the image of $\widetilde{\psi}_{n}$ in the underlying set of $\mathscr{L}_{n}(\widetilde{X})$. 
Remark 5.10. For any $\widetilde{\psi} \in \mathscr{L}(\widetilde{X})\left(k^{\prime}\right)$,

$$
(\operatorname{trop}(\widetilde{\psi}))_{n}=\operatorname{trop}_{n}\left(\theta_{n}(\widetilde{\psi})\right) .
$$

Note that because $\widetilde{X}$ is an affine space and thus is smooth, all jets of $\widetilde{X}$ are truncations of arcs of $\widetilde{X}$, so this equality in fact determines trop ${ }_{n}$.

We next stratify the fibre of $\mathscr{L}_{n}(\tilde{\pi})$ according to the value of trop $n$. We show that for $n$ sufficiently large, each stratum is a union of connected components.

Lemma 5.11. For each $f \in F$, the map

$$
\mathscr{L}_{n}(\widetilde{X}) \rightarrow \mathbb{N}_{n}: \widetilde{\psi}_{n} \mapsto\left(\operatorname{trop}_{n}\left(\widetilde{\psi}_{n}\right)\right)(f)
$$

is upper semicontinuous.

Proof. Since $\widetilde{X}$ is affine, $\mathscr{L}_{n}(\widetilde{X})=\operatorname{Spec}(R)$ for some $k$-algebra $R$. Let $\Psi$ be the universal family of $\mathscr{L}_{n}(\widetilde{X})$ - that is, let

$$
\Psi: \operatorname{Spec}\left(R[t] /\left(t^{n+1}\right)\right) \rightarrow \widetilde{X}
$$

be the $R$-valued jet corresponding to the identity $\operatorname{Spec}(R)=\mathscr{L}_{n}(\widetilde{X})$, and let $\Psi^{*}(f) \in R[t] /\left(t^{n+1}\right)$ be the result of pulling back $f$ along $\Psi$. Write

$$
\Psi^{*}(f)=a_{0}+\cdots+a_{n} t^{n}
$$

where $a_{0}, \ldots, a_{n} \in R$. Then the jets $\widetilde{\psi}_{n} \in \mathscr{L}_{n}(\widetilde{X})$ with $\left(\operatorname{trop}_{n}\left(\widetilde{\psi}_{n}\right)\right)(f) \geq \ell$ are exactly the points of $\operatorname{Spec}(R)$ where $a_{0}, \ldots, a_{\ell-1}$ vanish.

Proposition 5.12. Set $w \in \sigma \cap N$. Then there exists some $n_{0} \in \mathbb{N}$ such that for any $n \geq n_{0}$, any field extension $k^{\prime}$ of $k$ and any $\varphi \in \mathscr{L}(X)\left(k^{\prime}\right)$ with $\operatorname{trop}(\varphi)=w$, we have that the restriction of $\operatorname{trop}_{n}: \mathscr{L}_{n}(\widetilde{X}) \rightarrow \operatorname{Hom}\left(F, \mathbb{N}_{n}\right)$ to the fibre $\mathscr{L}_{n}(\widetilde{\pi})^{-1}\left(\theta_{n}(\varphi)\right)$ is locally constant.

Proof. Set $f \in F$. We will show that there exists some $n_{f} \in \mathbb{N}$ such that for any $n \geq n_{f}$, any field extension $k^{\prime}$ of $k$ and any $\varphi \in \mathscr{L}(X)\left(k^{\prime}\right)$ with $\operatorname{trop}(\varphi)=w$, we have that the restriction of

$$
\mathscr{L}_{n}(\widetilde{X}) \rightarrow \mathbb{N}_{n}: \widetilde{\psi}_{n} \mapsto\left(\operatorname{trop}_{n}\left(\widetilde{\psi}_{n}\right)\right)(f)
$$

to the fibre $\mathscr{L}_{n}(\widetilde{\pi})^{-1}\left(\theta_{n}(\varphi)\right)$ is locally constant.

By Proposition 2.14, there exists some $f^{\prime} \in F$ such that $f+f^{\prime} \in P$. Set

$$
n_{f}=\left\langle w, f+f^{\prime}\right\rangle \text {. }
$$

Let $n \geq n_{f}$, let $k^{\prime}$ be a field extension of $k$ and let $\varphi \in \mathscr{L}(X)\left(k^{\prime}\right)$ with $\operatorname{trop}(\varphi)=w$. Since $\mathscr{L}_{n}(\tilde{\pi})$ : $\mathscr{L}_{n}(\widetilde{X}) \rightarrow \mathscr{L}_{n}(X)$ is finite type, it is sufficient to show that on any irreducible component $C$ of the fibre $\mathscr{L}_{n}(\tilde{\pi})^{-1}\left(\theta_{n}(\varphi)\right)$, the map

$$
\alpha: C \rightarrow \mathbb{N}_{n}: \widetilde{\psi}_{n} \mapsto\left(\operatorname{trop}_{n}\left(\widetilde{\psi}_{n}\right)\right)(f)
$$


is constant. By Lemma 5.11, $\alpha$ and the map

$$
\gamma: C \rightarrow \mathbb{N}_{n}: \widetilde{\psi}_{n} \mapsto\left(\operatorname{trop}_{n}\left(\widetilde{\psi}_{n}\right)\right)\left(f^{\prime}\right)
$$

are upper semicontinuous. Also, for all $\widetilde{\psi}_{n} \in C$,

$$
\alpha\left(\widetilde{\psi}_{n}\right)+\gamma\left(\widetilde{\psi}_{n}\right)=\left(\operatorname{trop}_{n}\left(\widetilde{\psi}_{n}\right)\right)\left(f+f^{\prime}\right)=\left\langle w, f+f^{\prime}\right\rangle_{n},
$$

where $\left\langle w, f+f^{\prime}\right\rangle_{n}$ is the image of $\left\langle w, f+f^{\prime}\right\rangle$ in $\mathbb{N}_{n}$. Thus the sum of $\alpha$ and $\gamma$ is a constant function. Furthermore, because $n \geq\left\langle w, f+f^{\prime}\right\rangle$, the sum of $\alpha$ and $\gamma$ is not equal to $\infty$. Therefore, $\alpha$ is constant by upper semicontinuity of $\alpha$ and $\gamma$ and the fact that $C$ is irreducible.

Now the proposition is obtained by taking $n_{0}$ to be larger than all $n_{f}$ as $f$ vary over the minimal generators of $F$.

For any $n \in \mathbb{N}$, any $\widetilde{w} \in \widetilde{\sigma} \cap \widetilde{N}$, any field extension $k^{\prime}$ of $k$ and any $\varphi \in \mathscr{L}(X)\left(k^{\prime}\right)$, let $C_{n}^{\widetilde{w}}(\varphi)$ denote the locus in $\mathscr{L}_{n}(\widetilde{\pi})^{-1}\left(\theta_{n}(\varphi)\right)$ where trop ${ }_{n}$ is equal to $\widetilde{w}_{n}$. We will be interested in the case where $C_{n}^{\widetilde{w}}(\varphi)$ is a union of connected components of $\mathscr{L}_{n}(\widetilde{\pi})^{-1}\left(\theta_{n}(\varphi)\right)$. In that case, we will give $C_{n}^{\widetilde{w}}(\varphi)$ its reduced scheme structure.

Proposition 5.13. Set $w \in \sigma \cap N$. Then there exists some $n_{1} \in \mathbb{N}$ such that for any $n \geq n_{1}$, any field extension $k^{\prime}$ of $k$, any $\varphi \in \mathscr{L}(X)\left(k^{\prime}\right)$ with $\operatorname{trop}(\varphi)=w$ and any $\widetilde{w} \in \beta^{-1}(w)$,

1. $C_{n}^{\widetilde{w}}(\varphi)$ is a union of connected components of $\mathscr{L}_{n}(\widetilde{\pi})^{-1}\left(\theta_{n}(\varphi)\right)$, and we have an isomorphism of schemes

$$
\mathscr{L}_{n}(\widetilde{\pi})^{-1}\left(\theta_{n}(\varphi)\right)_{\mathrm{red}} \cong \bigsqcup_{\widetilde{w}^{\prime} \in \beta^{-1}(w)} C_{n}^{\widetilde{w}^{\prime}}(\varphi)
$$

2. $C_{n}^{\widetilde{w}}(\varphi)$ is invariant under the action of $G_{n}^{w} \otimes_{k} k^{\prime}$ on $\mathscr{L}_{n}(\widetilde{X}) \otimes_{k} k^{\prime}$;

3. for each field extension $k^{\prime \prime}$ of $k^{\prime}$, the action of $\left(G_{n}^{w} \otimes_{k} k^{\prime}\right)\left(k^{\prime \prime}\right)$ on $C_{n}^{\widetilde{w}}(\varphi)\left(k^{\prime \prime}\right)$ is transitive; and

4. for each field extension $k^{\prime \prime}$ of $k^{\prime}$, the scheme-theoretic stabiliser of any $k^{\prime \prime}$-point of $C_{n}^{\widetilde{w}}(\varphi)$ under the $G_{n}^{w} \otimes_{k} k^{\prime}$-action is equal to $H_{n}^{\widetilde{w}} \otimes_{k} k^{\prime \prime}$.

Remark 5.14. In the statement of Proposition 5.13, the action of $G_{n}^{w}$ on $\mathscr{L}_{n}(\widetilde{X})$ is the one induced by the inclusion $G_{n}^{w} \hookrightarrow \widetilde{T}$ and the functor $\mathscr{L}_{n}$ applied to the toric action $\widetilde{T} \times_{k} \widetilde{X} \rightarrow \widetilde{X}$.

Proof. Let $f_{1}, \ldots, f_{r}$ be the minimal generators of $F$, and let $p_{1}, \ldots, p_{s}$ be a set of generators for the semigroup $P$. Let $n_{0} \in \mathbb{N}$ be as in the statement of Proposition 5.12, and let $n_{1} \geq n_{0}$ be such that $n_{1} \geq\left\langle w, p_{i}\right\rangle$ for all $i \in\{1, \ldots, s\}$ and $n_{1} \geq\left\langle\widetilde{w}, f_{i}\right\rangle$ for all $i \in\{1, \ldots, r\}$ and $\widetilde{w} \in \beta^{-1}(w)$. Note that we can choose such an $n_{1}$ by Proposition 2.15 .

Let $n \geq n_{1}$, let $k^{\prime}$ be a field extension of $k$ and set $\varphi \in \mathscr{L}(X)\left(k^{\prime}\right)$ with $\operatorname{trop}(\varphi)=w$. We begin by proving the first part of the proposition.

1. By our choice of $n_{0}$ and Proposition 5.12, $C_{n}^{\widetilde{w}}(\varphi)$ is a union of connected components of $\mathscr{L}_{n}(\widetilde{\pi})^{-1}\left(\theta_{n}(\varphi)\right)$, so it suffices to prove that $\mathscr{L}_{n}(\tilde{\pi})^{-1}\left(\theta_{n}(\varphi)\right)$ and $\bigsqcup_{\widetilde{w}^{\prime} \in \beta^{-1}(w)} C_{n}^{\widetilde{w}^{\prime}}(\varphi)$ are equal as sets.

Set $\widetilde{w} \in \widetilde{\sigma} \cap \widetilde{N}$. We first show that if $C_{n}^{\widetilde{w}} \neq \emptyset$, then $\widetilde{w} \in \beta^{-1}(w)$. If $\widetilde{\psi}_{n} \in \mathscr{L}_{n}(\widetilde{\pi})^{-1}\left(\theta_{n}(\varphi)\right)$, then for all $i \in\{1, \ldots, s\}$,

$$
\left(\operatorname{trop}_{n}\left(\widetilde{\psi}_{n}\right)\right)\left(p_{i}\right)=\left\langle w, p_{i}\right\rangle_{n}
$$

where $\left\langle w, p_{i}\right\rangle_{n}$ is the image of $\left\langle w, p_{i}\right\rangle$ in $\mathbb{N}_{n}$. Since $n \geq n_{1} \geq\left\langle w, p_{i}\right\rangle$, this implies that if $\widetilde{\psi}_{n} \in C_{n}^{\widetilde{w}}$, then $\left\langle\widetilde{w}, p_{i}\right\rangle=\left\langle w, p_{i}\right\rangle$ for all $i \in\{1, \ldots, s\}$, and so $\beta(\widetilde{w})=w$. 
Having shown $C_{n}^{\widetilde{w}}(\varphi)=\emptyset$ whenever $\widetilde{w} \notin \beta^{-1}(w)$, we need only show that if $\widetilde{w}_{1}, \widetilde{w}_{2}$ are distinct elements of $\beta^{-1}(w)$; then $\left(\widetilde{w}_{1}\right)_{n} \neq\left(\widetilde{w}_{2}\right)_{n}$. This follows from the fact that for each $i \in\{1, \ldots, r\}$ and $\widetilde{w} \in \beta^{-1}(w)$,

$$
\left\langle\widetilde{w}, f_{i}\right\rangle \leq n_{1} \leq n
$$

For the rest of this proof, set $\widetilde{w} \in \beta^{-1}(w)$ and set the following notation: for each field extension $k^{\prime \prime}$ of $k^{\prime}$, let $\varphi_{n, k^{\prime \prime}} \in \mathscr{L}_{n}(X)\left(k^{\prime \prime}\right)$ be the composition

$$
\operatorname{Spec}\left(k^{\prime \prime}[t] /\left(t^{n+1}\right)\right) \rightarrow \operatorname{Spec}\left(k^{\prime}[t] /\left(t^{n+1}\right)\right) \stackrel{\theta_{n}(\varphi)}{\longrightarrow} X,
$$

where the map $\operatorname{Spec}\left(k^{\prime \prime}[t] / t^{n+1}\right) \rightarrow \operatorname{Spec}\left(k^{\prime}[t] / t^{n+1}\right)$ is given by the $k^{\prime}$-algebra map $k^{\prime}[t] /\left(t^{n+1}\right) \rightarrow$ $k^{\prime \prime}[t] /\left(t^{n+1}\right): t \mapsto t$. Note that the $k^{\prime \prime}$-points of $C_{n}^{\widetilde{w}}(\varphi)$ are precisely those $\widetilde{\psi}_{n}: \operatorname{Spec}\left(k^{\prime \prime}[t] /\left(t^{n+1}\right)\right) \rightarrow$ $\widetilde{X}$ such that $\operatorname{trop}_{n}\left(\widetilde{\psi}_{n}\right)=\widetilde{w}_{n}$ and the composition $\operatorname{Spec}\left(k^{\prime \prime}[t] /\left(t^{n+1}\right)\right) \stackrel{\widetilde{\psi}_{n}}{\longrightarrow} \widetilde{X} \rightarrow X$ is equal to $\varphi_{n, k^{\prime \prime}}$. We now prove the remaining parts of the proposition.

2. Since $C_{n}^{\widetilde{w}}(\varphi)$ is reduced by definition, it suffices to show that for each field extension $k^{\prime \prime}$ of $k^{\prime}$, we have that $C_{n}^{\widetilde{w}}(\varphi)\left(k^{\prime \prime}\right)$ is invariant under the action of $G_{n}^{w}\left(k^{\prime \prime}\right)$ on $\mathscr{L}_{n}(\widetilde{X})\left(k^{\prime \prime}\right)$.

Let $k^{\prime \prime}$ be a field extension of $k^{\prime}$, set $\widetilde{\psi}_{n} \in C_{n}^{\widetilde{w}}(\varphi)\left(k^{\prime \prime}\right)$ and set $g_{n} \in G_{n}^{w}\left(k^{\prime \prime}\right)$. Then for all $f \in F$,

$$
\left(g_{n} \cdot \widetilde{\psi}_{n}\right)^{*}(f)=g_{n}^{*}(f) \widetilde{\psi}_{n}^{*}(f)
$$

has the same $t$-order of vanishing as $\widetilde{\psi}_{n}^{*}(f)$, because $g_{n}^{*}(f) \in k^{\prime \prime}[t] /\left(t^{n+1}\right)$ is a unit. Thus

$$
\operatorname{trop}_{n}\left(g_{n} \cdot \widetilde{\psi}_{n}\right)=\operatorname{trop}_{n}\left(\widetilde{\psi}_{n}\right)=\widetilde{w}_{n}
$$

We also have that for all $p \in P$,

$$
\left(g_{n} \cdot \widetilde{\psi}_{n}\right)^{*}(p)=g_{n}^{*}(p) \widetilde{\psi}_{n}^{*}(p)=g_{n}^{*}(p) \varphi_{n, k^{\prime \prime}}^{*}(p)=\varphi_{n, k^{\prime \prime}}^{*}(p),
$$

where the last equality follows from the definition of $G_{n}^{w}$ and the fact that $\operatorname{trop}(\varphi)=w$ implies that $\varphi_{n, k^{\prime \prime}}^{*}(p)$ is divisible by $t^{\langle w, p\rangle}$. Therefore the composition Spec $\left(k^{\prime \prime}[t] /\left(t^{n+1}\right)\right) \stackrel{g_{n} \cdot \widetilde{\psi}_{n}}{\longrightarrow} \widetilde{X} \rightarrow X$ is equal to $\varphi_{n, k^{\prime \prime}}$. Thus

$$
g_{n} \cdot \widetilde{\psi}_{n} \in C_{n}^{\widetilde{w}}(\varphi)\left(k^{\prime \prime}\right)
$$

3. Let $k^{\prime \prime}$ be a field extension of $k^{\prime}$, and set $\widetilde{\psi}_{n}, \widetilde{\psi}_{n}^{\prime} \in C_{n}^{\widetilde{w}}(\varphi)\left(k^{\prime \prime}\right)$. We will first show that there exists some $g_{n} \in \mathscr{L}_{n}(\widetilde{T})\left(k^{\prime \prime}\right)$ such that $g_{n} \cdot \widetilde{\psi}_{n}=\widetilde{\psi}_{n}^{\prime}$.

Let $f_{1}, \ldots, f_{r}$ be the minimal generators of $F$. For each $i \in\{1, \ldots, r\}$, we have that $\widetilde{\psi}_{n}^{*}\left(f_{i}\right)$ and $\left(\widetilde{\psi}_{n}^{\prime}\right)^{*}\left(f_{i}\right)$ have the same $t$-order of vanishing because $\operatorname{trop}_{n}\left(\widetilde{\psi}_{n}\right)=\widetilde{w}=\operatorname{trop}_{n}\left(\widetilde{\psi}_{n}^{\prime}\right)$. Thus there exists a unit $g^{(i)} \in k[t] /\left(t^{n+1}\right)$ such that

$$
g^{(i)} \widetilde{\psi}_{n}^{*}\left(f_{i}\right)=\left(\widetilde{\psi}_{n}^{\prime}\right)^{*}\left(f_{i}\right)
$$

Letting $g_{n} \in \mathscr{L}_{n}(\widetilde{T})\left(k^{\prime \prime}\right)$ be such that $g_{n}^{*}\left(f_{i}\right)=g^{(i)}$ for all $i \in\{1, \ldots, r\}$,

$$
g_{n} \cdot \widetilde{\psi}_{n}=\widetilde{\psi}_{n}^{\prime}
$$


Now it suffices to show that if $g_{n} \in \mathscr{L}_{n}(\widetilde{T})\left(k^{\prime \prime}\right)$ and $\widetilde{\psi}_{n}, g_{n} \cdot \widetilde{\psi}_{n} \in C_{n}^{\widetilde{w}}(\varphi)\left(k^{\prime \prime}\right)$, then $g_{n} \in G_{n}^{w}\left(k^{\prime \prime}\right)$. For all $p \in P$,

$$
g_{n}^{*}(p) \varphi_{n, k^{\prime \prime}}^{*}(p)=g_{n}^{*}(p) \widetilde{\psi}_{n}^{*}(p)=\left(g_{n} \cdot \widetilde{\psi}_{n}\right)^{*}(p)=\varphi_{n, k^{\prime \prime}}^{*}(p),
$$

so because $\varphi_{n, k^{\prime \prime}}^{*}(p)$ is a unit multiple of $t^{\langle w, p\rangle}$, we have that $g_{n} \in G_{n}^{w}\left(k^{\prime \prime}\right)$ by definition.

4. Let $k^{\prime \prime}$ be a field extension of $k^{\prime}$, set $\widetilde{\psi}_{n} \in C_{n}^{\widetilde{w}}(\varphi)\left(k^{\prime \prime}\right)$, and for any $k^{\prime \prime}$-algebra $R$, let $\widetilde{\psi}_{n, R} \in$ $C_{n}^{\widetilde{w}}(\varphi)(R)$ be the composition

$$
\operatorname{Spec}\left(R[t] /\left(t^{n+1}\right)\right) \rightarrow \operatorname{Spec}\left(k^{\prime \prime}[t] /\left(t^{n+1}\right)\right) \stackrel{\widetilde{\psi}_{n}}{\longrightarrow} \widetilde{X} .
$$

Set $g_{n} \in G_{n}^{w}(R)$. Then for each $f \in F$, we have that $\widetilde{\psi}_{n, R}^{*}(f) \in R[t] /\left(t^{n+1}\right)$ is the product of a unit in $k^{\prime \prime}[t] /\left(t^{n+1}\right)$ and $t^{\langle\widetilde{w}, f\rangle}$, so

$$
g_{n}^{*}(f) \widetilde{\psi}_{n, R}^{*}(f)=\widetilde{\psi}_{n, R}^{*}(f) \Longleftrightarrow g_{n}^{*}(f) t^{\langle\widetilde{w}, f\rangle}=t^{\langle\widetilde{w}, f\rangle} .
$$

Therefore $g_{n}$ is in the stabiliser of $\widetilde{\psi}_{n}$ if and only if $g_{n} \in H_{n}^{\widetilde{w}}(R)$.

In the next proposition, we use Theorem 4.9 and Proposition 5.13 to control the reduced fibres of each $\mathscr{L}_{n}(\pi): \mathscr{L}_{n}(\mathcal{X}) \rightarrow \mathscr{L}_{n}(X)$.

Proposition 5.15. Set $w \in \sigma \cap N$. Then there exists some $n_{2} \in \mathbb{N}$ such that for any $n \geq n_{2}$, any field extension $k^{\prime}$ of $k$ and any $\varphi \in \mathscr{L}(X)\left(k^{\prime}\right)$ with $\operatorname{trop}(\varphi)=w$, we have

$$
\mathscr{L}_{n}(\pi)^{-1}\left(\theta_{n}(\varphi)\right)_{\mathrm{red}} \cong\left(\bigsqcup_{\widetilde{w} \in \beta^{-1}(w)}\left[\left(G_{n}^{w} / \mathscr{L}_{n}(G)\right) / H_{n}^{\widetilde{w}}\right]\right) \otimes_{k} k^{\prime},
$$

where $H_{n}^{\widetilde{w}}$ acts on $G_{n}^{w} / \mathscr{L}_{n}(G)$ via the group homomorphism $H_{n}^{\widetilde{w}} \hookrightarrow G_{n}^{w} \rightarrow G_{n}^{w} / \mathscr{L}_{n}(G)$ and left multiplication.

Proof. Let $f_{1}, \ldots, f_{r}$ be the minimal generators of $F$. Let $n_{1}$ be as in the statement of Proposition 5.13, and let $n_{2} \geq n_{1}$ be such that $n_{2} \geq \max _{i=1}^{r}\left(2\left\langle\widetilde{w}, f_{i}\right\rangle-1\right)$ for all $\widetilde{w} \in \beta^{-1}(w)$. Note that we can choose such an $n_{2}$ by Proposition 2.15.

Let $n \geq n_{2}$, let $k^{\prime}$ be a field extension of $k$ and let $\varphi \in \mathscr{L}(X)\left(k^{\prime}\right)$ be such that $\operatorname{trop}(\varphi)=w$. By Corollary 3.22, there exists an isomorphism $\mathscr{L}_{n}(\mathcal{X}) \stackrel{\sim}{\rightarrow}\left[\mathscr{L}_{n}(\widetilde{X}) / \mathscr{L}_{n}(G)\right]$ such that the following diagram commutes:

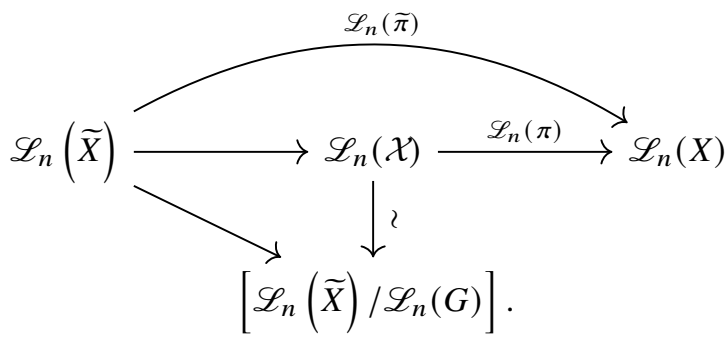

Therefore

$$
\mathscr{L}_{n}(\pi)^{-1}\left(\theta_{n}(\varphi)\right) \cong\left[\mathscr{L}_{n}(\widetilde{\pi})^{-1}\left(\theta_{n}(\varphi)\right) /\left(\mathscr{L}_{n}(G) \otimes_{k} k^{\prime}\right)\right]
$$


where $\mathscr{L}_{n}(G) \otimes_{k} k^{\prime}$ acts on $\mathscr{L}_{n}(\widetilde{\pi})^{-1}\left(\theta_{n}(\varphi)\right)$ by restriction of its action on $\mathscr{L}_{n}(\widetilde{X}) \otimes_{k} k^{\prime}$, which itself is the action induced by the inclusion $\mathscr{L}_{n}(G) \hookrightarrow \mathscr{L}_{n}(\widetilde{T})$ and the functor $\mathscr{L}_{n}$ applied to the toric action $\widetilde{T} \times_{k} \widetilde{X} \rightarrow \widetilde{X}$. Thus by Remark 5.4 and Remark 5.14, the action of $\mathscr{L}_{n}(G) \otimes_{k} k^{\prime}$ on $\mathscr{L}_{n}(\widetilde{\pi})^{-1}\left(\theta_{n}(\varphi)\right)$ is the restriction of the action on $\mathscr{L}_{n}(\widetilde{X}) \otimes_{k} k^{\prime}$ induced by the inclusion $\mathscr{L}_{n}(G) \otimes_{k} k^{\prime} \hookrightarrow G_{n}^{w} \otimes_{k} k^{\prime}$ and the action of $G_{n}^{w} \otimes_{k} k^{\prime}$ on $\mathscr{L}_{n}(\widetilde{X}) \otimes_{k} k^{\prime}$. Thus Proposition 5.13(2) implies that for all $\widetilde{w} \in \beta^{-1}(w)$, we have that $C_{n}^{\widetilde{w}}(\varphi)$ is invariant under the $\mathscr{L}_{n}(G) \otimes_{k} k^{\prime}$ action, so by Proposition 5.13(1),

$$
\mathscr{L}_{n}(\pi)^{-1}\left(\theta_{n}(\varphi)\right)_{\mathrm{red}} \cong \bigsqcup_{\widetilde{w} \in \beta^{-1}(w)}\left[C_{n}^{\widetilde{w}}(\varphi) /\left(\mathscr{L}_{n}(G) \otimes_{k} k^{\prime}\right)\right] .
$$

Set $\widetilde{w} \in \beta^{-1}(w)$. It will be sufficient to prove that

$$
\left[C_{n}^{\widetilde{w}}(\varphi) /\left(\mathscr{L}_{n}(G) \otimes_{k} k^{\prime}\right)\right] \cong\left[\left(G_{n}^{w} / \mathscr{L}_{n}(G)\right) / H_{n}^{\widetilde{w}}\right] \otimes_{k} k^{\prime}
$$

We begin by establishing that $C_{n}^{\widetilde{w}}(\varphi) /\left(\mathscr{L}_{n}\left(G^{\prime}\right) \otimes_{k} k^{\prime}\right)$ is an affine scheme with a $k^{\prime}$-point. Since $G$ is a diagonalisable group scheme, we have $G \cong T^{\prime} \times_{k} G^{\prime}$, where $T^{\prime}$ is a torus and $G^{\prime}$ is a finite group. This yields an identification

$$
\mathscr{L}_{n}(G) \otimes_{k} k^{\prime} \cong\left(\mathscr{L}_{n}\left(T^{\prime}\right) \times_{k} \mathscr{L}_{n}\left(G^{\prime}\right)\right) \otimes_{k} k^{\prime}
$$

Note that $\mathscr{L}_{n}\left(G^{\prime}\right) \cong G^{\prime}$, since $G^{\prime}$ is a finite group. By Proposition 5.5, our choice of $n_{2}$ and the fact that $k$ has characteristic 0 , we then see

$$
\{1\}=\mathscr{L}_{n}\left(G^{\prime}\right) \cap H_{n}^{\widetilde{w}} \subset G_{n}^{w} .
$$

Thus Proposition 5.13(4) implies that $\mathscr{L}_{n}\left(G^{\prime}\right) \otimes_{k} k^{\prime}$ acts freely on $C_{n}^{\widetilde{w}}(\varphi)$. Note that $C_{n}^{\widetilde{w}}(\varphi)$ is affine because $\mathscr{L}_{n}(\widetilde{X})$ and $\mathscr{L}_{n}(X)$ are affine, so

$$
C_{n}^{\widetilde{w}}(\varphi) /\left(\mathscr{L}_{n}\left(G^{\prime}\right) \otimes_{k} k^{\prime}\right) \longrightarrow\left[C_{n}^{\widetilde{w}}(\varphi) /\left(\mathscr{L}_{n}(G) \otimes_{k} k^{\prime}\right)\right]
$$

is an $\left(\mathscr{L}_{n}\left(T^{\prime}\right) \otimes_{k} k^{\prime}\right)$-torsor and the source is an affine scheme. By Theorem 4.9, there exists some $\psi \in\left(\mathscr{L}(\pi)^{-1}(\varphi)\right)\left(k^{\prime}\right)$ with $\operatorname{trop}(\psi)=\widetilde{w}$, so

$$
\theta_{n}(\psi) \in\left[C_{n}^{\widetilde{w}}(\varphi) /\left(\mathscr{L}_{n}(G) \otimes_{k} k^{\prime}\right)\right]\left(k^{\prime}\right) .
$$

Since $\mathscr{L}_{n}\left(T^{\prime}\right)$ is a special group by Remark 2.4, $\theta_{n}(\psi)$ lifts to a $k^{\prime}$-point of $C_{n}^{\widetilde{w}}(\varphi) /\left(\mathscr{L}_{n}\left(G^{\prime}\right) \otimes_{k} k^{\prime}\right)$.

Next, by Proposition 5.13(2)-(4), the group $\left(G_{n}^{w} / \mathscr{L}_{n}\left(G^{\prime}\right)\right) \otimes_{k} k^{\prime}$ acts transitively on $C_{n}^{\widetilde{w}}(\varphi) /\left(\mathscr{L}_{n}\left(G^{\prime}\right) \otimes_{k} k^{\prime}\right)$, and each $k^{\prime}$-point has stabiliser $\left(H_{n}^{\widetilde{w}} /\left(H_{n}^{\widetilde{w}} \cap \mathscr{L}_{n}\left(G^{\prime}\right)\right)\right) \otimes_{k} k^{\prime}$, so [DG70, Chapter III, Section 3, Proposition 5.2] gives an $\left(\mathscr{L}_{n}\left(T^{\prime}\right) \otimes_{k} k^{\prime}\right)$-equivariant isomorphism

$$
\begin{aligned}
C_{n}^{\widetilde{w}}(\varphi) /\left(\mathscr{L}_{n}\left(G^{\prime}\right) \otimes_{k} k^{\prime}\right) & \cong\left(C_{n}^{\widetilde{w}}(\varphi) /\left(\mathscr{L}_{n}\left(G^{\prime}\right) \otimes_{k} k^{\prime}\right)\right)_{\text {red }} \\
& \cong\left(\left(G_{n}^{w} / \mathscr{L}_{n}\left(G^{\prime}\right)\right) /\left(H_{n}^{\widetilde{w}} /\left(H_{n}^{\widetilde{w}} \cap \mathscr{L}_{n}\left(G^{\prime}\right)\right)\right)\right) \otimes_{k} k^{\prime} \\
& \cong\left[\left(G_{n}^{w} / H_{n}^{\widetilde{w}}\right) / \mathscr{L}_{n}\left(G^{\prime}\right)\right] \otimes_{k} k^{\prime}
\end{aligned}
$$


where the last isomorphism holds because $\mathscr{L}_{n}\left(G^{\prime}\right) \cap H_{n}^{\widetilde{w}}=\{1\}$. Taking the quotient by $\mathscr{L}_{n}\left(T^{\prime}\right) \otimes_{k} k^{\prime}$, we obtain

$$
\left[C_{n}^{\widetilde{w}}(\varphi) /\left(\mathscr{L}_{n}(G) \otimes_{k} k^{\prime}\right)\right] \cong\left[\left(G_{n}^{w} / H_{n}^{\widetilde{w}}\right) / \mathscr{L}_{n}(G)\right] \otimes_{k} k^{\prime} \cong\left[\left(G_{n}^{w} / \mathscr{L}_{n}(G)\right) / H_{n}^{\widetilde{w}}\right] \otimes_{k} k^{\prime} .
$$

We may now complete the proof of Theorem 5.1.

Proof of Theorem 5.1. Set $w \in \sigma \cap N$, let $f_{1}, \ldots, f_{r}$ be the minimal generators of $F$, let $n_{w}^{\prime}$ and $j_{w}^{\prime}$ be as in the statement of Proposition 5.7 and let $n_{2}$ be as in the statement of Proposition 5.15. Recalling that $\beta^{-1}(w)$ is a finite set by Proposition 2.15 , set

$$
n_{w}=\max \left\{n_{w}^{\prime}, n_{2}, 2\left\langle\widetilde{w}, f_{i}\right\rangle-1 \mid i \in\{1, \ldots, r\}, \widetilde{w} \in \beta^{-1}(w)\right\}
$$

and

$$
\Theta_{w}=\sum_{\widetilde{w} \in \beta^{-1}(w)} \mathbb{L}^{j_{w}^{\prime}-\sum_{i=1}^{r}\left\langle\widetilde{w}, f_{i}\right\rangle} \in K_{0}\left(\mathbf{S t a c k}_{k}\right),
$$

and for each $n \geq n_{w}$, set

$$
\mathcal{F}_{n}=\bigsqcup_{\widetilde{w} \in \beta^{-1}(w)}\left[\left(G_{n}^{w} / \mathscr{L}_{n}(G)\right) / H_{n}^{\widetilde{w}}\right] .
$$

We now finish proving each part of Theorem 5.1 separately.

1. For all $n \geq n_{w}$,

$$
\begin{aligned}
\mathrm{e}\left(\mathcal{F}_{n}\right) & =\sum_{\widetilde{w} \in \beta^{-1}(w)} \mathrm{e}\left(\left[\left(G_{n}^{w} / \mathscr{L}_{n}(G)\right) / H_{n}^{\widetilde{w}}\right]\right) \\
& =\sum_{\widetilde{w} \in \beta^{-1}(w)} \mathrm{e}\left(G_{n}^{w} / \mathscr{L}_{n}(G)\right) \mathbb{L}^{-\sum_{i=1}^{r}\left\langle\widetilde{w}, f_{i}\right\rangle}=\sum_{\widetilde{w} \in \beta^{-1}(w)} \mathbb{L}^{j_{w}^{\prime}-\sum_{i=1}^{r}\left\langle\widetilde{w}, f_{i}\right\rangle}=\Theta_{w},
\end{aligned}
$$

where the second equality follows from Proposition 5.5 and the fact that $\mathbb{G}_{a}$ is a special group, and the third equality follows from Proposition 5.7.

2. This is Proposition 5.15 - that is, it follows from our choice of $n_{2}$ and each $\mathcal{F}_{n}$.

\section{Gorenstein measure and toric varieties}

Set $d \in \mathbb{N}$, let $N \cong \mathbb{Z}^{d}$ be a lattice, let $T=\operatorname{Spec}\left(k\left[N^{*}\right]\right)$ be the algebraic torus with cocharacter lattice $N$, let $\sigma$ be a pointed rational cone in $N_{\mathbb{R}}$ and let $X$ be the affine $T$-toric variety associated to $\sigma$. We assume that $X$ is $\mathbb{Q}$-Gorenstein and let $m \in \mathbb{Z}_{>0}$ and $q \in N^{*}$ be such that if $v$ is the first lattice point of any ray of $\sigma$,

$$
\langle v, q\rangle=m \text {. }
$$

Then $m K_{X}$ is Cartier, so we have the ideal sheaf $\mathscr{I}_{X, m}$ on $X$. Also note that any $\mathbb{Q}$-Gorenstein toric variety has log-terminal singularities [Bat98, Corollary 4.2], so the Gorenstein measure $\mu_{X}^{\text {Gor }}$ is well defined.

In this section, we prove Theorem 6.1 about the Gorenstein measure $\mu_{X}^{\text {Gor }}$. In section 8 , we will use this theorem and Theorem 7.1 to compare $\mu_{X}^{\text {Gor }}$ with the motivic measure $\mu_{\mathcal{X}}$ of the canonical stack $\mathcal{X}$ over $X$. 
Although we will only use Theorem 6.1 in the case where $\sigma$ is $d$-dimensional, there is no need to make that assumption on $\sigma$ in this section.

Theorem 6.1. Set $w \in \sigma \cap N \subset \operatorname{Hom}\left(\sigma^{\vee} \cap N^{*}, \mathbb{N} \cup\{\infty\}\right)$.

(a) The restriction of $\operatorname{ord}_{\mathcal{F}_{X, m}}$ to $\operatorname{trop}^{-1}(w) \subset \mathscr{L}(X)$ is constant and not equal to infinity. In particular, there exists some $j_{w} \in \mathbb{Z}$ such that for any measurable subset $C \subset \operatorname{trop}^{-1}(w) \subset \mathscr{L}(X)$,

$$
\mu_{X}^{\text {Gor }}(C)=\left(\mathbb{L}^{1 / m}\right)^{j_{w}} \mu_{X}(C) \in \widehat{\mathscr{M}}_{k}\left[\mathbb{L}^{1 / m}\right] .
$$

(b) The set $\operatorname{trop}^{-1}(w) \subset \mathscr{L}(X)$ is measurable and

$$
\mu_{X}^{\mathrm{Gor}}\left(\operatorname{trop}^{-1}(w)\right)=\mathbb{L}^{-d}(\mathbb{L}-1)^{d}\left(\mathbb{L}^{1 / m}\right)^{-\langle w, q\rangle} \in \widehat{\mathbb{M}}_{k}\left[\mathbb{L}^{1 / m}\right] .
$$

Remark 6.2. Summing over $w \in \sigma \cap N$, Theorem 6.1(b) gives Batyrev's formula [Bat98, Theorem 4.3] for the stringy Hodge-Deligne invariant of a toric variety. Furthermore, Theorem 6.1(b) appears to be a special case of [BM13, Lemma 4.5]. For the benefit of the reader, we include a short self-contained proof.

Remark 6.3. When $w$ is an integer combination of lattice points on the rays of $\sigma$, we have that $\langle w, q\rangle$ is divisible by $m$, so in that case Theorem 6.1(b) implies

$$
\mu_{X}^{\mathrm{Gor}}\left(\operatorname{trop}^{-1}(w)\right) \in \widehat{\mathscr{M}}_{k}
$$

\subsection{Gorenstein measure and monomial ideals}

For the remainder of this section, let $M=N^{*}$, let $P=\sigma^{\vee} \cap M$, and for each $p \in P$, let $\chi^{p} \in k[P]$ be the monomial indexed by $p$.

If $\mathscr{J}$ is a nonzero ideal sheaf on $X$ generated by monomials $\left\{\chi^{p_{i}}\right\}_{i}$, then for any $\varphi \in \mathscr{L}(X)$ with $\operatorname{trop}(\varphi)=w$,

$$
\operatorname{ord}_{\mathcal{J}}(\varphi)=\min _{i}\left\langle w, p_{i}\right\rangle \in \mathbb{Z}_{\geq 0}
$$

Therefore, to prove Theorem 6.1(a) it is sufficient to show that the ideal $\mathscr{F}_{X, m}$ is generated by monomials.

For the remainder of this subsection, fix a basis $e_{1}, \ldots, e_{d}$ for $M$, and for any $p_{1}, \ldots, p_{d} \in P$, set

$$
c\left(p_{1}, \ldots, p_{d}\right)=\operatorname{det}\left(\left(a_{i, j}\right)_{i, j}\right) \in \mathbb{Z}
$$

where the $a_{i, j} \in \mathbb{Z}$ are such that $p_{j}=\sum_{i=1}^{d} a_{i, j} e_{i}$ for all $j \in\{1, \ldots, d\}$. For any $\left(p_{i, j}\right)_{i \in\{1, \ldots, m\}, j \in\{1, \ldots, d\}} \in P^{m d}$, set

$$
z\left(\left(p_{i, j}\right)_{i, j}\right)=\chi^{-q} \prod_{i=1}^{m} c\left(p_{i, 1}, \ldots, p_{i, d}\right) \chi^{p_{i, 1}+\cdots+p_{i, d}} \in k[M] .
$$

Lemma 6.4. Set $\left(p_{i, j}\right)_{i, j} \in P^{m d}$. Then

$$
z\left(\left(p_{i, j}\right)_{i, j}\right) \in k[P]
$$


Proof. If $-q+\sum_{i=1}^{m} \sum_{j=1}^{d} p_{i, j} \in P$, we are done, so we may assume that there exists some first lattice point $v$ of a ray of $\sigma$ such that $\left\langle v,-q+\sum_{i=1}^{m} \sum_{j=1}^{d} p_{i, j}\right\rangle<0$. Then

$$
0>\left\langle v,-q+\sum_{i=1}^{m} \sum_{j=1}^{d} p_{i, j}\right\rangle=\sum_{i=1}^{m}\left\langle v,-\frac{1}{m} q+\sum_{j=1}^{d} p_{i, j}\right\rangle=\sum_{i=1}^{m}\left(-1+\sum_{j=1}^{d}\left\langle v, p_{i, j}\right\rangle\right),
$$

so for some $i \in\{1, \ldots, m\}$, we have $\left\langle v, p_{i, j}\right\rangle=0$ for all $j \in\{1, \ldots, d\}$, so

$$
c\left(p_{i, 1}, \ldots, p_{i, d}\right)=0,
$$

which implies that $z\left(\left(p_{i, j}\right)_{i, j}\right)=0 \in k[P]$.

We now prove the next proposition, which as discussed, immediately implies Theorem 6.1(a). Note that because $\sigma$ is pointed, $P^{\mathrm{gp}}=M$, so the $c\left(p_{1}, \ldots, p_{d}\right)$ are not all equal to 0 and the $z\left(\left(p_{i, j}\right)_{i, j}\right)$ are not all equal to 0 .

Proposition 6.5. The ideal $\mathcal{F}_{X, m}$ is generated by the set

$$
\left\{z\left(\left(p_{i, j}\right)_{i, j}\right) \mid\left(p_{i, j}\right)_{i, j} \in P^{m d}\right\}
$$

Proof. Since $k[P]$ is generated over $k$ by the set $\left\{\chi^{p} \mid p \in P\right\}$, we see that $\Gamma\left(X, \Omega_{X}^{d}\right)$ is generated by the elements $\chi^{p_{1}} \wedge \cdots \wedge \chi^{p_{d}}$ as $p_{1}, \ldots, p_{d}$ range over elements of $P$. So $\Gamma\left(X,\left(\Omega_{X}^{d}\right)^{\otimes m}\right)$ is generated by the set

$$
\left\{\bigotimes_{i=1}^{m} \mathrm{~d} \chi^{p_{i, 1}} \wedge \cdots \wedge \mathrm{d} \chi^{p_{i, d}} \mid\left(p_{i, j}\right)_{i, j} \in P^{m d}\right\}
$$

We next show that the global sections of $\omega_{X, m}$ are generated by

$$
\chi^{q} \cdot\left(\mathrm{d} \log \chi^{e_{1}} \wedge \cdots \wedge \mathrm{d} \log \chi^{e_{d}}\right)^{\otimes m}
$$

this is essentially given by [CLS11, Proposition 8.2.9], but they only state the result for $\omega_{X}$ instead of $\omega_{X, m}$. The proof for all $m$ works identically. To see this, let $\iota: X_{\mathrm{sm}} \hookrightarrow X$ be the inclusion of the smooth locus. By [CLS11, Theorem 8.2.3],

$$
\omega_{X, m}=\iota_{*}\left(\left(\Omega_{X_{\mathrm{sm}}}^{\operatorname{dim} X}\right)^{\otimes m}\right) \cong \iota_{*} \mathcal{O}_{X_{\mathrm{sm}}}\left(-m \sum_{\rho} D_{\rho}\right)=\mathcal{O}_{X}\left(-m \sum_{\rho} D_{\rho}\right),
$$

where the sum runs over all $\rho \in \Sigma(1)$ and $D_{\rho}$ denotes the corresponding torus-invariant divisor. Implicit in [CLS11, Theorem 8.2.3] is an identification of $\Omega_{X_{\mathrm{sm}}}^{\operatorname{dim} X}$ with a subsheaf of $\mathcal{O}_{X_{\mathrm{sm}}}$; this identification comes from [CLS11, Maps (8.1.3) and (8.1.5)] and can be described as follows. We have an inclusion of $\Omega_{X_{\mathrm{sm}}}^{\operatorname{dim} X}$ into the logarithmic differentials $\Omega_{X_{\mathrm{sm}}}^{\operatorname{dim} X}(\log D)$, and the latter is isomorphic to $\mathcal{O}_{X_{\mathrm{sm}}}$ via the map

$$
\begin{gathered}
\mathcal{O}_{X_{\mathrm{sm}}} \stackrel{\cong}{\rightarrow} \Omega_{X_{\mathrm{sm}}}^{\operatorname{dim} X}(\log D), \\
f \mapsto f \cdot \mathrm{d} \log \chi^{e_{1}} \wedge \cdots \wedge \mathrm{d} \log \chi^{e_{d}} .
\end{gathered}
$$

For each $\rho \in \Sigma(1)$, let $v_{\rho}$ denote the first lattice point on the ray $\rho$. Having established that $\omega_{X, m} \cong$ $\mathcal{O}_{X}\left(-m \sum_{\rho} D_{\rho}\right)$, [CLS11, Proposition 4.3.2] tells us that $\Gamma\left(X, \omega_{X, m}\right)$ is generated over $k$ by the sections 
of the form $\chi^{p} \cdot\left(\mathrm{d} \log \chi^{e_{1}} \wedge \cdots \wedge \mathrm{d} \log \chi^{e_{d}}\right)^{\otimes m}$ for $p \in M$ such that $\left\langle p, v_{\rho}\right\rangle \geq m$ for all $\rho \in \Sigma(1)$. This condition on the inner product implies $p-q \in P$, and so $\Gamma\left(X, \omega_{X, m}\right)$ is generated over $k[P]$ by

$$
\chi^{q} \cdot\left(\mathrm{d} \log \chi^{e_{1}} \wedge \cdots \wedge \mathrm{d} \log \chi^{e_{d}}\right)^{\otimes m} .
$$

Lastly, for any $p_{1}, \ldots, p_{d} \in P$,

$$
\begin{aligned}
\mathrm{d} \chi^{p_{1}} \wedge \cdots \wedge \mathrm{d} \chi^{p_{d}} & =\chi^{p_{1}+\cdots+p_{d}} \cdot \mathrm{d} \log \chi^{p_{1}} \wedge \cdots \wedge \mathrm{d} \log \chi^{p_{d}} \\
& =c\left(p_{1}, \ldots, p_{d}\right) \chi^{p_{1}+\cdots+p_{d}} \cdot \mathrm{d} \log \chi^{e_{1}} \wedge \cdots \wedge \mathrm{d} \log \chi^{e_{d}} .
\end{aligned}
$$

Thus for any $\left(p_{i, j}\right)_{i, j} \in P^{m d}$,

$$
\bigotimes_{i=1}^{m} \mathrm{~d} \chi^{p_{i, 1}} \wedge \cdots \wedge \mathrm{d} \chi^{p_{i, d}}=z\left(\left(p_{i, j}\right)_{i, j}\right) \cdot \chi^{q} \cdot\left(\mathrm{d} \log \chi^{e_{1}} \wedge \cdots \wedge \mathrm{d} \log \chi^{e_{d}}\right)^{\otimes m} .
$$

The proposition then follows from the definition of $\mathscr{J}_{X, m}$.

Remark 6.6. Proposition 6.5 implies that if $\varphi \in \mathscr{L}(X)$ with $\operatorname{trop}(\varphi)=w$, then

$$
\operatorname{ord}_{\mathscr{F}_{X, m}}(\varphi)=-\langle w, q\rangle+\min _{\substack{p_{1}, \ldots, p_{d} \in P \\ c\left(p_{1}, \ldots, p_{d}\right) \neq 0}} m\left\langle w, p_{1}+\cdots+p_{d}\right\rangle
$$

so if $w$ is an integer combination of lattice points on the rays of $\sigma$, then $\operatorname{ord}_{\mathscr{f}_{X, m}}(\varphi)$ is divisible by $m$ and $\mu_{X}^{\text {Gor }}(C) \in \widehat{\mathscr{M}}_{k}$ for any measurable subset $C \subset \operatorname{trop}^{-1}(w)$.

\subsection{Gorenstein measure and toric modifications}

In this subsection, we complete the proof of Theorem 6.1(b). We first handle the case where $w=0$.

Proposition 6.7. We have

$$
\mu_{X}^{\text {Gor }}\left(\operatorname{trop}^{-1}(0)\right)=\mathbb{L}^{-d}(\mathbb{L}-1)^{d}
$$

Proof. If $\varphi \in \mathscr{L}(X)$, then $\operatorname{trop}(\varphi)=0$ if and only if $\varphi^{*}(p)$ is a unit for all $p \in P$, which occurs if and only if $\varphi \in \mathscr{L}(T)$. Thus

$$
\operatorname{trop}^{-1}(0)=\mathscr{L}(T) \subset \mathscr{L}(X),
$$

and because $T$ is smooth, we have

$$
\mu_{X}^{\mathrm{Gor}}\left(\operatorname{trop}^{-1}(0)\right)=\mu_{T}(\mathscr{L}(T))=\mathbb{L}^{-\operatorname{dim} T} \mathrm{e}(T)=\mathbb{L}^{-d}(\mathbb{L}-1)^{d},
$$

where the first equality is given by Theorem 6.1(a).

We now only need to prove Theorem 6.1(b) in the case where $w \neq 0$. For the remainder of this section, fix $w \in \sigma \cap N$, assume that $w \neq 0$, and let $\ell \in \mathbb{Z}_{>0}$ be such that $(1 / \ell) w$ is the first lattice point of the ray $\tau:=\mathbb{R}_{\geq 0} w$. We will compute $\mu_{X}\left(\operatorname{trop}^{-1}(w)\right)$ by applying the change-of-variables formula to a certain toric modification of $X$. Let $Y \cong \mathbb{A}^{1} \times \mathbb{G}_{m}^{d-1}$ be the affine $T$-toric variety whose fan is given by $\tau$, let $D$ be the (irreducible) boundary divisor of $Y$ and let $\rho: Y \rightarrow X$ be the toric morphism induced 
by the identity map $N \rightarrow N$. It is standard to compute the relative canonical divisor of such a birational toric morphism. In this case,

$$
m K_{Y}-\rho^{*}\left(m K_{X}\right)=\left(\frac{1}{\ell}\langle w, q\rangle-m\right) D .
$$

For the remainder of this section, let $\mathcal{O}(-D)$ be the ideal sheaf of $D$ in $Y$.

Proposition 6.8. The map $\mathscr{L}(\rho): \mathscr{L}(Y) \rightarrow \mathscr{L}(X)$ induces a bijection

$$
\left(\operatorname{ord}_{\mathcal{O}(-D)}^{-1}(\ell)\right)\left(k^{\prime}\right) \rightarrow\left(\operatorname{trop}^{-1}(w)\right)\left(k^{\prime}\right)
$$

for every field extension $k^{\prime}$ of $k$.

Proof. Let $k^{\prime}$ be a field extension of $k$. By construction, $\mathscr{L}(\rho)$ induces a bijection $(\mathscr{L}(Y) \backslash \mathscr{L}(Y \backslash$ $T))\left(k^{\prime}\right) \rightarrow\left(\operatorname{trop}^{-1}\left(N \cap \mathbb{R}_{\geq 0} w\right)\right)\left(k^{\prime}\right)$. Therefore it is sufficient to show that

$$
\mathscr{L}(\rho)^{-1}\left(\left(\operatorname{trop}^{-1}(w)\right)\left(k^{\prime}\right)\right)=\left(\operatorname{ord}_{\mathcal{O}(-D)}^{-1}(\ell)\right)\left(k^{\prime}\right) .
$$

By Lemma 4.7(2), it is enough to show that if $u \in \tau^{\vee} \cap M$, there exists $u^{\prime} \in \tau^{\vee} \cap M$ with $u+u^{\prime} \in \sigma^{\vee} \cap M$. Consider the quotient map $\eta: M \rightarrow M /\left(w^{\perp} \cap M\right) \cong \mathbb{Z}$. Since $\sigma^{\vee} \subset \tau^{\vee}$, we see that $\eta\left(\sigma^{\vee} \cap M\right) \subset$ $\eta\left(\tau^{\vee} \cap M\right)=\mathbb{N}$. So for any $u \in \tau^{\vee} \cap M$, there exists $n \in \mathbb{Z}_{>0}$ such that $n \eta(u) \in \eta\left(\sigma^{\vee} \cap M\right)-$ that is, for some choice of $u^{\prime \prime} \in w^{\perp} \cap M$, letting $u^{\prime}=u^{\prime \prime}+(n-1) u$, we have $u+u^{\prime} \in \sigma^{\vee} \cap M$.

The next proposition completes the proof of Theorem 6.1(b).

Proposition 6.9. We have

$$
\mu_{X}^{\mathrm{Gor}}\left(\operatorname{trop}^{-1}(w)\right)=\mathbb{L}^{-d}(\mathbb{L}-1)^{d}\left(\mathbb{L}^{1 / m}\right)^{-\langle w, q\rangle} .
$$

Proof. By Theorem 6.1(a), there exists some $j_{w} \in \mathbb{Z}$ such that $\operatorname{ord}_{\mathcal{F}_{X, m}}$ is equal to $j_{w}$ on $\operatorname{trop}^{-1}(w)$.

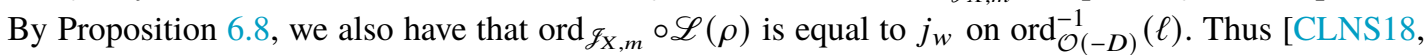
Chapter 7, Proposition 3.2.5] implies that on $\operatorname{ord}_{\mathcal{O}(-D)}^{-1}(\ell)$,

$$
-\operatorname{ordjac}_{\rho}=-\frac{1}{m} \operatorname{ord}_{\mathscr{J}_{X, m}} \circ \mathscr{L}(\rho)-\frac{1}{m}\left(\frac{1}{\ell}\langle w, q\rangle-m\right) \operatorname{ord}_{\mathcal{O}(-D)}=-\frac{j_{w}}{m}-\frac{1}{m}\langle w, q\rangle+\ell,
$$

where $\operatorname{ordjac}_{\rho}: \mathscr{L}(Y) \rightarrow \mathbb{N} \cup\{\infty\}$ denotes the order function of the Jacobian ideal of $\rho$. Therefore,

$$
\begin{aligned}
& \mu_{X}^{\text {Gor }}\left(\operatorname{trop}^{-1}(w)\right)=\int_{\operatorname{trop}^{-1}(w)}\left(\mathbb{L}^{1 / m}\right)^{\text {ord } f_{X, m}} \mathrm{~d} \mu_{X}=\left(\mathbb{L}^{1 / m}\right)^{j_{w}} \mu_{X}\left(\operatorname{trop}^{-1}(w)\right)
\end{aligned}
$$

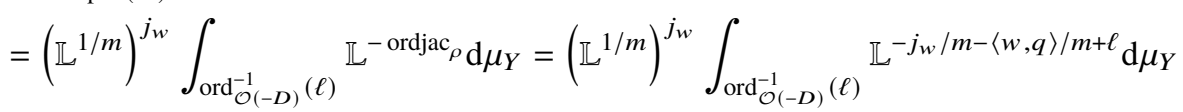

$$
\begin{aligned}
& =\left(\mathbb{L}^{1 / m}\right)^{-\langle w, q\rangle} \mathbb{L}^{\ell} \mu_{Y}\left(\operatorname{ord}_{\mathcal{O}(-D)}^{-1}(\ell)\right)=\mathbb{L}^{-d}(\mathbb{L}-1)^{d}\left(\mathbb{L}^{1 / m}\right)^{-\langle w, q\rangle},
\end{aligned}
$$

where the third equality is due to Proposition 6.8 and the motivic change-of-variables formula (see, for example, [CLNS18, Chapter 6, Theorem 4.3.1]), and the final equality follows from

$$
\mu_{Y}\left(\operatorname{ord}_{\mathcal{O}(-D)}^{-1}(\ell)\right)=\mathrm{e}(D)(\mathbb{L}-1) \mathbb{L}^{-d-\ell}=(\mathbb{L}-1)^{d} \mathbb{L}^{-d-\ell},
$$

which is a consequence of [CLNS18, Chapter 7, Lemma 3.3.3]. 


\section{Motivic measure and canonical stacks}

Set $d \in \mathbb{N}$, let $N \cong \mathbb{Z}^{d}$ be a lattice, let $T=\operatorname{Spec}\left(k\left[N^{*}\right]\right)$ be the algebraic torus with cocharacter lattice $N$, let $\sigma$ be a pointed rational cone in $N_{\mathbb{R}}$, let $X$ be the affine $T$-toric variety associated to $\sigma$, let $\mathcal{X}$ be the canonical stack over $X$ and let $\pi: \mathcal{X} \rightarrow X$ be the canonical map. We assume that $\sigma$ is $d$-dimensional and use the notation listed in Notation 2.19. We assume that $X$ is $\mathbb{Q}$-Gorenstein and let $m \in \mathbb{Z}_{>0}$ and $q \in P$ be such that if $v$ is the first lattice point of any ray of $\sigma$,

$$
\langle v, q\rangle=m
$$

In this section, we prove the following theorem about the motivic measure $\mu_{\mathcal{X}}$, which mirrors Theorem 6.1 for $\mu_{X}^{\text {Gor }}$. In section 8, we will combine these two theorems to compare the measures $\mu_{\mathcal{X}}$ and $\mu_{X}^{\text {Gor }}$.

Theorem 7.1. Set $w \in \sigma \cap N \subset \operatorname{Hom}(P, \mathbb{N} \cup\{\infty\})$.

(a) If $C \subset \operatorname{trop}^{-1}(w) \subset \mathscr{L}(X)$ is measurable, then $\mathscr{L}(\pi)^{-1}(C)$ is a measurable subset of $|\mathscr{L}(\mathcal{X})|$. Furthermore, there exists $\Theta_{w} \in \widehat{M}_{k}$ such that for any measurable subset $C \subset \operatorname{trop}^{-1}(w) \subset \mathscr{L}(X)$,

$$
\mu_{\mathcal{X}}\left(\mathscr{L}(\pi)^{-1}(C)\right)=\Theta_{w} \mu_{X}(C) .
$$

(b) The set $\mathscr{L}(\pi)^{-1}\left(\operatorname{trop}^{-1}(w)\right) \subset|\mathscr{L}(\mathcal{X})|$ is measurable and

$$
\mu_{\mathcal{X}}\left(\mathscr{L}(\pi)^{-1}\left(\operatorname{trop}^{-1}(w)\right)\right)=\left(\# \beta^{-1}(w)\right) \mathbb{L}^{-d}(\mathbb{L}-1)^{d}\left(\mathbb{L}^{1 / m}\right)^{-\langle w, q\rangle} .
$$

Remark 7.2. A priori, we only have that

$$
\left(\# \beta^{-1}(w)\right) \mathbb{L}^{-d}(\mathbb{L}-1)^{d}\left(\mathbb{L}^{1 / m}\right)^{-\langle w, q\rangle} \in \widehat{\mathscr{M}}_{k}\left[\mathbb{L}^{1 / m}\right] \supset \widehat{\mathscr{M}_{k}}
$$

but by Lemma 7.9 we have either that $\beta^{-1}(w)=\emptyset$ or that $\langle w, q\rangle$ is divisible by $m$, so

$$
\left(\# \beta^{-1}(w)\right) \mathbb{L}^{-d}(\mathbb{L}-1)^{d}\left(\mathbb{L}^{1 / m}\right)^{-\langle w, q\rangle} \in \widehat{\mathscr{M}}_{k} .
$$

Before we prove Theorem 7.1, we show that it implies the next proposition.

Proposition 7.3. Let $C \subset \mathscr{L}(X)$ be a measurable subset. Then $\mathscr{L}(\pi)^{-1}(C)$ is a measurable subset of $|\mathscr{L}(\mathcal{X})|$ and

$$
\mu_{\mathcal{X}}\left(\mathscr{L}(\pi)^{-1}(C)\right)=\sum_{w \in \sigma \cap N} \mu_{\mathcal{X}}\left(\mathscr{L}(\pi)^{-1}\left(\operatorname{trop}^{-1}(w) \cap C\right)\right)
$$

Remark 7.4. By Remark 4.6 and Theorem $7.1(\mathrm{a})$, each $\mathscr{L}(\pi)^{-1}\left(\operatorname{trop}^{-1}(w) \cap C\right)$ in the statement of Proposition 7.3 is a measurable subset of $|\mathscr{L}(\mathcal{X})|$.

Proof. Set

$$
\begin{aligned}
\mathcal{C} & =\mathscr{L}(\pi)^{-1}(C), \\
\mathcal{C}^{(0)} & =\bigcup_{w \in \sigma \cap N} \mathscr{L}(\pi)^{-1}\left(\operatorname{trop}^{-1}(w) \cap C\right), \\
\mathcal{C}^{(\infty)} & =\mathcal{C} \backslash \mathcal{C}^{(0)} .
\end{aligned}
$$


For each $w \in \sigma \cap N$,

$$
\begin{aligned}
\left\|\mu_{\mathcal{X}}\left(\mathscr{L}(\pi)^{-1}\left(\operatorname{trop}^{-1}(w) \cap C\right)\right)\right\| & \leq\left\|\mu_{\mathcal{X}}\left(\mathscr{L}(\pi)^{-1}\left(\operatorname{trop}^{-1}(w)\right)\right)\right\| \\
& \leq\left\|\mathbb{L}^{-d}(\mathbb{L}-1)^{d}\right\| \exp (-\langle w, q\rangle / m),
\end{aligned}
$$

where the first inequality is by Proposition 3.29 and the second is by Theorem 7.1(b). For each $\varepsilon \in \mathbb{R}_{>0}$ there are only finitely many $w \in \sigma \cap N$ with $\exp (-\langle w, q\rangle / m) \geq \varepsilon$, so Proposition 3.27 implies that $\mathcal{C}^{(0)}$ is measurable and

$$
\mu_{\mathcal{X}}\left(\mathcal{C}^{(0)}\right)=\sum_{w \in \sigma \cap N} \mu_{\mathcal{X}}\left(\mathscr{L}(\pi)^{-1}\left(\operatorname{trop}^{-1}(w) \cap C\right)\right)
$$

By Proposition 3.28 and Proposition 3.30, the $\operatorname{set} \mathcal{C}^{(\infty)}$ is measurable and

$$
\mu_{\mathcal{X}}\left(\mathcal{C}^{(\infty)}\right)=0
$$

Therefore by Proposition 3.27, the set $\mathcal{C}=\mathcal{C}^{(0)} \sqcup \mathcal{C}^{(\infty)}$ is measurable and

$$
\mu_{\mathcal{X}}(\mathcal{C})=\mu_{\mathcal{X}}\left(\mathcal{C}^{(0)}\right)=\sum_{w \in \sigma \cap N} \mu_{\mathcal{X}}\left(\mathscr{L}(\pi)^{-1}\left(\operatorname{trop}^{-1}(w) \cap C\right)\right) .
$$

We will use the remainder of this section to prove Theorem 7.1.

\subsection{Canonical stacks and preimages of measurable subsets}

In this subsection, we will prove Theorem 7.1(a). We begin with a couple lemmas.

Lemma 7.5. Let $Y$ be an irreducible finite-type scheme over $k$ with smooth locus $Y_{\mathrm{sm}} \subset Y$, and let $C \subset \mathscr{L}(Y)$ be a cylinder such that $C \cap \mathscr{L}\left(Y \backslash Y_{\mathrm{sm}}\right)=\emptyset$.

Then there exists some $n_{C} \in \mathbb{N}$ that satisfies the following: For any field extension $k^{\prime}$ of $k$, any $n \geq n_{C}$ and any $\varphi_{n} \in \mathscr{L}_{n}(Y)\left(k^{\prime}\right)$ with image in $\theta_{n}(C)$, there exists some $\varphi \in \mathscr{L}(Y)\left(k^{\prime}\right)$ with image in $C$ such that $\theta_{n}(\varphi)=\varphi_{n}$.

Proof. By [CLNS18, Chapter 5, Propositions 1.3.2(a) and 2.3.4], there exists a function ordjac $Y$ : $\mathscr{L}(Y) \rightarrow \mathbb{N} \cup\{\infty\}$ and some $c \in \mathbb{Z}_{>0}$ such that

○ for every $n \in \mathbb{N}$, the set $\operatorname{ordjac}_{Y}^{-1}(n) \subset \mathscr{L}(Y)$ is a cylinder;

o the image of $\mathscr{L}(Y) \backslash \mathscr{L}\left(Y \backslash Y_{\text {sm }}\right)$ under ordjac ${ }_{Y}$ is contained in $\mathbb{N}$; and

○ for every $n \in \mathbb{N}$, field extension $k^{\prime}$ of $k$ and $\varphi_{n} \in \mathscr{L}_{n}(Y)\left(k^{\prime}\right)$ whose image in $\mathscr{L}_{n}(Y)$ is contained in $\theta_{n}\left(\operatorname{ordjac}_{Y}^{-1}\left(n^{\prime}\right)\right)$ for some $n^{\prime} \leq n / c$, there exists some $\varphi \in \mathscr{L}(Y)\left(k^{\prime}\right)$ with $\theta_{n}(\varphi)=\varphi_{n}$.

Because $C \cap \mathscr{L}\left(Y \backslash Y_{\mathrm{sm}}\right)=\emptyset$, the collection $\left\{\operatorname{ordjac}_{Y}^{-1}(n)\right\}_{n \in \mathbb{N}}$ is a cover of the cylinder $C$ by cylinders. Thus by the quasicompactness of the constructible topology of $\mathscr{L}(Y)$ (see, for example, [CLNS18, Appendix, Theorem 1.2.4(a)]), there exists some $n_{C}^{\prime} \in \mathbb{N}$ such that $C \subset \bigcup_{n=0}^{n_{C}^{\prime}} \operatorname{ordjac}_{Y}^{-1}(n)$. Let $n_{C} \in \mathbb{N}$ be such that $n_{C} \geq c n_{C}^{\prime}$ and such that $C$ is the preimage under $\theta_{n_{C}}$ of a constructible subset of $\mathscr{L}_{n_{C}}(Y)$.

Now let $k^{\prime}$ be a field extension of $k$, let $n \geq n_{C}$ and let $\varphi_{n} \in \mathscr{L}_{n}(Y)\left(k^{\prime}\right)$ have image in $\theta_{n}(C)$. Then the image of $\varphi_{n}$ is contained in $\theta_{n}\left(\operatorname{ordjac}_{Y}^{-1}\left(n^{\prime}\right)\right)$ for some $n^{\prime} \leq n_{C}^{\prime} \leq n_{C} / c \leq n / c$, so there exists some $\varphi \in \mathscr{L}(Y)\left(k^{\prime}\right)$ with $\theta_{n}(\varphi)=\varphi_{n}$. Because $C$ is the preimage of a subset of $\mathscr{L}_{n}(Y)$, the arc $\varphi$ has image in $C$.

Lemma 7.6. Let $Y$ be a finite-type scheme over $k$, let $\mathcal{Y}$ be a smooth Artin stack over $k$, let $\xi: \mathcal{Y} \rightarrow Y$ be a morphism, let $C \subset \mathscr{L}(Y)$ be a cylinder and set $\mathcal{C}=\mathscr{L}(\xi)^{-1}(C) \subset|\mathscr{L}(\mathcal{Y})|$. 
Then $\mathcal{C}$ is a cylinder and there exists some $n_{0} \in \mathbb{N}$ such that for all $n \geq n_{0}$,

$$
\theta_{n}(\mathcal{C})=\mathscr{L}_{n}(\xi)^{-1}\left(\theta_{n}(C)\right)
$$

Proof. We first note that for all $n \in \mathbb{N}$, we have an obvious inclusion

$$
\theta_{n}(\mathcal{C}) \subset \mathscr{L}_{n}(\xi)^{-1}\left(\theta_{n}(C)\right)
$$

Because $C$ is a cylinder, there exists some $n_{0} \in \mathbb{N}$ and some constructible subset $C_{n_{0}} \subset \mathscr{L}_{n_{0}}(Y)$ such that $C=\left(\theta_{n_{0}}\right)^{-1}\left(C_{n_{0}}\right)$. Then

$$
\mathcal{C}=\mathscr{L}(\xi)^{-1}\left(\left(\theta_{n_{0}}\right)^{-1}\left(C_{n_{0}}\right)\right)=\left(\theta_{n_{0}}\right)^{-1}\left(\mathscr{L}_{n}(\xi)^{-1}\left(C_{n_{0}}\right)\right)
$$

is a cylinder because $\mathscr{L}_{n}(\xi)^{-1}\left(C_{n_{0}}\right)$ is a constructible subset of $\left|\mathscr{L}_{n}(\mathcal{Y})\right|$. We will finish this proof by showing that for any $n \geq n_{0}$, we have $\theta_{n}(\mathcal{C}) \supset \mathscr{L}_{n}(\xi)^{-1}\left(\theta_{n}(C)\right)$.

Set $n \geq n_{0}$ and $\varphi_{n} \in \mathscr{L}_{n}(\xi)^{-1}\left(\theta_{n}(C)\right)$. Because $\mathcal{Y}$ is smooth, there exists some $\varphi \in|\mathscr{L}(\mathcal{Y})|$ such that $\theta_{n}(\varphi)=\varphi_{n}$. Then $\theta_{n}(\mathscr{L}(\xi)(\varphi))=\mathscr{L}_{n}(\xi)\left(\varphi_{n}\right) \in \theta_{n}(C)$, so $\mathscr{L}(\xi)(\varphi) \in\left(\theta_{n}\right)^{-1}\left(\theta_{n}(C)\right)$. But $\left(\theta_{n}\right)^{-1}\left(\theta_{n}(C)\right)=C$, because $C=\left(\theta_{n}\right)^{-1}\left(\left(\theta_{n_{0}}^{n}\right)^{-1}\left(C_{n_{0}}\right)\right)$ is the preimage of a subset of $\mathscr{L}_{n}(Y)$. Thus $\mathscr{L}(\xi)(\varphi) \in C$, which implies $\varphi \in \mathcal{C}$ and $\varphi_{n} \in \theta_{n}(\mathcal{C})$.

We may now prove the special case of Theorem 7.1(a) where $C$ is a cylinder.

Proposition 7.7. Set $w \in \sigma \cap N$. If $C \subset \operatorname{trop}^{-1}(w) \subset \mathscr{L}(X)$ is a cylinder, then $\mathscr{L}(\pi)^{-1}(C) \subset|\mathscr{L}(\mathcal{X})|$ is a cylinder. Furthermore, there exists some $\Theta_{w} \in \widehat{\mathscr{M}}_{k}$ such that for any cylinder $C \subset \operatorname{trop}^{-1}(w) \subset \mathscr{L}(X)$,

$$
\mu_{\mathcal{X}}\left(\mathscr{L}(\pi)^{-1}(C)\right)=\Theta_{w} \mu_{X}(C) .
$$

Proof. Let $n_{w}, \Theta_{w},\left\{\mathcal{F}_{n}\right\}_{n \geq n_{w}}$ be as in the statement of Theorem 5.1, and let $n_{\operatorname{trop}^{-1}(w)}$ be as in the statement of Lemma 7.5 (with $Y=X$ and $C=\operatorname{trop}^{-1}(w)$ ). We show that if $n \geq \max \left\{n_{w}, n_{\text {trop }^{-1}(w)}\right\}$ and $C_{n} \subset \theta_{n}\left(\operatorname{trop}^{-1}(w)\right)$ is constructible, then

$$
\mathrm{e}\left(\mathscr{L}_{n}(\pi)^{-1}\left(C_{n}\right)\right)=\Theta_{w} \mathrm{e}\left(C_{n}\right)
$$

Let $n \geq \max \left\{n_{w}, n_{\text {trop }^{-1}(w)}\right\}$, let $k^{\prime}$ be a field extension of $k$ and set $\varphi_{n} \in \mathscr{L}_{n}(X)\left(k^{\prime}\right)$ with image in $\theta_{n}\left(\operatorname{trop}^{-1}(w)\right)$. Then by our choice of $n_{\text {trop }^{-1}(w)}$, there exists some $\varphi \in \mathscr{L}(X)\left(k^{\prime}\right)$ such that trop $(\varphi)=w$ and $\theta_{n}(\varphi)=\varphi_{n}$. Then by our choice of $n_{w}$ and $\mathcal{F}_{n}$,

$$
\mathscr{L}_{n}(\pi)^{-1}\left(\varphi_{n}\right)_{\mathrm{red}} \cong \mathcal{F}_{n} \otimes_{k} k^{\prime} .
$$

Therefore Proposition 2.5, Remark 2.7, and Proposition 2.8 imply that for any constructible subset $C_{n} \subset \theta_{n}\left(\operatorname{trop}^{-1}(w)\right)$,

$$
\mathrm{e}\left(\mathscr{L}_{n}(\pi)^{-1}\left(C_{n}\right)\right)=\mathrm{e}\left(\mathcal{F}_{n}\right) \mathrm{e}\left(C_{n}\right)=\Theta_{w} \mathrm{e}\left(C_{n}\right)
$$

where the second equality holds by our choice of $n_{w}, \Theta_{w}, \mathcal{F}_{n}$.

Now let $C \subset \operatorname{trop}^{-1}(w)$ be a cylinder, and let $\mathcal{C}=\mathscr{L}(\pi)^{-1}(C) \subset|\mathscr{L}(\mathcal{X})|$. Then $\mathcal{C}$ is a cylinder by Lemma 7.6. Let $n_{0}$ be as in the statement of Lemma 7.6 (with $\xi=\pi$ ). Then for any $n \geq \max \left\{n_{w}, n_{\text {trop }^{-1}(w)}, n_{0}\right\}$,

$$
\mathrm{e}\left(\theta_{n}(\mathcal{C})\right)=\mathrm{e}\left(\mathscr{L}_{n}(\pi)^{-1}\left(\theta_{n}(C)\right)\right)=\Theta_{w} \mathrm{e}\left(\theta_{n}(C)\right)
$$


where the first equality holds by our choice of $n_{0}$. Therefore

$$
\begin{aligned}
\mu_{\mathcal{X}}(\mathcal{C}) & =\lim _{n \rightarrow \infty} \mathrm{e}\left(\theta_{n}(\mathcal{C})\right) \mathbb{L}^{-(n+1) \operatorname{dim} \mathcal{X}} \\
& =\Theta_{w} \lim _{n \rightarrow \infty} \mathrm{e}\left(\theta_{n}(C)\right) \mathbb{L}^{-(n+1) \operatorname{dim} X} \\
& =\Theta_{w} \mu_{X}(C) .
\end{aligned}
$$

Now we may complete the proof of Theorem 7.1(a) in general.

Proof of Theorem 7.1(a). Set $w \in \sigma \cap N$, let $\Theta_{w}$ be as in the statement of Proposition 7.7 and let $C \subset \operatorname{trop}^{-1}(w)$ be a measurable subset of $\mathscr{L}(X)$.

For any $\varepsilon \in \mathbb{R}_{>0}$ and any cylindrical $\varepsilon$-approximation $\left(C^{(0)},\left(C^{(i)}\right)_{i \in I}\right)$ of $C$, Proposition $7.7 \mathrm{im}$ plies that $\left(\mathscr{L}(\pi)^{-1}\left(C^{(0)}\right),\left(\mathscr{L}(\pi)^{-1}\left(C^{(i)}\right)\right)_{i \in I}\right)$ is a cylindrical $\varepsilon\left\|\Theta_{w}\right\|$-approximation of $\mathscr{L}(\pi)^{-1}(C)$. Therefore $\mathscr{L}(\pi)^{-1}(C)$ is measurable, and by another application of Proposition 7.7,

$$
\mu_{\mathcal{X}}\left(\mathscr{L}(\pi)^{-1}(C)\right)=\Theta_{w} \mu_{X}(C)
$$

\subsection{Quotient by an algebraic torus}

In order to apply Theorem 3.9 to prove Theorem 7.1(b), we must rewrite our fantastack as the quotient by a torus. The following result provides an explicit way to do so:

Proposition 7.8. Define $\widehat{N}:=\widetilde{N} \oplus N$ and let $\widehat{\sigma}=\widetilde{\sigma} \times\{0\}$. Letting $\widehat{X}$ be the toric variety associated to $\widehat{\sigma}$, there is a $\widetilde{T}$-action on $\widehat{X}$ and an isomorphism $[\widehat{X} / \widetilde{T}] \stackrel{\sim}{\rightarrow} \mathcal{X}$ such that $\widehat{X} \rightarrow[\widehat{X} / \widetilde{T}] \stackrel{\sim}{\rightarrow} \mathcal{X} \stackrel{\pi}{\rightarrow} X$ is the toric morphism induced by $v \oplus \mathrm{id}: \widehat{N} \rightarrow N$.

Proof. For ease of notation, let $\widehat{v}=v \oplus$ id and consider the stacky fan $(\widehat{\sigma}, \widehat{v})$. One computes that $\operatorname{cok}\left(\widehat{v}^{*}\right)=\widetilde{M}$ and hence $G_{\widehat{v}}=\widetilde{T}$. As a result, $\mathcal{X}_{\widehat{\sigma}, \widehat{v}}=[\widehat{X} / \widetilde{T}]$ for an appropriate $\widetilde{T}$-action on $\widehat{X}$.

Next note that $\mathcal{X}$ is, by definition, the toric stack $\mathcal{X}_{\widetilde{\sigma}, v}$, and consider the following commutative diagram, where the vertical maps are stacky fans and the horizontal maps are morphisms between the stacky fans:

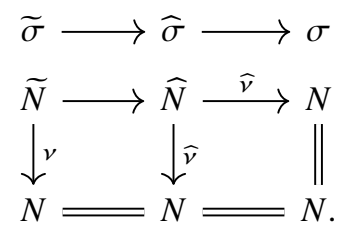

This induces morphisms $\mathcal{X} \rightarrow[\widehat{X} / \widetilde{T}] \rightarrow X$ of toric stacks, and the composite is the morphism $\pi$. By [GS15a, Lemma B.17], the former map is an isomorphism of toric stacks; this is the inverse of our desired isomorphism $[\widehat{X} / \widetilde{T}] \stackrel{\sim}{\rightarrow} \mathcal{X}$. Lastly, we see that the toric morphism $\widehat{X} \rightarrow[\widehat{X} / \widetilde{T}] \rightarrow X$ is induced by the rightmost map in the top row of the diagram, namely $\widehat{v}: \widehat{N} \rightarrow N$.

\subsection{Canonical stacks and preimages of cocharacters}

We end this section by proving Theorem 7.1(b).

For the remainder of this section, let $r=\operatorname{rk} \widetilde{N}$, let $v_{1}, \ldots, v_{r}$ be the first lattice points of the rays of $\sigma$, let $e_{1}, \ldots, e_{r}$ be the generators of $\widetilde{N}$ indexed by the rays $\mathbb{R}_{\geq 0} v_{1}, \ldots, \mathbb{R}_{\geq 0} v_{r}$, so

$$
\beta\left(e_{i}\right)=v_{i}
$$


for all $i \in\{1, \ldots, r\}$, and let $f_{1}, \ldots, f_{r}$ be the basis of $\widetilde{M}$ dual to $e_{1}, \ldots, e_{r}$. Thus $f_{1}, \ldots, f_{r}$ are the minimal generators of the monoid $F$.

Lemma 7.9. We have the equality

$$
m\left(f_{1}+\cdots+f_{r}\right)=q .
$$

Proof. Because the inclusion $P \hookrightarrow F$ is dual to $\beta$,

$$
\left\langle e_{i}, q\right\rangle=m
$$

for all $i \in\{1, \ldots, r\}$. Then

$$
m\left(f_{1}+\cdots+f_{r}\right)=\left\langle e_{1}, q\right\rangle f_{1}+\cdots+\left\langle e_{r}, q\right\rangle f_{r}=q .
$$

For the remainder of this section, let $\widehat{N}, \widehat{\sigma}$ and $\widehat{X}$ be as in Proposition 7.8. Let $\rho: \widehat{X} \rightarrow \mathcal{X}$ be the composition $\widehat{X} \rightarrow[\widehat{X} / \widetilde{T}] \stackrel{\sim}{\rightarrow} \mathcal{X}$, where the $\widetilde{T}$-action on $\widehat{X}$ and the isomorphism $[\widehat{X} / \widetilde{T}] \stackrel{\sim}{\rightarrow} \mathcal{X}$ are as in the statement of Proposition 7.8; let $D_{1}, \ldots, D_{r}$ be the divisors of $\widehat{X}$ indexed by $e_{1} \oplus 0, \ldots, e_{r} \oplus 0 \in \widetilde{N} \oplus N=$ $\widehat{N}$, respectively; and let $\mathcal{O}\left(-D_{1}\right), \ldots, \mathcal{O}\left(-D_{r}\right)$ be the ideal sheaves on $\widehat{X}$ of $D_{1}, \ldots, D_{r}$, respectively. Note that for all $i \in\{1, \ldots, r\}$, the ideal $\mathcal{O}\left(-D_{i}\right)$ is generated by the monomial in $k[F \oplus M]$ indexed by $f_{i} \oplus 0$.

Proposition 7.10. Set $w \in \sigma \cap N$. Then

$$
\mathscr{L}(\rho)^{-1}\left(\mathscr{L}(\pi)^{-1}\left(\operatorname{trop}^{-1}(w)\right)\right)=\bigcup_{\widetilde{w} \in \beta^{-1}(w)}\left(\bigcap_{i=1}^{r} \operatorname{ord}_{\mathcal{O}\left(-D_{i}\right)}^{-1}\left(\left\langle\widetilde{w}, f_{i}\right\rangle\right)\right) .
$$

Proof. By Remark 4.6 and Lemma 4.7(2), it is enough to show that for any $\widehat{f} \in \widehat{\sigma}^{\vee} \cap \widehat{N}^{*}$, there exists $\widehat{f}^{\prime} \in \widehat{\sigma}^{\vee} \cap \widehat{N}^{*}$ such that $\widehat{f}+\widehat{f}^{\prime}$ is in the image of $\sigma^{\vee} \cap M$. By the definition of $\widehat{v}$, the map

$$
P=\sigma^{\vee} \cap M \rightarrow \widehat{\sigma}^{\vee} \cap \widehat{N}^{*}=\left(\widetilde{\sigma}^{\vee} \cap \widetilde{M}\right) \oplus 0=F \oplus 0
$$

is precisely $p \mapsto(p, 0)$. The result then follows from Proposition 2.14.

We may now complete the proof of Theorem 7.1(b).

Proof of Theorem 7.1(b). Set $w \in \sigma \cap N$, and set

$$
\widehat{C}=\mathscr{L}(\rho)^{-1}\left(\mathscr{L}(\pi)^{-1}\left(\operatorname{trop}^{-1}(w)\right)\right) \subset \mathscr{L}(\widehat{X}) .
$$

Then

$$
\begin{aligned}
& \mu_{\widehat{X}}(\widehat{C})=\mu_{\widehat{X}}\left(\bigcup_{\widetilde{w} \in \beta^{-1}(w)}\left(\bigcap_{i=1}^{r} \operatorname{ord}_{\mathcal{O}\left(-D_{i}\right)}^{-1}\left(\left\langle\widetilde{w}, f_{i}\right\rangle\right)\right)\right) \\
& =\sum_{\widetilde{w} \in \beta^{-1}(w)} \mu_{\widehat{X}}\left(\bigcap_{i=1}^{r} \operatorname{ord}_{\mathcal{O}\left(-D_{i}\right)}^{-1}\left(\left\langle\widetilde{w}, f_{i}\right\rangle\right)\right) \\
& =\sum_{\widetilde{w} \in \beta^{-1}(w)}(\mathbb{L}-1)^{r+d} \mathbb{L}^{-(r+d)-\sum_{i=1}^{r}\left\langle\widetilde{w}, f_{i}\right\rangle} \\
& =\sum_{\widetilde{w} \in \beta^{-1}(w)}(\mathbb{L}-1)^{r+d} \mathbb{L}^{-(r+d)-\langle w, q\rangle / m} \\
& =\left(\# \beta^{-1}(w)\right)(\mathbb{L}-1)^{r+d} \mathbb{L}^{-(r+d)-\langle w, q\rangle / m},
\end{aligned}
$$


where the first equality is by Proposition 7.10, the second is by Proposition 2.15 and the fact that the union in the first line is disjoint, the third equality is by [CLNS18, Chapter 7, Lemma 3.3.3] and the definition of $\widehat{X}$ and $D_{1}, \ldots, D_{r}$ and the fourth equality is by Lemma 7.9.

The set $\mathscr{L}(\pi)^{-1}\left(\operatorname{trop}^{-1}(w)\right) \subset|\mathscr{L}(\mathcal{X})|$ is a cylinder, by Remark 4.6 and Proposition 7.7. Then by Theorem 3.9,

$$
\begin{aligned}
\mu_{\mathcal{X}}\left(\mathscr{L}(\pi)^{-1}\left(\operatorname{trop}^{-1}(w)\right)\right) & =\mu_{\widehat{X}}(\widehat{C}) \mathrm{e}(\widetilde{T})^{-1} \mathbb{L}^{\operatorname{dim} \widetilde{T}} \\
& =\left(\# \beta^{-1}(w)\right)(\mathbb{L}-1)^{d} \mathbb{L}^{-d-\langle w, q\rangle / m}
\end{aligned}
$$

\section{Stringy invariants and toric Artin stacks: Proof of Theorem 1.7}

We complete the proof of Theorem 1.7 in this section. Set $d \in \mathbb{N}$, let $N \cong \mathbb{Z}^{d}$ be a lattice and let $T=\operatorname{Spec}\left(k\left[N^{*}\right]\right)$ be the algebraic torus with cocharacter lattice $N$. We recall the following lemma, whose proof is standard:

Lemma 8.1. Let $\sigma$ be a pointed rational cone in $N_{\mathbb{R}}$, and assume that the affine $T$-toric variety associated to it is $\mathbb{Q}$-Gorenstein. Then there exists a d-dimensional pointed rational cone $\bar{\sigma}$ in $N_{\mathbb{R}}$ such that $\sigma$ is a face of $\bar{\sigma}$ and the T-toric variety associeted to $\bar{\sigma}$ is $\mathbb{Q}$-Gorenstein.

Remark 8.2. By Remark 1.8, Proposition 2.18, Proposition 3.27, Proposition 3.31, Proposition 3.32 and Lemma 8.1, to prove Theorem 1.7 it is sufficient to prove the special case where $\mathcal{X}$ is the canonical stack over an affine $T$-toric variety defined by a $d$-dimensional cone in $N_{\mathbb{R}}$.

Set $\sigma$ be a $d$-dimensional pointed rational cone in $N_{\mathbb{R}}$, let $X$ be the affine $T$-toric variety associated to $\sigma$, let $\mathcal{X}$ be the canonical stack over $X$, let $\pi: \mathcal{X} \rightarrow X$ be the canonical map and assume that $X$ is Q-Gorenstein. We will use the notation listed in Notation 2.19.

Proposition 8.3. Set $W \subset \sigma \cap N$. Then

$$
\bigcup_{w \in W} \operatorname{trop}^{-1}(w)
$$

is a measurable subset of $\mathscr{L}(X)$.

Proof. We have that $\mathscr{L}(X \backslash T)$ is a measurable subset of $\mathscr{L}(X)$, because $X \backslash T$ is a closed subscheme of $X$. For each $w \in \sigma \cap N$, the set $\operatorname{trop}^{-1}(w)$ is a measurable subset of $\mathscr{L}(X)$ by Remark 4.6. Also,

$$
\mathscr{L}(X \backslash T) \cup \bigcup_{w \in \sigma \cap N} \operatorname{trop}^{-1}(w)=\mathscr{L}(X)
$$

is measurable and disjoint, so for any $\varepsilon \in \mathbb{R}_{>0}$, there are only finitely many $w \in \sigma \cap N$ such that $\left\|\mu_{X}\left(\operatorname{trop}^{-1}(w)\right)\right\| \geq \varepsilon$. Thus $\bigcup_{w \in W} \operatorname{trop}^{-1}(w)$ is a measurable subset of $\mathscr{L}(X)$.

Proposition 8.4. The function $\operatorname{sep}_{\pi}: \mathscr{L}(X) \rightarrow \mathbb{N}$ has measurable fibres.

Proof. Theorem 4.9 implies that for any $\varphi \in \mathscr{L}(X)$ with $\operatorname{trop}(\varphi) \in \sigma \cap N$,

$$
\operatorname{sep}_{\pi}(\varphi)=\# \beta^{-1}(\operatorname{trop}(\varphi))
$$

Thus, noting that each $\beta^{-1}(w)$ is finite, we have that for any $n \in \mathbb{N}$,

$$
\operatorname{sep}_{\pi}^{-1}(n)=\left(\operatorname{sep}_{\pi}^{-1}(n) \cap \mathscr{L}(X \backslash T)\right) \cup \bigcup_{\substack{w \in \sigma \cap N \\ \# \beta^{-1}(w)=n}} \operatorname{trop}^{-1}(w)
$$


is measurable by Proposition 8.3 and the fact that $\mu_{X}(\mathscr{L}(X \backslash T))=0$, which implies that any subset of $\mathscr{L}(X \backslash T)$ is a measurable subset of $\mathscr{L}(X)$.

For the remainder of this section, we will use the fact that by Proposition 8.4, the integral $\int_{C} \operatorname{sep}{ }_{\pi} \mathrm{d} \mu_{X}^{\text {Gor }}$ is well defined for any measurable subset $C \subset \mathscr{L}(X)$.

We end this section with the next proposition, which along with Remark 8.2 and Proposition 8.4 implies Theorem 1.7.

Proposition 8.5. Let $C$ be a measurable subset of $\mathscr{L}(X)$. Then $\mathscr{L}(\pi)^{-1}(C)$ is a measurable subset of $|\mathscr{L}(\mathcal{X})|$ and

$$
\mu_{\mathcal{X}}\left(\mathscr{L}(\pi)^{-1}(C)\right)=\int_{C} \operatorname{sep}_{\pi} \mathrm{d} \mu_{X}^{\text {Gor }}
$$

Proof. By Proposition 2.3, Proposition 7.3 and the fact that

$$
\mu_{X}\left(\mathscr{L}(X) \backslash \bigcup_{w \in \sigma \cap N} \operatorname{trop}^{-1}(w)\right)=\mu_{X}(\mathscr{L}(X \backslash T))=0,
$$

it is enough to prove the statement for each $\operatorname{trop}^{-1}(w) \cap C$. In other words, we may fix $w$ and assume $C \subset \operatorname{trop}^{-1}(w)$.

We first note that $\mathscr{L}(\pi)^{-1}(C) \subset|\mathscr{L}(\mathcal{X})|$ is measurable, by Theorem 7.1(a). Let $m \in \mathbb{Z}_{>0}$ and $q \in P$ be such that $\langle v, q\rangle=m$ for any first lattice point $v$ of a ray of $\sigma$. Let $j_{w}$ be as in the statement of Theorem 6.1(a), and let $\Theta_{w}$ be as in the statement of Theorem 7.1(a). By Theorem 6.1(b) and our choice of $j_{w}$,

$$
\left(\mathbb{L}^{1 / m}\right)^{j_{w}} \mu_{X}\left(\operatorname{trop}^{-1}(w)\right)=\mu_{X}^{\mathrm{Gor}}\left(\operatorname{trop}^{-1}(w)\right)=\mathbb{L}^{-d}(\mathbb{L}-1)^{d}\left(\mathbb{L}^{1 / m}\right)^{-\langle w, q\rangle} .
$$

In particular, $\mu_{X}\left(\operatorname{trop}^{-1}(w)\right)$ is a unit in $\widehat{\mathscr{M}_{k}}\left[\mathbb{L}^{1 / m}\right]$. Then by the foregoing equality, Theorem 7.1(b) and our choice of $\Theta_{w}$,

$$
\begin{aligned}
\left(\# \beta^{-1}(w)\right)\left(\mathbb{L}^{1 / m}\right)^{j_{w}} \mu_{X}\left(\operatorname{trop}^{-1}(w)\right) & =\left(\# \beta^{-1}(w)\right) \mathbb{L}^{-d}(\mathbb{L}-1)^{d}\left(\mathbb{L}^{1 / m}\right)^{-\langle w, q\rangle} \\
& =\mu_{\mathcal{X}}\left(\mathscr{L}(\pi)^{-1}\left(\operatorname{trop}^{-1}(w)\right)\right) \\
& =\Theta_{w} \mu_{X}\left(\operatorname{trop}^{-1}(w)\right),
\end{aligned}
$$

so

$$
\Theta_{w}=\left(\# \beta^{-1}(w)\right)\left(\mathbb{L}^{1 / m}\right)^{j_{w}}
$$

Therefore, by our choice of $\Theta_{w}$ and $j_{w}$,

$$
\begin{aligned}
\mu_{\mathcal{X}}\left(\mathscr{L}(\pi)^{-1}(C)\right) & =\Theta_{w} \mu_{X}(C) \\
& =\left(\# \beta^{-1}(w)\right)\left(\mathbb{L}^{1 / m}\right)^{j_{w}} \mu_{X}(C) \\
& =\left(\# \beta^{-1}(w)\right) \mu_{X}^{\mathrm{Gor}}(C) \\
& =\int_{C} \operatorname{sep}_{\pi} \mathrm{d} \mu_{X}^{\mathrm{Gor}}
\end{aligned}
$$

where the last equality is by Theorem 4.9. 


\section{Fantastacks with special stabilisers: Proof of Theorem 1.11}

The goal of this section is to prove Theorem 9.1, which characterises when a fantastack has only special stabilisers, and then to use this characterisation to prove Theorem 1.11. For simplicity, we state the criterion Theorem 9.1 only in the case where the good moduli space is affine (and defined by a fulldimensional cone).

Throughout this section, set $d \in \mathbb{N}$, let $N \cong \mathbb{Z}^{d}$ be a lattice and let $T=\operatorname{Spec}\left(k\left[N^{*}\right]\right)$ be the algebraic torus with cocharacter lattice $N$.

Theorem 9.1. Let $\mathcal{X}=\mathcal{F}_{\sigma, v}$ be a fantastack with dense torus $T$ and keep the notation listed in Definition 2.12. Then the following are equivalent:

(i) The stabilisers of $\mathcal{X}$ are all special groups.

(ii) For all $I \subset\{1, \ldots, r\}$, the set $\left\{v\left(e_{i}\right) \mid i \in I\right\}$ is linearly independent if and only if it can be extended to a basis for $N$.

(iii) For some $n \in \mathbb{N}$, we have $\mathcal{X} \cong\left[\mathbb{A}_{k}^{r} / \mathbb{G}_{m}^{n}\right]$, where $\mathbb{G}_{m}^{n}$ acts on $\mathbb{A}_{k}^{r}$ with weights $w_{1}, \ldots, w_{r} \in \mathbb{Z}^{n}$ such that for all $I \subset\{1, \ldots, r\}$, the set $\left\{w_{i} \mid i \in I\right\}$ is linearly independent if and only if it can be extended to a basis for $\mathbb{Z}^{n}$.

The following is an immediate consequence of Theorem 9.1, noting that taking a canonical stack is compatible with taking products of toric varieties:

Corollary 9.2. Let $\sigma$ be a pointed rational cone in $N_{\mathbb{R}}$, and let $\mathcal{X}$ be the canonical stack over $X_{\sigma}$. If $v_{1}, \ldots, v_{r} \in N$ are the first lattice points of the rays of $\sigma$, then the following are equivalent:

(i) The stabilisers of $\mathcal{X}$ are all special groups.

(ii) For all $I \subset\{1, \ldots, r\}$, the set $\left\{v_{i} \mid i \in I\right\}$ is linearly independent if and only if it can be extended to a basis for $N$.

Before proving Theorem 9.1, we use Corollary 9.2 to prove Theorem 1.11.

Proof of Theorem 1.11. Let $X$ be a toric variety over $k$, let $\pi: \mathcal{X} \rightarrow X$ be its canonical stack (see Remark 2.21) and assume that the stabilisers of $\mathcal{X}$ are all special groups. By Proposition 2.18 and the definition of sep $_{\pi}$, we may assume that $X$ is the affine $T$-toric variety defined by a pointed rational cone $\sigma$ in $N_{\mathbb{R}}$. It is easy to check, for example by using Corollary 9.2, that because the stabilisers of $\mathcal{X}$ are all special groups, the cone $\sigma$ is a face of a $d$-dimensional pointed rational cone in $N_{\mathbb{R}}$ whose associated toric variety has a canonical stack with only special stabilisers. Therefore we may assume that $\sigma$ is $d$-dimensional and use the notation listed in Notation 2.19. Then by Proposition 2.15, Theorem 4.9 and the fact that

$$
\mu_{X}\left(\mathscr{L}(X) \backslash \bigcup_{w \in \sigma \cap N} \operatorname{trop}^{-1}(w)\right)=\mu_{X}(\mathscr{L}(X \backslash T))=0
$$

it is sufficient to show that $\beta$ is surjective.

Set $w \in \sigma \cap N$. We will use Corollary 9.2 to show that $w$ is in the image of $\beta$. Let $\Sigma$ be a simplicial subdivision of $\sigma$ whose rays are all rays of $\sigma$, and let $\sigma_{w} \in \Sigma$ be a cone containing $w$. By Corollary 9.2, the cone $\sigma_{w}$ is unimodular, so $w$ is a positive integer combination of first lattice points of rays of $\sigma$. Therefore $w$ is in the image of $\beta$ by the definition of $\beta$, and we are done.

The remainder of this paper will be used to prove Theorem 9.1 .

\subsection{A combinatorial criterion for special stabilisers}

We start with some preliminary results, the first of which is a standard fact. 
Lemma 9.3. If $A$ and $B$ are finite-rank lattices and

$$
0 \rightarrow A \stackrel{f}{\rightarrow} B \rightarrow C \rightarrow 0
$$

is a short exact sequence, then $\operatorname{cok}\left(f^{*}\right)$ is finite. Moreover, $C$ is torsion-free if and only if $f^{*}$ is surjective.

Proof. Letting $C_{\text {tor }}$ be the torsion part of $C$, applying $\operatorname{Hom}(-, \mathbb{Z})$ to the short exact sequence

$$
0 \rightarrow C_{\text {tor }} \rightarrow C \rightarrow \bar{C} \rightarrow 0
$$

we see that $\operatorname{Ext}^{1}(C, \mathbb{Z}) \cong \operatorname{Ext}^{1}\left(C_{\text {tor }}, \mathbb{Z}\right)$, which is finite. Then from the exact sequence

$$
0 \rightarrow C^{*} \rightarrow B^{*} \stackrel{f^{*}}{\rightarrow} A^{*} \rightarrow \operatorname{Ext}^{1}(C, \mathbb{Z}) \rightarrow 0
$$

we see that $\operatorname{cok}\left(f^{*}\right)$ is finite and that $f^{*}$ is surjective if and only if $\operatorname{Ext}^{1}(C, \mathbb{Z})=0$ if and only if $C$ is a lattice.

Lemma 9.4. Let $A$ be a lattice and suppose $v_{1}, \ldots, v_{r} \in A$ span $A_{\mathbb{Q}}$. Then the following conditions are equivalent:

1. $A / \sum_{i \in S} \mathbb{Z} v_{i}$ is torsion-free for all $S \subseteq\{1, \ldots, r\}$.

2. For every $S \subseteq\{1, \ldots, r\}$, if $\left\{v_{i} \mid i \in S\right\}$ is a $\mathbb{Q}$-basis for $A_{\mathbb{Q}}$, then it is a $\mathbb{Z}$-basis for $A$.

Proof. To ease notation, define $L_{S}:=\sum_{i \in S} \mathbb{Z} v_{i}$ and $L_{S}^{\text {sat }}:=A \cap\left(L_{S}\right)_{\mathbb{Q}}$ for all $S \subseteq\{1, \ldots, r\}$. Note that $A / L_{S}^{\text {sat }}$ is torsion-free, so $L_{S}^{\text {sat }}$ is a direct summand of $A$. It follows that $A / L_{S}$ is torsion-free if and only if $L_{S}^{\text {sat }} / L_{S}$ is torsion-free, and since $L_{S}^{\text {sat }} / L_{S}$ is finite, we see that condition (1) is equivalent to $L_{S}=L_{S}^{\text {sat }}$.

Now suppose that condition (1) holds and set $S \subseteq\{1, \ldots, r\}$ such that $\left\{v_{i} \mid i \in S\right\}$ is a $\mathbb{Q}$-basis for $A_{\mathbb{Q}}$. Then $L_{S}=L_{S}^{\text {sat }}=A$, so $\left\{v_{i} \mid i \in S\right\}$ is a $\mathbb{Z}$-basis for $A$.

Conversely, suppose condition (2) holds, and let $S \subseteq\{1, \ldots, r\}$ be any subset. Choose $S^{\prime} \subseteq S$ such that $\left\{v_{i} \mid i \in S^{\prime}\right\}$ form a $\mathbb{Q}$-basis for $\left(L_{S}\right)_{\mathbb{Q}}$. Since the $\mathbb{Q}$-span of $v_{1}, \ldots, v_{r}$ is $A_{\mathbb{Q}}$, we can choose $S^{\prime \prime} \subseteq\{1, \ldots, r\} \backslash S$ such that $\left\{v_{i} \mid i \in S^{\prime} \cup S^{\prime \prime}\right\}$ form a $\mathbb{Q}$-basis for $A_{\mathbb{Q}}$. It follows that $\left\{v_{i} \mid i \in S^{\prime} \cup S^{\prime \prime}\right\}$ is a $\mathbb{Z}$-basis for $A$, and hence $A \cong L_{S^{\prime}} \oplus L_{S^{\prime \prime}}$.

To show that condition (1) holds - that is, $A / L_{S}$ is torsion-free - it thus suffices to show $L_{S^{\prime}}=L_{S}$. To see why this equality holds, set $j \in S$ and write $v_{j}=\sum_{i \in S^{\prime}} a_{i}^{\prime} v_{i}+\sum_{i \in S^{\prime \prime}} a_{i}^{\prime \prime} v_{i}$, with $a_{i}^{\prime}, a_{i}^{\prime \prime} \in \mathbb{Z}$. On the other hand, by the definition of $S^{\prime}$, we can write $v_{j}=\sum_{i \in S^{\prime}} b_{i}^{\prime} v_{i}$, with $b_{i}^{\prime} \in \mathbb{Q}$. Equating our two expressions and using the fact that $\left\{v_{i} \mid i \in S^{\prime} \cup S^{\prime \prime}\right\}$ is a $\mathbb{Q}$-basis for $A_{\mathbb{Q}}$, we see that $a_{i}^{\prime \prime}=0$ for all $i \in S^{\prime \prime}$. So $v_{j} \in L_{S^{\prime}}$.

Lemma 9.5. Let $\mathcal{X}=\left[\mathbb{A}_{k}^{r} / \mathbb{G}_{m}^{n}\right]$, where $\mathbb{G}_{m}^{n}$ acts with weights $w_{1}, \ldots, w_{r} \in \mathbb{Z}^{n}$ which span $\mathbb{Q}^{n} .^{2}$ Then $\mathcal{X}$ has special stabilisers if and only if for every $S \subseteq\{1, \ldots, r\}$, if $\left\{w_{i} \mid i \in S\right\}$ is a $\mathbb{Q}$-basis for $\mathbb{Q}^{n}$, then it is a $\mathbb{Z}$-basis for $\mathbb{Z}^{n}$.

Proof. Since the stabilisers of $\mathcal{X}$ are subgroups of $\mathbb{G}_{m}^{n}$, they are special if and only if they are connected. Let $w_{i}=\left(a_{i 1}, \ldots, a_{i n}\right) \in \mathbb{Z}^{n}$. Given a point $x=\left(x_{1}, \ldots, x_{r}\right) \in \mathbb{A}_{k}^{r}$, let $S_{x}=\left\{i \mid x_{i} \neq 0\right\}$. Then the stabiliser $G_{x}$ of $x$ is the set of $\left(\lambda_{1}, \ldots, \lambda_{n}\right) \in \mathbb{G}_{m}^{n}$ such that $\prod_{j=1}^{n} \lambda_{j}^{a_{i j}}=1$ for all $i \in S_{x}$. In other words, we have a short exact sequence

$$
1 \rightarrow G_{x} \rightarrow \mathbb{G}_{m}^{n} \stackrel{\varphi}{\rightarrow} \mathbb{G}_{m}^{S_{x}} \rightarrow 1
$$

where for $i \in S_{x}$, the $i$ th coordinate of $\varphi\left(\lambda_{1}, \ldots, \lambda_{n}\right)$ is given by $\prod_{j=1}^{n} \lambda_{j}^{a_{i j}}$. Taking Cartier duals $D(-):=\operatorname{Hom}\left(-, \mathbb{G}_{m}\right)$, we see that $D\left(G_{x}\right)$ is the cokernel of the map $\mathbb{Z}^{S_{x}} \rightarrow \mathbb{Z}^{n}$ sending the $i$ th standard basis vector to $w_{i}$. By Cartier duality, $G_{x}$ is connected if and only if $D\left(G_{x}\right)$ is torsion-free.

${ }^{2}$ Note that we do not assume $\mathcal{X}$ is a fantastack here. 
We have thus shown that $\mathcal{X}$ has connected stabilisers if and only if $\mathbb{Z}^{n} / \sum_{i \in S} \mathbb{Z} w_{i}$ is torsion-free for all subsets $S \subseteq\{1, \ldots, r\}$. Lemma 9.4 then finishes the proof.

We now turn to Theorem 9.1.

Proof of Theorem 9.1. The map $v: \mathbb{Z}^{r} \rightarrow N$ has finite cokernel, or equivalently, $v^{*}$ is injective. So we have a short exact sequence

$$
0 \longrightarrow M \stackrel{v^{*}}{\longrightarrow}\left(\mathbb{Z}^{r}\right)^{*} \stackrel{\alpha}{\longrightarrow} A \longrightarrow 0
$$

By the construction of fantastacks, we have $\mathcal{X}=\left[\mathbb{A}_{k}^{r} / G\right]$, where $G$ is the Cartier dual of $A$. Note that $G$ is the stabiliser of the origin, and it is connected if and only if $A$ is torsion-free. By Lemma 9.3, this is equivalent to surjectivity of $v$.

So we may now assume $v$ is surjective, and in light of Lemma 9.5, we need only establish the equivalence of conditions (ii) and (iii). By Lemma 9.4, condition (ii) holds if and only if cok $\left(\left.v\right|_{\mathbb{Z}^{s}}\right)$ is torsion-free for all subsets $S \subseteq\{1, \ldots, r\}$. Letting $e_{i}^{*} \in\left(\mathbb{Z}^{r}\right)^{*}$ denote the dual linear functional, notice that $\alpha\left(e_{i}^{*}\right)$ is the $i$ th weight for the $G$-action on $\mathbb{A}_{k}^{r}$. Given any subset $S \subseteq\{1, \ldots, r\}$, we let $S^{\prime}$ denote the complement of $S$. We have a natural inclusion $\left(\mathbb{Z}^{S^{\prime}}\right)^{*} \subseteq\left(\mathbb{Z}^{r}\right)^{*}$ with cokernel $\left(\mathbb{Z}^{S}\right)^{*}$. Another application of Lemma 9.4 shows that condition (iii) holds if and only if $Q_{S}:=A / \alpha\left(\left(\mathbb{Z}^{S^{\prime}}\right)^{*}\right)$ is torsion-free for all $S$. We show that this latter condition is equivalent to cok $\left(\left.v\right|_{\mathbb{Z} S}\right)$ being torsion-free for all subsets $S \subseteq\{1, \ldots, r\}$.

Consider the diagram

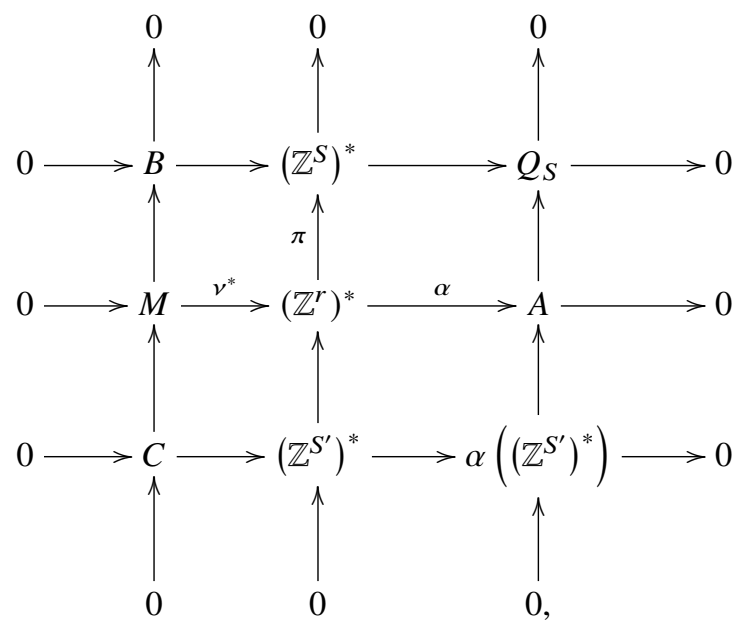

where $B$ is the image of $\pi \circ v^{*}$ and $C=\left(\mathbb{Z}^{S^{\prime}}\right)^{*} \cap \operatorname{ker} \alpha$; in particular, all rows and columns are exact. Note that all $\mathbb{Z}$-modules in this diagram are torsion-free, with the possible exception of $Q_{S}$. Applying $\operatorname{Hom}(-, \mathbb{Z})$, we have the diagram

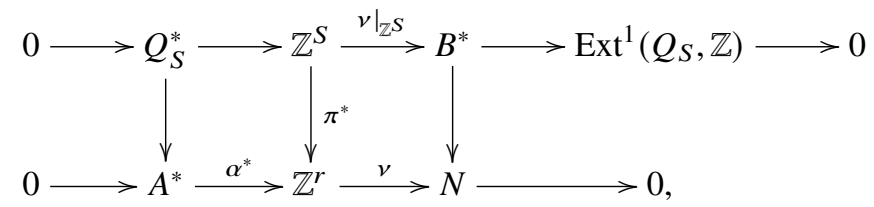


where all rows are exact and all vertical maps are injective. We see then that

$$
\operatorname{cok}\left(\left.v\right|_{\mathbb{Z}^{S}}\right)=\operatorname{Ext}^{1}\left(Q_{S}, \mathbb{Z}\right) .
$$

Letting $Q_{S \text {,tor }} \subseteq Q_{S}$ denote the torsion part, we have $\operatorname{Ext}^{1}\left(Q_{S}, \mathbb{Z}\right)=\operatorname{Ext}^{1}\left(Q_{S}\right.$,tor, $\left.\mathbb{Z}\right)$, which is finite, so $\operatorname{cok}\left(\left.v\right|_{\mathbb{Z}^{S}}\right)$ is torsion-free if and only if $\operatorname{Ext}^{1}\left(Q_{S}, \mathbb{Z}\right)=0$ if and only if $Q_{S}$ is torsion-free.

\section{Quotients by $\mathrm{SL}_{2}$ and cones over Grassmannians}

Until now, we have only considered stacks with abelian stabilisers. In this section, we verify Conjecture 1.1 and Conjecture 1.2 for nontrivial examples where the stabilisers are nonabelian. Our primary running example in this section is a stack $\mathcal{X}=\left[\mathbb{A}^{8} / \mathrm{SL}_{2}\right]$ whose good moduli space is the affine cone over the Grassmannian $\operatorname{Gr}(2,4)$ with respect to the Plücker embedding; we will additionally verify Conjecture 1.2 and answer Question 1.4 for a stack $\mathcal{X}=\left[\mathbb{A}^{2 r} / \mathrm{SL}_{2}\right]$ whose good moduli space is the affine cone over the Grassmannian $\operatorname{Gr}(2, r)$. The cases we consider are particularly interesting because, unlike fantastacks, we show here that $\mu_{X}\left(\operatorname{sep}_{\pi}^{-1}(\infty)\right) \neq 0$.

We begin by setting up some notation. Throughout this section, fix some $r \in \mathbb{Z}_{>1}$ and let $[r]=$ $\{1, \ldots, r\}$. Let $\widetilde{X}=\mathbb{A}^{2 \times r}$, which we think of as $2 \times r$ matrices with coordinates

$$
\left(\begin{array}{lll}
y_{1,1} & \cdots & y_{1, r} \\
y_{2,1} & \cdots & y_{2, r}
\end{array}\right) \text {. }
$$

There is an $\mathrm{SL}_{2}$-action on $\widetilde{X}$ given by left multiplication. The invariant functions are generated by the $2 \times 2$ minors, and so the quotient $X=\widetilde{X} / \mathrm{SL}_{2} \subset \mathbb{A}_{k}^{\left(\left[\begin{array}{c}{[r]} \\ 2\end{array}\right)\right.}$ is the affine cone over the Grassmannian $\operatorname{Gr}(2, r)$ with respect to the Plücker embedding of $\operatorname{Gr}(2, r)$. Letting $\left\{x_{\ell, m} \mid 1 \leq \ell<m \leq r\right\}$ be the coordinates on $\mathbb{A}_{k}^{\left(\begin{array}{c}{[r]} \\ 2\end{array}\right)}$, the quotient map $\widetilde{X} \rightarrow X$ sends each $\left.x_{\ell, m}\right|_{X}$ to the $(\ell, m)$ th minor of the matrix. Set $\mathcal{X}=\left[\widetilde{X} / \mathrm{SL}_{2}\right]$, let $\rho: \widetilde{X} \rightarrow \mathcal{X}$ be the quotient map and let $\pi: \mathcal{X} \rightarrow X$ be the good moduli space map. Note that all stabilisers of $\mathcal{X}$ are special: the zero matrix has stabiliser $\mathrm{SL}_{2}$, a full-rank matrix has trivial stabiliser and a rank 1 matrix has stabiliser $\mathbb{G}_{a}$.

Remark 10.1. When $r \geq 3$, the exceptional locus of $\pi$ has codimension at least 2: it is straightforward to check that if $U \subset X$ is the intersection of $X$ with the complement $\mathbb{G}_{m}^{\left(\begin{array}{c}{[n]} \\ 2\end{array}\right)}$ of the coordinate axes of $\mathbb{A}_{k}^{\left(\begin{array}{c}n] \\ 2\end{array}\right)}$, then $\left.\pi\right|_{\pi^{-1}(U)}: \pi^{-1}(U) \rightarrow U$ is an isomorphism; and when $r \geq 3$, the complement of $\pi^{-1}(U)$ in $\mathcal{X}$ has codimension at least 2 .

The next two propositions verify Conjecture 1.2 and answer Question 1.4.

Proposition 10.2. For $r \geq 2$, Conjecture 1.2 holds for $\pi: \mathcal{X} \rightarrow X$.

Proposition 10.3. For $r \geq 2$, we have $\mu_{X}\left(\operatorname{sep}_{\pi}^{-1}(\infty)\right) \neq 0$, answering Question 1.4 for $\pi: \mathcal{X} \rightarrow X$.

For any w $\in(\mathbb{N} \cup\{\infty\})^{\left(\begin{array}{c}{[r]} \\ 2\end{array}\right)}$, let $C^{(\mathbf{w})} \subset \mathscr{L}(X)$ denote the subset of arcs whose prescribed vanishing orders with respect to the Plücker coordinate are given by $\mathbf{w}$. More precisely,

$$
C^{(\mathbf{w})}=\bigcap_{\ell<m \in[r]} \operatorname{ord}_{x_{\ell, m} \mid X}^{-1}\left(w_{\ell, m}\right) \subset \mathscr{L}(X) .
$$

Note that if $\mathbf{w} \in \mathbb{N}^{\left(\begin{array}{c}r r \\ 2\end{array}\right)}$, then $C^{(\mathbf{w})}$ is a cylinder.

Remark 10.4. If $\mathbf{w} \in\left((\mathbb{N} \cup\{\infty\})^{\left(\begin{array}{c}{[r]} \\ 2\end{array}\right)} \backslash \mathbb{N}\left(\begin{array}{c}{[r]} \\ 2\end{array}\right)\right)$, then $C^{(\mathbf{w})}$ is a measurable set with $\mu_{X}\left(C^{(\mathbf{w})}\right)=0$.

With this notation, we now prove Proposition 10.2 and Proposition 10.3. 
Proof of Proposition 10.2. It suffices to show that away from a set of measure 0 , every $\operatorname{arc} \varphi \in \mathscr{L}(X)\left(k^{\prime}\right)$ lifts to an $\operatorname{arc}$ in $\mathscr{L}(\mathcal{X})\left(k^{\prime}\right)$, where $k^{\prime}$ is an extension field of $k$. By Remark 10.4, we may assume $\varphi \in C^{(\mathbf{w})}$

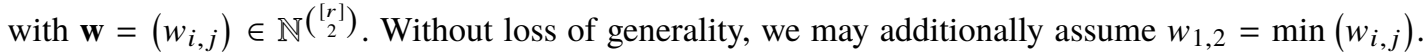
Let the map on coordinate rings induced by $\varphi: \operatorname{Spec}\left(k^{\prime} \llbracket t \rrbracket\right) \rightarrow X$ send $\left.x_{i, j}\right|_{X}$ to $g_{i, j} \in k^{\prime} \llbracket t \rrbracket$. Let $\widetilde{\psi} \in \mathscr{L}(\widetilde{X})$ be the arc given by the matrix

$$
\left(\begin{array}{cccccc}
g_{1,2} & 0 & -g_{2,3} & -g_{2,4} & \cdots & -g_{2, r} \\
0 & 1 & g_{1,3} g_{1,2}^{-1} & g_{1,4} g_{1,2}^{-1} & \cdots & g_{1, r} g_{1,2}^{-1}
\end{array}\right)
$$

Note that all $g_{i, j}$ are nonzero, since $\mathbf{w} \in \mathbb{N}\left(\begin{array}{c}(r] \\ 2\end{array}\right)$, and all entries of the matrix are in $k^{\prime} \llbracket t \rrbracket$, since $w_{1,2}=\min \left(w_{i, j}\right)$. Note further that the $(i, j)$ th minor of the matrix is precisely $g_{i, j}$; this is clear when $i \in\{1,2\}$, so the Plücker relations ensure that it remains true for all $(i, j)$. As a result, $\widetilde{\psi}$ is a lift of $\varphi$, and hence $\psi=\mathscr{L}(\rho)(\widetilde{\psi}) \in \mathscr{L}(\mathcal{X})$ is a lift of $\varphi$.

Proof of Proposition 10.3. As in the proof of Proposition 10.2, it suffices to show that $\mathrm{sep}_{\pi}$ is infinite on $C^{(\mathbf{w})}$ under the assumption that $\mathbf{w}=\left(w_{i, j}\right) \in \mathbb{N}^{\left(\begin{array}{c}r] \\ 2\end{array}\right)}$ and $1 \leq w_{1,2}=\min \left(w_{i, j}\right)$. Again, set $\varphi \in C^{(\mathbf{w})}$ and let $\varphi: \operatorname{Spec}\left(k^{\prime} \llbracket t \rrbracket\right) \rightarrow X$ send $\left.x_{i, j}\right|_{X}$ to $g_{i, j} \in k^{\prime} \llbracket t \rrbracket$. Then for any $h \in k^{\prime} \llbracket t \rrbracket$, we obtain a lift $\widetilde{\psi}_{h} \in \mathscr{L}(\widetilde{X})$ of $\varphi$, where $\widetilde{\psi}_{h}$ is the arc given by the matrix

$$
\left(\begin{array}{cccccc}
g_{1,2} & 0 & -g_{2,3} & -g_{2,4} & \cdots & -g_{2, r} \\
h & 1 & \left(g_{1,3}-h g_{2,3}\right) g_{1,2}^{-1} & \left(g_{1,4}-h g_{2,4}\right) g_{1,2}^{-1} & \cdots & \left(g_{1, r}-h g_{2, r}\right) g_{1,2}^{-1}
\end{array}\right) .
$$

Notice that for any $h, h^{\prime} \in k^{\prime} \llbracket t \rrbracket$ for which $h^{\prime}-h$ is not divisible by $t^{w_{1,2}}$, there is no $A \in \mathrm{SL}_{2}\left(k^{\prime} \llbracket t \rrbracket\right)$ for which $A \widetilde{\psi}_{h}=\widetilde{\psi}_{h^{\prime}}$. Indeed, the unique $A \in \mathrm{SL}_{2}\left(k^{\prime}(t)\right)$ with

$$
A\left(\begin{array}{cc}
g_{1,2} & 0 \\
h & 1
\end{array}\right)=\left(\begin{array}{cc}
g_{1,2} & 0 \\
h^{\prime} & 1
\end{array}\right)
$$

is given by

$$
A=\left(\begin{array}{cc}
1 & 0 \\
\left(h^{\prime}-h\right) g_{1,2}^{-1} & 1
\end{array}\right)
$$

which is not in $\mathrm{SL}_{2}\left(k^{\prime} \llbracket t \rrbracket\right)$, by the assumption on $h^{\prime}-h$. Noting that $w_{1,2} \geq 1$, it follows that $\operatorname{sep}_{\pi}$ is infinite on $C^{(\mathbf{w})}$.

There are two features of the proof of Proposition 10.2 that we wish to highlight. First, for the matrices in expression (3) to define arcs of $\mathscr{L}(\mathcal{X})$, we needed not $w_{1,2}=\min \left(w_{i, j}\right)$ but rather $w_{1,2} \leq$ $w_{1,3}, \ldots, w_{1, r}, w_{2,3}, \ldots, w_{2, r}$. Second, for any $i \leq w_{1,2}$ we have many more lifts of $\varphi$ given by matrices of the form

$$
\left(\begin{array}{cccccc}
g_{1,2} t^{-i} & 0 & -g_{2,3} t^{-i} & -g_{2,4} t^{-i} & \cdots & -g_{2, r} t^{-i} \\
0 & t^{i} & g_{1,3} g_{1,2}^{-1} t^{i} & g_{1,4} g_{1,2}^{-1} t^{i} & \cdots & g_{1, r} g_{1,2}^{-1} t^{i}
\end{array}\right) .
$$

With this as motivation, we introduce the following sets. For any $i, w_{1,2} \in \mathbb{N}$ and $\mathbf{w}_{1}=$ $\left(w_{1,3}, \ldots, w_{1, r}\right), \mathbf{w}_{2}=\left(w_{2,3}, \ldots, w_{2, r}\right) \in \mathbb{N}^{r-2}$ satisfying

$$
2 i \leq w_{1,2} \leq w_{1,3}, \ldots, w_{1, r}, w_{2,3}, \ldots, w_{2, r},
$$


let $Z^{\left(i, w_{1,2}, \mathbf{w}_{1}, \mathbf{w}_{2}\right)}$ be the subset of $\mathscr{L}(\widetilde{X})$ whose $k^{\prime}$-valued points, for any extension $k^{\prime}$ of $k$, are the $2 \times r$ matrices of the form

$$
\left(\begin{array}{cccccc}
f_{1,1} & 0 & f_{1,3} & f_{1,4} & \cdots & f_{1, r} \\
0 & t^{i} & f_{2,3} & f_{2,4} & \cdots & f_{2, r}
\end{array}\right),
$$

where $f_{1,1}, f_{1,3}, \ldots, f_{1, r}, f_{2,3}, \ldots, f_{2, r} \in k^{\prime} \llbracket t \rrbracket$ satisfies

$$
\begin{aligned}
& \operatorname{ord}_{t}\left(f_{1,1}\right)=w_{1,2}-i, \\
& \operatorname{ord}_{t}\left(f_{1, j}\right)=w_{2, j}-i, \\
& \operatorname{ord}_{t}\left(f_{2, j}\right)=w_{1, j}-w_{1,2}+i,
\end{aligned}
$$

for all $j=3, \ldots, r$, for all $j=3, \ldots, r$.

Note that the condition $(\star)$ guarantees that these vanishing orders are nonnegative. For any $i, w_{1,2} \in \mathbb{N}$ and $\mathbf{w}_{1}, \mathbf{w}_{2} \in \mathbb{N}^{r-2}$ satisfying $(\star)$, set

$$
\mathcal{C}^{\left(i, w_{1,2}, \mathbf{w}_{1}, \mathbf{w}_{2}\right)}=\mathscr{L}(\rho)\left(Z^{\left(i, w_{1,2}, \mathbf{w}_{1}, \mathbf{w}_{2}\right)}\right) \subset|\mathscr{L}(\mathcal{X})|
$$

and set

$$
C^{\left(w_{1,2}, \mathbf{w}_{1}, \mathbf{w}_{2}\right)}=\bigcup_{\mathbf{w}} C^{(\mathbf{w})},
$$

where w varies over all elements of $(\mathbb{N} \cup\{\infty\})\left(\begin{array}{c}{\left[\begin{array}{c}r \\ 2\end{array}\right)} \\ \text { w }\end{array}\right)$ whose $(\ell, m)$ th entry is equal to $w_{\ell, m}$ for all $(\ell, m)=(1,2),(1,3), \ldots,(1, r),(2,3), \ldots,(2, r)$.

Last, although (for ease of notation) we have chosen to define all of these sets with $w_{1,2}$ playing a special role, by symmetry of the Plücker coordinates, we obtain many analogous sets as follows. Note that there is a natural $S_{r}$-action on $\mathcal{X}$ given by permuting columns of the matrices in $\widetilde{X}$; by functoriality, this descends to an $S_{r}$-action on the good moduli space $X$. Let

$$
\mathcal{C}_{\sigma}^{\left(i, w_{1,2}, \mathbf{w}_{1}, \mathbf{w}_{2}\right)}=\sigma\left(\mathcal{C}^{\left(i, w_{1,2}, \mathbf{w}_{1}, \mathbf{w}_{2}\right)}\right) \subset|\mathscr{L}(\mathcal{X})| \quad \text { and } \quad C_{\sigma}^{\left(w_{1,2}, \mathbf{w}_{1}, \mathbf{w}_{2}\right)}=\sigma\left(C^{\left(w_{1,2}, \mathbf{w}_{1}, \mathbf{w}_{2}\right)}\right) \subset \mathscr{L}(X) .
$$

Definition 10.5. Let $\mathcal{S}^{\prime}$ be the collection of pairs

$$
\left(\mathcal{C}_{\sigma}^{\left(i, w_{1,2}, \mathbf{w}_{1}, \mathbf{w}_{2}\right)}, C_{\sigma}^{\left(w_{1,2}, \mathbf{w}_{1}, \mathbf{w}_{2}\right)}\right)
$$

with $i, w_{1,2} \in \mathbb{N}$ and $\mathbf{w}_{1}, \mathbf{w}_{2} \in \mathbb{N}^{r-2}$ satisfying $(\star)$, and $\sigma \in S_{r}$. Let $\mathcal{S} \subset \mathcal{S}^{\prime}$ be the subset consisting of pairs where $i>0$.

Remark 10.6. Since the $S_{r}$-actions on $\mathscr{L}(\mathcal{X})$ and $\mathscr{L}(X)$ are measure-preserving, to prove Conjecture 1.1 for $\left(\mathcal{C}_{\sigma}^{\left(i, w_{1,2}, \mathbf{w}_{1}, \mathbf{w}_{2}\right)}, C_{\sigma}^{\left(w_{1,2}, \mathbf{w}_{1}, \mathbf{w}_{2}\right)}\right)$ it is enough to prove the conjecture for $\left(\mathcal{C}^{\left(i, w_{1,2}, \mathbf{w}_{1}, \mathbf{w}_{2}\right)}, C^{\left(w_{1,2}, \mathbf{w}_{1}, \mathbf{w}_{2}\right)}\right)$.

The next proposition verifies Conjecture 1.1 for an infinite collection of pairs $(\mathcal{C}, C)$ such that the $C$ cover $\mathscr{L}(X)$ up to measure 0 . Our collection of $(\mathcal{C}, C)$ involves nonabelian stabilisers in a nontrivial way by the fourth bullet point in the proposition. Furthermore, for every $C$ we prove Conjecture 1.1 for multiple different choices of $\mathcal{C}$, thereby illustrating the flexibility of the conjecture in choosing $\mathcal{C}$.

Proposition 10.7. Set $r \in\{3,4\}$ and let $\mathcal{S} \subset \mathcal{S}^{\prime}$ be the collections of measurable subsets of $|\mathscr{L}(\mathcal{X})| \times$ $\mathscr{L}(X)$ given in Definition 10.5. Then

- $\mathcal{S}$ is an infinite set;

- for all $(\mathcal{C}, C) \in \mathcal{S}^{\prime}$, the measurable sets $\mathcal{C}$ and $C$ both have nonzero measure;

$\circ$ the complement of $\bigcup_{(\mathcal{C}, C) \in \mathcal{S}^{\prime}} C$ has measure 0 ;

$\circ$ for all $(\mathcal{C}, C) \in \mathcal{S}$, every $\psi \in \mathcal{C}$ has special point mapping to the point with $\mathrm{SL}_{2}$ stabiliser; 
○ $C=\mathscr{L}(\pi)(\mathcal{C})$ for all $(\mathcal{C}, C) \in \mathcal{S}^{\prime}$; and

○ Conjecture 1.1 holds for all $(\mathcal{C}, C) \in \mathcal{S}^{\prime}-$ that is,

$$
\mu_{\mathcal{X}}(\mathcal{C})=\mu_{\mathcal{X}}\left(\mathcal{C} \cap \mathscr{L}(\pi)^{-1}(C)\right)=\int_{C} \operatorname{sep}_{\pi, \mathcal{C}} \mathrm{d} \mu_{X}^{\mathrm{Gor}}
$$

Remark 10.8. By definition, for every $(\mathcal{C}, C) \in \mathcal{S}$ and $\psi \in \mathcal{C}$ the matrix corresponding to $\psi$ specialises to the 0 matrix. As a result, the special point of $\psi$ maps to the point in $\mathcal{X}$ with $\mathrm{SL}_{2}$ stabiliser, justifying the fourth bullet point of Proposition 10.7.

The remainder of this section is concerned with the proof of Proposition 10.7. To compute $\mu_{X}^{\text {Gor }}\left(C^{\left(w_{1,2}, \mathbf{w}_{1}, \mathbf{w}_{2}\right)}\right)$, it is enough to compute $\mu_{X}^{\text {Gor }}\left(C^{(\mathbf{w})}\right)$, which is done in Proposition 10.9 (the only result in this section where we impose $r \in\{3,4\}$ as opposed to $r \geq 2$ ).

\section{Proposition 10.9.}

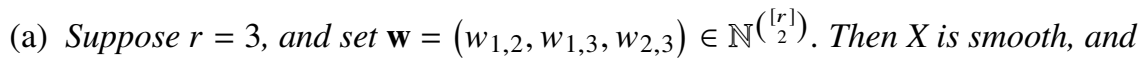

$$
\mu_{X}^{\mathrm{Gor}}\left(C^{(\mathbf{w})}\right)=(\mathbb{L}-1)^{3} \mathbb{L}^{-3-w_{1,2}-w_{1,3}-w_{2,3}} .
$$

(b) Suppose $r=4$, set $\mathbf{w}=\left(w_{1,2}, w_{3,4}, w_{1,3}, w_{2,4}, w_{1,4}, w_{2,3}\right) \in \mathbb{N}^{\left(\begin{array}{c}{[r]} \\ 2\end{array}\right)}$ and set

$$
\begin{aligned}
m & =\min \left(w_{1,2}+w_{3,4}, w_{1,3}+w_{2,4}, w_{1,4}+w_{2,3}\right), \\
j & =-5+m-w_{1,2}-w_{3,4}-w_{1,3}-w_{2,4}-w_{1,4}-w_{2,3} .
\end{aligned}
$$

Then $X$ has log-terminal singularities, and

$$
\mu_{X}^{\mathrm{Gor}}\left(C^{(\mathbf{w})}\right)= \begin{cases}0, & \text { exactly one of } w_{1,2}+w_{3,4}, w_{1,3}+w_{2,4}, w_{1,4}+w_{2,3} \text { equals } m, \\ (\mathbb{L}-1)^{5} \mathbb{L}^{j}, & \text { exactly two of } w_{1,2}+w_{3,4}, w_{1,3}+w_{2,4}, w_{1,4}+w_{2,3} \text { equal } m, \\ (\mathbb{L}-1)^{4}(\mathbb{L}-2) \mathbb{L}^{j}, & \text { all three of } w_{1,2}+w_{3,4}, w_{1,3}+w_{2,4}, w_{1,4}+w_{2,3} \text { equal } m .\end{cases}
$$

Proof.

(a) This is immediate from the fact that $X=\mathbb{A}_{k}^{\left(\begin{array}{c}{[3]} \\ 2\end{array}\right)}$.

(b) We only sketch the proof, as the details are somewhat extensive but do not involve any novel ideas.

We have that $X$ is defined in $\mathbb{A}_{k}^{\left(\begin{array}{c}{[4]} \\ 2\end{array}\right)}$ by the vanishing of a single polynomial $x_{1,2} x_{3,4}-x_{1,3} x_{2,4}+x_{1,4} x_{2,3}$. This polynomial is nondegenerate with respect to its Newton polyhedron, so the claim follows from standard techniques: a certain toric modification of $\mathbb{A}_{k}^{\left(\begin{array}{c}{[4]} \\ 2\end{array}\right)}$ gives a resolution of $X$ (see, for example, [SV09], especially the discrepancy computation in the proof of [SV09, Proposition 2.3]). Note that to get our claim from these techniques, one needs to verify that $\mathrm{e}\left(X \cap \mathbb{G}_{m}^{\left(\begin{array}{c}{[4]} \\ 2\end{array}\right)}\right)=(\mathbb{L}-1)^{3}(\mathbb{L}-2)$, which, for example, follows from the relationship between $X \cap \mathbb{G}_{m}^{\left({ }_{2}{ }^{[4]}\right)}$ and the realisation space of the rank 2 uniform matroid on four elements (see, for example, [Kat16, Proposition 9.7]), which has class $\mathbb{L}-2$.

We next turn to the computation of $\mu_{\mathcal{X}}\left(\mathcal{C}^{\left(i, w_{1,2}, \mathbf{w}_{1}, \mathbf{w}_{2}\right)}\right)$. This is the technical heart of this section.

Proposition 10.10. Let $i, w_{1,2} \in \mathbb{N}$ and $\mathbf{w}_{1}=\left(w_{1,3}, \ldots, w_{1, r}\right), \mathbf{w}_{2}=\left(w_{2,3}, \ldots, w_{2, r}\right)$ satisfy $(\star)$. Then $\mathcal{C}^{\left(i, w_{1,2}, \mathbf{w}_{1}, \mathbf{w}_{2}\right)}$ is a cylinder in $\left|\mathscr{L}_{n}(\mathcal{X})\right|$ and

$$
\mu_{\mathcal{X}}\left(\mathcal{C}^{\left(i, w_{1,2}, \mathbf{w}_{1}, \mathbf{w}_{2}\right)}\right)=(\mathbb{L}-1)^{2 r-3} \mathbb{L}^{-(2 r-3)+(r-4) w_{1,2}-\left(w_{1,3}+\cdots+w_{1, r}+w_{2,3}+\cdots+w_{2, r}\right)} .
$$


Proof. To ease notation, set $Z=Z^{\left(i, w_{1,2}, \mathbf{w}_{1}, \mathbf{w}_{2}\right)}$ and $\mathcal{C}=\mathcal{C}^{\left(i, w_{1,2}, \mathbf{w}_{1}, \mathbf{w}_{2}\right)}$. For any $n \in \mathbb{N}$, let $Z_{n}$ be the locally closed subscheme of $\mathscr{L}_{n}(\widetilde{X})$ whose $A$-valued points, for any $k$-algebra $A$, are the $2 \times r$ matrices of the form

$$
\left(\begin{array}{cccccc}
u_{1,1} t^{w_{1,2}-i} & 0 & u_{1,3} t^{w_{2,3}-i} & u_{1,4} t^{w_{2,4}-i} & \cdots & u_{1, r} t^{w_{2, r}-i} \\
0 & t^{i} & u_{2,3} t^{w_{1,3}-w_{1,2}+i} & u_{2,4} t^{w_{1,4}-w_{1,2}+i} & \cdots & u_{2, r} t^{w_{1, r}-w_{1,2}+i}
\end{array}\right)
$$

where $u_{1,1}, u_{1,3}, \ldots, u_{1, r}, u_{2,3}, \ldots, u_{2, r}$ are all units in $A[t] /\left(t^{n+1}\right)$; so by construction,

$$
Z_{n}=\theta_{n}(Z)
$$

Thus for any $n \in \mathbb{N}$,

$$
\theta_{n}(\mathcal{C})=\mathscr{L}_{n}(\rho)\left(Z_{n}\right)
$$

Now set $n_{0}=\max \left(w_{1,2}, i, w_{2,3}, \ldots, w_{2, r}, w_{1,3}+i, \ldots, w_{1, r}+i\right)$. We will show that

$$
\mathcal{C}=\theta_{n_{0}}^{-1}\left(\mathscr{L}_{n_{0}}(\rho)\left(Z_{n_{0}}\right)\right)
$$

One inclusion is clear from equation (4). To show the other inclusion, let $k^{\prime}$ be a field extension of $k$ and $\psi$ be a $k^{\prime}$-point of $\theta_{n_{0}}^{-1}\left(\mathscr{L}_{n_{0}}(\rho)\left(Z_{n_{0}}\right)\right)$. For the sake of showing the desired inclusion, we may extend $k^{\prime}$, so we may assume there exists a $k^{\prime}$-point $\widetilde{\psi}_{n_{0}}$ of $Z_{n_{0}}$ such that $\mathscr{L}_{n}(\rho)\left(\widetilde{\psi}_{n_{0}}\right) \cong \theta_{n_{0}}(\psi)$. Because $\rho: \widetilde{X} \rightarrow \mathcal{X}$ is smooth, there exists a $k^{\prime}$-point $\tilde{\psi}$ of $\mathscr{L}(\widetilde{X})$ such that $\theta_{n_{0}}(\widetilde{\psi})=\widetilde{\psi}_{n_{0}}$ and $\mathscr{L}(\rho)(\widetilde{\psi}) \cong \psi$. We want to show that there exists some $g \in \mathrm{SL}_{2}\left(k^{\prime} \llbracket t \rrbracket\right)$ such that $g \cdot \widetilde{\psi} \in Z$, as this would imply that $\psi \cong \mathscr{L}(\rho)(\widetilde{\psi}) \cong \mathscr{L}(\rho)(g \cdot \widetilde{\psi}) \in \mathscr{L}(\rho)(Z)=\mathcal{C}$. By our choice of $n_{0}$ and the fact that $\theta_{n_{0}}(\widetilde{\psi}) \in Z_{n_{0}}$, the $\operatorname{arc} \tilde{\psi}$ is equal to

$$
\left(\begin{array}{cccccc}
f_{1,1} & h_{1,2} t^{n_{0}+1} & f_{1,3} & f_{1,4} & \cdots & f_{1, r} \\
h_{2,1} t^{n_{0}+1} & t^{i}+h_{2,2} t^{n_{0}+1} & f_{2,3} & f_{2,4} & \cdots & f_{2, r}
\end{array}\right)
$$

for some $h_{1,2}, h_{2,1}, h_{2,2} \in k^{\prime} \llbracket t \rrbracket$ and some $f_{1,1}, f_{1,3}, \ldots, f_{1, r}, f_{2,3}, \ldots, f_{2, r} \in k^{\prime} \llbracket t \rrbracket$ satisfying

$$
\begin{array}{ll}
\operatorname{ord}_{t}\left(f_{1,1}\right)=w_{1,2}-i, & \\
\operatorname{ord}_{t}\left(f_{1, j}\right)=w_{2, j}-i, & \text { for all } j=3, \ldots, r, \\
\operatorname{ord}_{t}\left(f_{2, j}\right)=w_{1, j}-w_{1,2}+i, & \text { for all } j=3, \ldots, r .
\end{array}
$$

Let $h \in k \llbracket t \rrbracket$ be the $(1,2)$ th minor of $\widetilde{\psi}$. We have $h \neq 0$, because $h$ and $f_{1,1} t^{i}$ have the same image in $k^{\prime}[t] /\left(t^{n_{0}+1}\right)$, and the latter image is nonzero by $n_{0} \geq w_{1,2}$. Thus there exists some $g \in \mathrm{SL}_{2}\left(k^{\prime}(t)\right)$ such that

$$
g \cdot\left(\begin{array}{cc}
f_{1,1} & h_{1,2} t^{n_{0}+1} \\
h_{2,1} t^{n_{0}+1} & t^{i}+h_{2,2} t^{n_{0}+1}
\end{array}\right)=\left(\begin{array}{cc}
h t^{-i} & 0 \\
0 & t^{i}
\end{array}\right)
$$

Then

$$
g^{-1}=\left(\begin{array}{cc}
f_{1,1} & h_{1,2} t^{n_{0}+1} \\
h_{2,1} t^{n_{0}+1} & t^{i}+h_{2,2} t^{n_{0}+1}
\end{array}\right)\left(\begin{array}{cc}
h t^{-i} & 0 \\
0 & t^{i}
\end{array}\right)^{-1}=\left(\begin{array}{cc}
f_{1,1} t^{i} h^{-1} & h_{1,2} t^{n_{0}+1-i} \\
h_{2,1} t^{n_{0}+1+i} h^{-1} & t^{i}+h_{2,2} t^{n_{0}+1-i}
\end{array}\right)
$$


Because $h$ and $f_{1,1} t^{i}$ have the same image in $k^{\prime}[t] /\left(t^{n_{0}+1}\right)$ and $n_{0} \geq w_{1,2}=\operatorname{ord}_{t}\left(f_{1,1} t^{i}\right)$, we have that $\operatorname{ord}_{t}(h)=w_{1,2}$. Together with the fact that $n_{0} \geq i$, this implies that the entries of $g^{-1}$ are all elements of $k^{\prime} \llbracket t \rrbracket$, so $g \in \mathrm{SL}_{2}\left(k^{\prime} \llbracket t \rrbracket\right)$. We will now show that $g \cdot \widetilde{\psi} \in Z$. We see that $g \cdot \widetilde{\psi}$ is equal to

$$
\left(\begin{array}{cccccc}
h t^{-i} & 0 & h_{1,3} & h_{1,4} & \cdots & h_{1, r} \\
0 & t^{i} & h_{2,3} & h_{2,4} & \cdots & h_{2, r}
\end{array}\right)
$$

for some $h_{1,3}, \ldots, h_{1, r}, h_{2,3}, \ldots, h_{2, r} \in k^{\prime} \llbracket t \rrbracket$, and we have already shown that $\operatorname{ord}_{t}\left(h t^{-i}\right)=w_{1,2}-i$. For any $j=3, \ldots, r$, let $q_{1, j} \in k^{\prime} \llbracket t \rrbracket$ (resp., $q_{2, j} \in k^{\prime} \llbracket t \rrbracket$ ) be the $(1, j)$ th (resp., $(2, j)$ th) minor of $\psi$. Then for any $j=3, \ldots, r$, we have that $-f_{1, j} t^{i}$ and $q_{2, j}$ (resp., $f_{1,1} f_{2, j}$ and $q_{1, j}$ ) have the same image in $k^{\prime}[t] /\left(t^{n_{0}+1}\right)$, so because $n_{0} \geq w_{2, j}=\operatorname{ord}_{t}\left(-f_{1, j} t^{i}\right)\left(\operatorname{resp} ., n_{0} \geq w_{1, j}=\operatorname{ord}_{t}\left(f_{1,1} f_{2, j}\right)\right)$, we have $\operatorname{ord}_{t}\left(q_{2, j}\right)=w_{2, j}\left(\operatorname{resp} . \operatorname{ord}_{t}\left(q_{1, j}\right)=w_{1, j}\right)$. Because $g \in \mathrm{SL}_{2}\left(k^{\prime} \llbracket t \rrbracket\right)$, the matrices $\widetilde{\psi}$ and $g \cdot \widetilde{\psi}$ have the same minors, so

$$
\begin{aligned}
& \operatorname{ord}_{t}\left(h_{1, j}\right)=\operatorname{ord}_{t}\left(-q_{2, j} t^{-i}\right)=w_{2, j}-i, \quad \text { for all } j=3, \ldots, r \text {, } \\
& \operatorname{ord}_{t}\left(h_{2, j}\right)=\operatorname{ord}_{t}\left(q_{1, j} h^{-1} t^{i}\right)=w_{1, j}-w_{1,2}+i, \quad \text { for all } j=3, \ldots, r \text {. }
\end{aligned}
$$

We have thus verified that $g \cdot \widetilde{\psi} \in Z$, so $\psi \in \mathcal{C}$, and thus we have finished showing

$$
\mathcal{C}=\theta_{n_{0}}^{-1}\left(\mathscr{L}_{n_{0}}(\rho)\left(Z_{n_{0}}\right)\right)
$$

Because $\mathscr{L}_{n_{0}}(\rho)\left(Z_{n_{0}}\right)$ is a constructible subset of $\mathscr{L}_{n}(\mathcal{X})$ by Chevalley's theorem for Artin stacks [HR17, Theorem 5.2], this implies that $\mathcal{C}$ is a cylinder. We will use the remainder of this proof to compute $\mu_{\mathcal{X}}(\mathcal{C})$.

Set $n_{1}=\max \left(n_{0}, 2 i-1\right)$. For any $n \geq n_{1}$, let $H_{n}$ be the closed subscheme of $\mathscr{L}_{n}\left(\mathbb{A}_{k}^{2 \times 2}\right)$ whose $A$-valued points, for any $k$-algebra $A$, are the $2 \times 2$ matrices of the form

$$
\left(\begin{array}{cc}
1+g_{1,1} t^{n+1-i} & g_{1,2} t^{n+1-i} \\
g_{2,1} t^{n+1-w_{1,2}+i} & 1-g_{1,1} t^{n+1-i}
\end{array}\right)
$$

for some $g_{1,2}, g_{2,1}, g_{2,2} \in A[t] /\left(t^{n+1}\right)$. By our choice of $n_{1}$,

$$
\operatorname{det}\left(\begin{array}{cc}
1+g_{1,1} t^{n+1-i} & g_{1,2} t^{n+1-i} \\
g_{2,1} t^{n+1-w_{1,2}+i} & 1-g_{1,1} t^{n+1-i}
\end{array}\right)=1
$$

and

$$
\begin{aligned}
&\left(\begin{array}{cc}
1+g_{1,1} t^{n+1-i} & g_{1,2} t^{n+1-i} \\
g_{2,1} t^{n+1-w_{1,2}+i} & 1-g_{1,1} t^{n+1-i}
\end{array}\right)\left(\begin{array}{cc}
1+g_{1,1}^{\prime} t^{n+1-i} & g_{1,2}^{\prime} t^{n+1-i} \\
g_{2,1}^{\prime} t^{n+1-w_{1,2}+i} & 1-g_{1,1}^{\prime} t^{n+1-i}
\end{array}\right) \\
&=\left(\begin{array}{cc}
1+\left(g_{1,1}+g_{1,1}^{\prime}\right) t^{n+1-i} & \left(g_{1,2}+g_{1,2}^{\prime}\right) t^{n+1-i} \\
\left(g_{2,1}+g_{2,1}^{\prime}\right) t^{n+1-w_{1,2}+i} & 1-\left(g_{1,1}+g_{1,1}^{\prime}\right) t^{n+1-i}
\end{array}\right)
\end{aligned}
$$

so the inclusion $H_{n} \hookrightarrow \mathscr{L}_{n}\left(\mathbb{A}_{k}^{2 \times 2}\right)$ factors through a closed immersion $H_{n} \hookrightarrow \mathscr{L}_{n}\left(\mathrm{SL}_{2}\right)$ that gives $H_{n}$ the structure of a closed subgroup of $\mathscr{L}_{n}\left(\mathrm{SL}_{2}\right)$, and $H_{n} \cong \mathbb{G}_{a}^{w_{1,2}+i}$ as algebraic groups.

For any $n \in \mathbb{N}$, set $\widetilde{C}_{n}=\mathscr{L}_{n}(\rho)^{-1}\left(\theta_{n}(\mathcal{C})\right) \subset \mathscr{L}_{n}(\widetilde{X})$. We will show that for $n \geq n_{1}$,

$$
\mathrm{e}\left(\widetilde{C}_{n}\right)=\mathrm{e}\left(Z_{n}\right) \mathrm{e}\left(\mathscr{L}_{n}\left(\mathrm{SL}_{2}\right)\right) \mathrm{e}\left(H_{n}\right)^{-1}=\mathrm{e}\left(Z_{n}\right) \mathrm{e}\left(\mathscr{L}_{n}\left(\mathrm{SL}_{2}\right)\right) \mathbb{L}^{-w_{1,2}-i} \in K_{0}\left(\mathbf{S t a c k}_{k}\right)
$$


By equation (4) and Corollary $3.22, \widetilde{C}_{n}$ is equal to the image of the morphism

$$
\mathscr{L}_{n}\left(\mathrm{SL}_{2}\right) \times_{k} Z_{n} \rightarrow \mathscr{L}_{n}(\widetilde{X})
$$

induced by the action of $\mathscr{L}_{n}\left(\mathrm{SL}_{2}\right)$ on $\mathscr{L}_{n}(\widetilde{X})$. Thus to show equation (5), it is sufficient to show that for any field extension $k^{\prime}$ of $k$ and any $\widetilde{\psi}_{n} \in \widetilde{C}_{n}\left(k^{\prime}\right)$, the fibre of $\mathscr{L}_{n}\left(\mathrm{SL}_{2}\right) \times_{k} Z_{n} \rightarrow \mathscr{L}_{n}(\widetilde{X})$ over $\widetilde{\psi}_{n}$ is isomorphic to $H_{n} \otimes_{k} k^{\prime}$. Because $H_{n}$ is special and thus $H_{n}$-torsors over $k^{\prime}$ are trivial, it is sufficient to show that for any field extension $k^{\prime}$ of $k$ and $\widetilde{\psi}_{n} \in Z_{n}\left(k^{\prime}\right)$,

$\circ$ the stabiliser of $\widetilde{\psi}_{n}$ under the $\mathscr{L}_{n}\left(\mathrm{SL}_{2}\right)$ action is $H_{n} \otimes_{k} k^{\prime}$, and

$\circ$ if $g_{n} \in \mathscr{L}_{n}\left(\mathrm{SL}_{2}\right)\left(k^{\prime}\right)$ is such that $g_{n} \cdot \widetilde{\psi}_{n} \in Z_{n}\left(k^{\prime}\right)$, then $g_{n} \cdot \widetilde{\psi}_{n}=\widetilde{\psi}_{n}$.

To show both of these items, it is sufficient to show that for any $g_{n} \in \mathscr{L}_{n}\left(\mathrm{SL}_{2}\right)\left(k^{\prime}\right)$,

$$
g_{n} \cdot \widetilde{\psi}_{n} \in Z_{n}\left(k^{\prime}\right) \Longrightarrow g_{n} \in H_{n}\left(k^{\prime}\right) \Longrightarrow g_{n} \cdot \widetilde{\psi}_{n}=\widetilde{\psi}_{n},
$$

where we note that because $k$ has characteristic 0 , we only need to show that $H_{n} \otimes_{k} k^{\prime}$ and the stabiliser of $\widetilde{\psi}_{n}$ have the same underlying subset of $\mathscr{L}_{n}\left(\mathrm{SL}_{2}\right) \otimes_{k} k^{\prime}$. Write

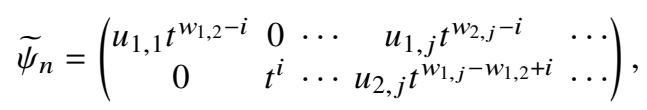

and write

$$
g_{n}=\left(\begin{array}{ll}
a & b \\
c & d
\end{array}\right)
$$

with $a, b, c, d \in k^{\prime}[t] /\left(t^{n+1}\right)$. Then

$$
g_{n} \cdot \widetilde{\psi}_{n}=\left(\begin{array}{lllll}
a u_{1,1} t^{w_{1,2}-i} & b t^{i} & \cdots & a u_{1, j} t^{w_{2, j}-i}+b u_{2, j} t^{w_{1, j}-w_{1,2}+i} & \ldots \\
c u_{1,1} t^{w_{1,2}-i} & d t^{i} & \cdots & c u_{1, j} t^{w_{2, j}-i}+d u_{2, j} t^{w_{1, j}-w_{1,2}+i} & \ldots
\end{array}\right) .
$$

We start with the first implication, so suppose $g_{n} \cdot \widetilde{\psi}_{n} \in Z_{n}\left(k^{\prime}\right)$. Then $b t^{i}=c u_{1,1} t^{w_{1,2}-i}=0$ and $d t^{i}=t^{i}$, so $b=g_{1,2} t^{n+1-i}, c=g_{2,1} t^{n+1-w_{1,2}+i}, d=1-g_{1,1} t^{n+1-i}$ for some units $g_{1,2}, g_{2,1}, g_{1,1}$ in $k^{\prime}[t] /\left(t^{n+1}\right)$. By our choice of $n_{1}$,

$$
1=\operatorname{det} g_{n}=a\left(1-g_{1,1} t^{n+1-i}\right)-g_{1,2} t^{n+1-i} g_{2,1} t^{n+1-w_{1,2}+i}=a\left(1-g_{1,1} t^{n+1-i}\right),
$$

so

$$
a=\left(1-g_{1,1} t^{n+1-i}\right)^{-1}=1+g_{1,1} t^{n+1-i},
$$

and we have $g_{n} \in H_{n}\left(k^{\prime}\right)$. The second implication is a straightforward application of equation (6) and condition $(\star)$. We have therefore finished showing that e $\left(\widetilde{C}_{n}\right)=\mathrm{e}\left(Z_{n}\right) \mathrm{e}\left(\mathscr{L}_{n}\left(\mathrm{SL}_{2}\right)\right) \mathbb{L}^{-w_{1,2}-i}$.

Now we can complete the computation of $\mu_{\mathcal{X}}(\mathcal{C})$. Set

$$
s=w_{1,3}+\cdots+w_{1, r}+w_{2,3}+\cdots+w_{2, r} .
$$

By Corollary 3.22 and the fact that $\mathscr{L}_{n}\left(\mathrm{SL}_{2}\right)$ is a special group, for any $n \geq n_{1}$,

$$
\mathrm{e}\left(\theta_{n}(\mathcal{C})\right)=\mathrm{e}\left(\widetilde{C}_{n}\right) \mathrm{e}\left(\mathscr{L}_{n}\left(\mathrm{SL}_{2}\right)\right)^{-1}=\mathrm{e}\left(Z_{n}\right) \mathbb{L}^{-w_{1,2}-i}=(\mathbb{L}-1)^{2 r-3} \mathbb{L}^{n(2 r-3)+(r-4) w_{1,2}-s}
$$


where the last equality is by the construction of $Z_{n}$. Therefore, noting that $\operatorname{dim} \mathcal{X}=\operatorname{dim}\left(\mathbb{A}_{k}^{2 \times r}\right)-$ $\operatorname{dim} \mathrm{SL}_{2}=2 r-3$,

$$
\mu_{\mathcal{X}}(\mathcal{C})=\lim _{n \rightarrow \infty} \mathrm{e}\left(\theta_{n}(\mathcal{C})\right) \mathbb{L}^{-(n+1) \operatorname{dim} \mathcal{X}}=(\mathbb{L}-1)^{2 r-3} \mathbb{L}^{-(2 r-3)+(r-4) w_{1,2}-s} .
$$

We next compute the value of $\operatorname{sep}_{\pi}$ on our sets of interest.

Proposition 10.11. Let $i, w_{1,2} \in \mathbb{N}$ and $\mathbf{w}_{1}, \mathbf{w}_{2} \in \mathbb{N}^{r-2}$ satisfy ( $\star$. Then the image of $\mathcal{C}^{\left(i, w_{1,2}, \mathbf{w}_{1}, \mathbf{w}_{2}\right)}$

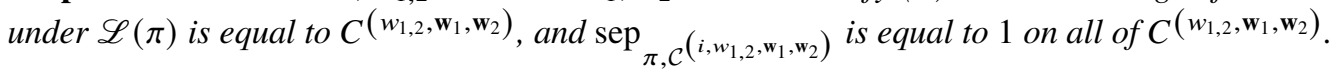

Proof. The image of $\mathcal{C}^{\left(i, w_{1,2}, \mathbf{w}_{1}, \mathbf{w}_{2}\right)}$ is contained in $C^{\left(w_{1,2}, \mathbf{w}_{1}, \mathbf{w}_{2}\right)}$ by the construction of $Z^{\left(i, w_{1,2}, \mathbf{w}_{1}, \mathbf{w}_{2}\right)}$. We now only need to show that sep ${ }_{\pi, \mathcal{C}}^{\left(i, w_{1,2}, w_{1}, w_{2}\right)}$ is equal to 1 on all of $C^{\left(w_{1,2}, w_{1}, w_{2}\right)}$. Let $k^{\prime}$ be a field extension of $k$ and set $\varphi \in C^{\left(w_{1,2}, \mathbf{w}_{1}, \mathbf{w}_{2}\right)}\left(k^{\prime}\right)$. Then for any $\{\ell, m\} \in\left(\begin{array}{c}{[r]} \\ 2\end{array}\right)$, let $h_{\ell, m} \in k^{\prime} \llbracket t \rrbracket$ be such that $\varphi: \operatorname{Spec}\left(k^{\prime} \llbracket t \rrbracket\right) \rightarrow X$ pulls back $\left.x_{\ell, m}\right|_{X}$ to $h_{\ell, m}$. Then $\varphi$ is the image of the element $\widetilde{\psi}$ of $Z^{\left(i, w_{1,2}, \mathbf{w}_{1}, \mathbf{w}_{2}\right)}\left(k^{\prime}\right)$ given by the matrix

$$
\left(\begin{array}{cccccc}
h_{1,2} t^{-i} & 0 & -h_{2,3} t^{-i} & -h_{2,4} t^{-i} & \cdots & -h_{2, r} t^{-i} \\
0 & t^{i} & h_{1,3} h_{1,2}^{-1} t^{i} & h_{1,4} h_{1,2}^{-1} t^{i} & \cdots & h_{1, r} h_{1,2}^{-1} t^{i}
\end{array}\right),
$$

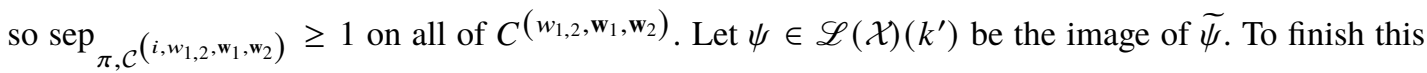
proof, we only need to show that if $\psi^{\prime} \in \mathscr{L}(\mathcal{X})\left(k^{\prime}\right)$ has class in $|\mathscr{L}(\mathcal{X})|$ contained in $\mathcal{C}^{\left(i, w_{1,2}, \mathbf{w}_{1}, \mathbf{w}_{2}\right)}$ and satisfies $\mathscr{L}(\pi)\left(\psi^{\prime}\right)=\varphi$, then $\psi^{\prime} \cong \psi$. Because $\mathrm{SL}_{2}$ is a special group and thus has only trivial torsors over $\operatorname{Spec}\left(k^{\prime} \llbracket t \rrbracket\right)$, there exists $\widetilde{\psi}^{\prime} \in \mathscr{L}(\widetilde{X})\left(k^{\prime}\right)$ whose image is isomorphic to $\psi^{\prime}$. By construction, there exist some field extension $k^{\prime \prime}$ of $k^{\prime}$ and $g \in \mathrm{SL}_{2}\left(k^{\prime \prime} \llbracket t \rrbracket\right)$ such that $g \cdot \widetilde{\psi}_{k^{\prime \prime}}^{\prime} \in Z^{\left(i, w_{1,2}, \mathbf{w}_{1}, \mathbf{w}_{2}\right)}\left(k^{\prime \prime}\right)$, where $\widetilde{\psi}_{k^{\prime \prime}}^{\prime}$ is the composition of $\operatorname{Spec}\left(k^{\prime \prime} \llbracket t \rrbracket\right) \rightarrow \operatorname{Spec}\left(k^{\prime} \llbracket t \rrbracket\right)$ with $\widetilde{\psi}^{\prime}: \operatorname{Spec}\left(k^{\prime} \llbracket t \rrbracket\right) \rightarrow \widetilde{X}$. By the construction of $Z^{\left(i, w_{1,2}, \mathbf{w}_{1}, \mathbf{w}_{2}\right)}$, the entries of $g \cdot \widetilde{\psi}_{k^{\prime \prime}}^{\prime}$ are all determined by its minors and thus $g \cdot \widetilde{\psi}_{k^{\prime \prime}}^{\prime}$ is equal to the composition of $\operatorname{Spec}\left(k^{\prime \prime} \llbracket t \rrbracket\right) \rightarrow \operatorname{Spec}\left(k^{\prime} \llbracket t \rrbracket\right)$ with $\widetilde{\psi}: \operatorname{Spec}\left(k^{\prime} \llbracket t \rrbracket\right) \rightarrow \widetilde{X}$. Because $\tilde{\psi}$ has nonzero $(1,2)$-minor, this implies $g \in \mathrm{SL}_{2}\left(k^{\prime} \llbracket t \rrbracket\right)$, so $g \cdot \widetilde{\psi^{\prime}}=\widetilde{\psi}$ and $\psi^{\prime} \cong \psi$, as desired.

Remark 10.1 and Proposition 10.11 tell us that if $r \geq 3, X$ has log-terminal singularities and $i, w_{1,2} \in \mathbb{N}$ and $\mathbf{w}_{1}, \mathbf{w}_{2} \in \mathbb{N}^{r-2}$ satisfy $(\star)$, then Conjecture 1.1 implies that $\mu_{\mathcal{X}}\left(\mathcal{C}^{\left(i, w_{1,2}, \mathbf{w}_{1}, \mathbf{w}_{2}\right)}\right)=$ $\mu_{X}^{\text {Gor }}\left(C^{\left(w_{1,2}, \mathbf{w}_{1}, \mathbf{w}_{2}\right)}\right)$. For $r \in\{3,4\}$, we verify this unconditionally by combining the foregoing results, thereby proving Proposition 10.7.

Proof of Proposition 10.7. Set $r \in\{3,4\}$. It is clear that $\mathcal{S}$ is an infinite set. The third and fourth bullet points are justified by Remark 10.4 and Remark 10.8. Next, a straightforward computation using Remark 10.4, Proposition 10.9 and Proposition 10.10 shows

$$
0 \neq \mu_{\mathcal{X}}\left(\mathcal{C}^{\left(i, w_{1,2}, \mathbf{w}_{1}, \mathbf{w}_{2}\right)}\right)=\mu_{X}^{\text {Gor }}\left(C^{\left(w_{1,2}, \mathbf{w}_{1}, \mathbf{w}_{2}\right)}\right) .
$$

By Remark 10.6 and Proposition 10.11, this implies

$$
0 \neq \mu_{\mathcal{X}}(\mathcal{C})=\mu_{\mathcal{X}}\left(\mathcal{C} \cap \mathscr{L}(\pi)^{-1}(C)\right)=\int_{C} \operatorname{sep}_{\pi, \mathcal{C}} \mathrm{d} \mu_{X}^{\mathrm{Gor}}
$$

for all $(\mathcal{C}, C) \in \mathcal{S}^{\prime}$

Acknowledgments. We thank Dan Abramovich, Dan Edidin, Jack Hall, Martin Olsson and Karl Schwede for helpful conversations. We are also grateful to the anonymous referee for a thorough reading of our paper and many suggestions which improved the paper. 
The first author's research was partially supported by a Discovery Grant (RGPIN-2015-05631) from the National Science and Engineering Research Council of Canada.

Conflicts of Interest: None.

\section{References}

[Alp13] J. Alper, 'Good moduli spaces for Artin stacks', Ann. Inst. Fourier (Grenoble) 63(6) (2013), 2349-2402.

[Bal08] C. T. Balwe, Geometric Motivic Integration on Artin N-Stacks, Ph.D. thesis, University of Pittsburgh (2008).

[Bal15] C. Balwe, ' $p$-adic and motivic measure on Artin $n$-stacks', Canad. J. Math. 67(6) (2015), 1219-1246.

[Bat98] V. V. Batyrev, 'Stringy Hodge numbers of varieties with Gorenstein canonical singularities', in Integrable Systems and Algebraic Geometry (Kobe/Kyoto, 1997) (World Scientific Publishing, River Edge, NJ, 1998), 1-32.

[Bat99] V. V. Batyrev, 'Non-Archimedean integrals and stringy Euler numbers of log-terminal pairs', J. Eur. Math. Soc. (JEMS) 1(1) (1999), 5-33.

[Bha16] B. Bhatt, 'Algebraization and Tannaka duality', Camb. J. Math. 4(4) (2016), 403-461.

[BM13] V. Batyrev and A. Moreau, 'The arc space of horospherical varieties and motivic integration', Compos. Math. 149(8) (2013), 1327-1352.

[CLNS18] A. Chambert-Loir, J. Nicaise and J. Sebag, Motivic Integration, Progress in Mathematics vol. 325 (Birkhäuser/Springer, New York, 2018).

[CLS11] D. A. Cox, J. B. Little and H. K. Schenck, Toric Varieties, Graduate Studies in Mathematics vol. 124 (American Mathematical Society, Providence, RI, 2011).

[CR04] W. Chen and Y. Ruan, 'A new cohomology theory of orbifold', Comm. Math. Phys. 248(1) (2004), 1-31.

[DG70] M. Demazure and P. Gabriel, Groupes algébriques, Tome I: Géométrie algébrique, généralités, groupes commutatifs (North-Holland Publishing Co., Amsterdam, 1970).

[DL02] J. Denef and F. Loeser, 'Motivic integration, quotient singularities and the McKay correspondence', Compos. Math. 131(3) (2002), 267-290.

[Eke09] T. Ekedahl, 'The Grothendieck group of algebraic stacks', Preprint, 2009, arXiv:0903.3143.

[GS15a] A. Geraschenko and M. Satriano, 'Toric stacks I: The theory of stacky fans', Trans. Amer. Math. Soc. 367(2) (2015), 1033-1071.

[GS15b] A. Geraschenko and M. Satriano, 'Toric stacks II: Intrinsic characterization of toric stacks', Trans. Amer. Math. Soc. 367(2) (2015), 1073-1094.

[HR17] J. Hall and D. Rydh, 'The telescope conjecture for algebraic stacks', J. Topol. 10(3) (2017), 776-794.

[Joy07] D. Joyce, 'Motivic invariants of Artin stacks and "stack functions"', Q. J. Math. 58(3) (2007), 345-392.

[Kat16] E. Katz, 'Matroid theory for algebraic geometers', in Nonarchimedean and Tropical Geometry (Springer, Cham, Switzerland, 2016), 435-517.

[Kon95] M. Kontsevich, 'String cohomology', Lecture at Orsay, December 7, 1995.

[Kre99] A. Kresch, 'Cycle groups for Artin stacks', Invent. Math. 138(3) (1999), 495-536.

[Mil80] J. S. Milne, Étale Cohomology, Princeton Mathematical Series vol. 33 (Princeton University Press, Princeton, NJ, 1980).

[Ols06] M. C. Olsson, 'Deformation theory of representable morphisms of algebraic stacks', Math. Z. 253(1) (2006), $25-62$.

[Rei92] M. Reid, 'The McKay correspondence and the physicists' Euler number', Lecture at University of Utah (Sept.) and MSRI (Nov.), 1992.

[Ryd11] D. Rydh, 'Representability of Hilbert schemes and Hilbert stacks of points', Comm. Algebra 39(7) (2011), $2632-2646$.

[Ryd15] D. Rydh, 'Noetherian approximation of algebraic spaces and stacks', J. Algebra 422(NN) (2015), 105-147.

[Sat13] M. Satriano, 'Canonical Artin stacks over log smooth schemes', Math. Z. 274(3-4) (2013), 779-804.

[Sta21] The Stacks project authors, The Stacks Project (2021). URL: https://stacks.math.columbia.edu.

[SV09] J. Schepers and W. Veys, 'Stringy E-functions of hypersurfaces and of Brieskorn singularities', Adv. Geom. 9(2) (2009), 199-217.

[Ta114] M. Talpo, Infinite Root Stacks of Logarithmic Schemes and Moduli of Parabolic Sheaves, Ph.D. thesis, Scuola Normale Superiore di Pisa (2014).

[Yas04] T. Yasuda, 'Twisted jets, motivic measures and orbifold cohomology', Compos. Math. 140(2) (2004), $396-422$.

[Yas06] T. Yasuda, 'Motivic integration over Deligne-Mumford stacks', Adv. Math. 207(2) (2006), 707-761.

[Yas19] T. Yasuda, 'Motivic integration over wild Deligne-Mumford stacks', Preprint, 2019, arXiv:1908.02932. 